\title{
FRACVAL: Validation (Nonlinear Least Squares Method) of the Solution of One-Dimensional Transport of Decaying Species in a Discrete Planar Fracture With Rock Matrix Diffusion
}

\section{Part 1: Analytical Solutions}

Technical Report

August 1990

\author{
A. Berge Gureghian \\ Office of Waste Technology Development \\ Battelle Energy Systems Group \\ 7000 South Adams Street \\ Willowbrook, IL 60521
}

This report was prepared by Battelle Energy. Systems Croup, Willowbrook, IL, under Contract No. DE-AC02-83CH10139 with the U.S. Departmer of Energy. 


\section{ABSTRACT}

Analytical solutions based on the Laplace transforms are presented for the one-dimensional, transient, advective-dispersive transport of a reacting radionuclide through a discrete planar fracture with constant aperture subject to diffusion in the surrounding rock matrix where both regions of solute migration display residual concentrations. The dispersion-free solutions, which are of closed form, are also reported. The solution assumes that the ground-water flow regime is under steady-state and isothermal conditions and that the rock matrix is homogeneous, isotropic, and saturated with stagnant water. The flow region in both fracture and rock matrix are of the semi-infinite type. The initial conditions include a constant and a spatially varying distribution of solute in the case of the fracture region, and a constant in the case of the rock matrix region. The upstream boundary condition exclusive to the fracture corresponds to an exponentially decaying source of solute, which may be subject to a step or band release mode. The boundary condition corresponding to the rock matrix is implicit and corresponds to the space-time dependent concentration prevailing at the interface between fracture and rock. A zero flux condition is assumed at the downstream end of both fracture and rock.

The verification of the solution was performed by means of related analytical solutions dealing with particular aspects of the transport problem under investigation on the one hand, and a numerical solution capable of handling the complete problem on the other.

The integrals encountered in the general solution are evaluated by means of a composite GaussLegendre quadrature scheme. 
Following the passage of the Nuclear Waste Policy Act of 1982, the Civilian Radioactive Waste Management Program was organized in September 1983. The purpose of this program, managed by the U.S. Department of Energy (DOE), is to develop technology and provide facilities for safe, environmentally acceptable, permanent disposal of high-level waste (HLW). HLW includes wastes from both commercial and defense sources, such as spent (used) fuel from nuclear power reactors, accumulations of wastes from production of nuclear weapons, and solidified wastes from fuel reprocessing.

The information in this report pertains to analytical solutions based on laaploce transforms used in studying radionuclide transport in fractured geologic media for the Repository 'Technology Program. This work was sponsored by the DOE Repository Technology and Transportation Division. 


\section{TABLEOE CONTENTS}

Part 1: Analytical Solutions

Page

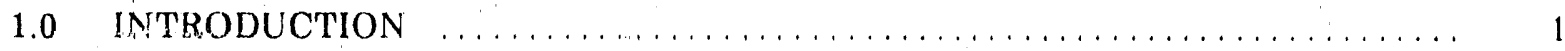

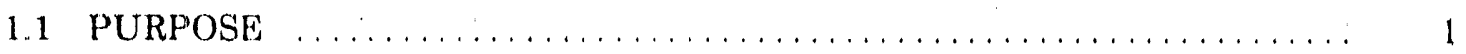

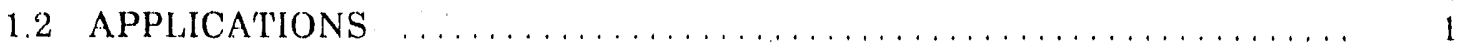

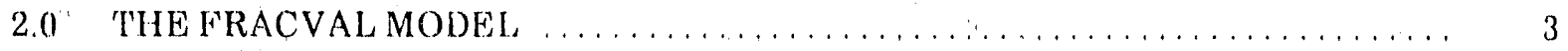

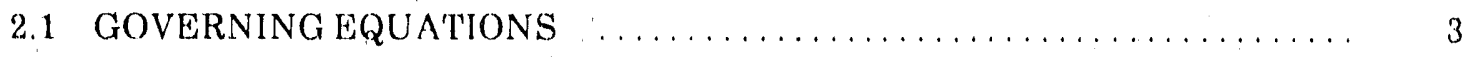

2.1.1 Initial and Boundary Conditions ..................... 5

2.1.2 Solution of Transport Equation for the Rock Matrix and Fracture ..... 6

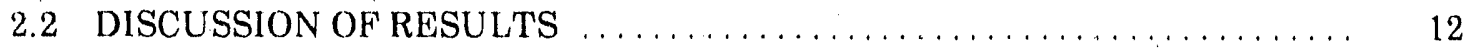

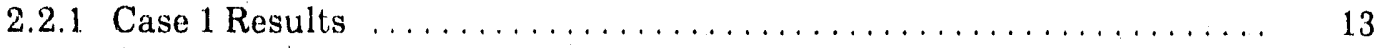

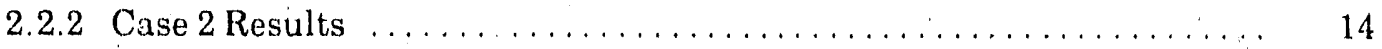

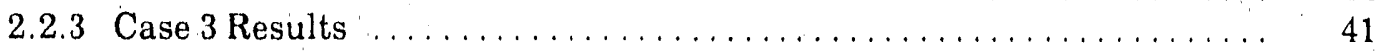

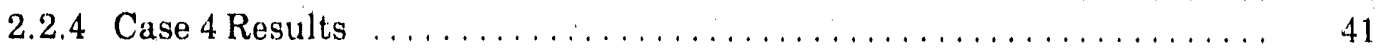

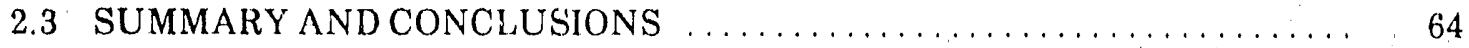

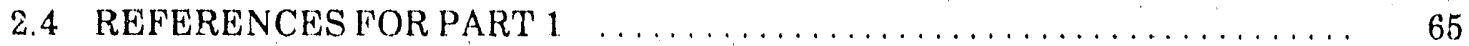

APPENDIX A. THEOREMS AND LAPLACE TRANSFORMS $\ldots \ldots \ldots \ldots \ldots \ldots \ldots$

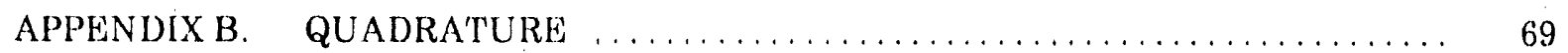

APPENDIX C. EVALUATION OF ERROR FUNCTION AND PRODUCT OF EXPONENTIAL AND COMPLEMENTARY ERROR

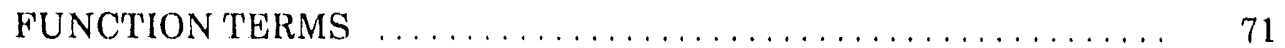

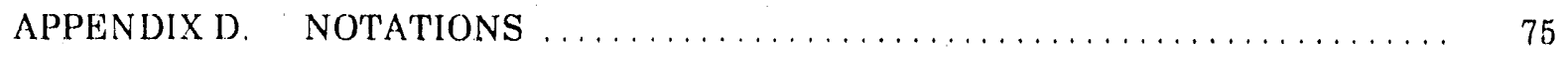

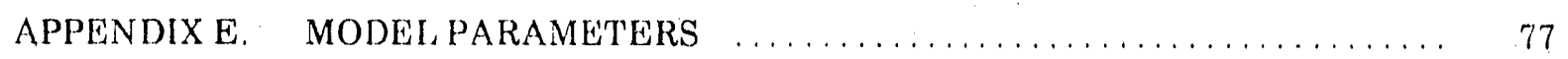

\section{LIST OF' 'TABLES}

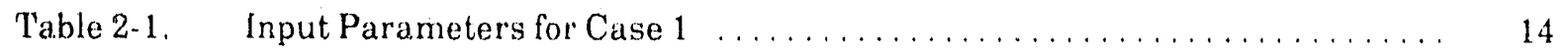

Table 2-2. Case 1A Results: Concentration of Cs-135 at Time $t=3,000$ Years, Zero Matrix Diffusion, Step Release Mode, Retardation Factor

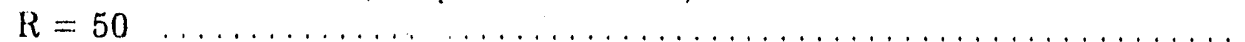

Table 2-3. Case 1A Results: Concentration of Cs-135 at Time $t=3,000$ Years, Zero Matrix Diffusion, Step Release Mode, Retardation Factor $\mathrm{R}=100$ 


\section{LIST OF TABLES}

(Continued)

Page

Table 2-4. Case 1A Results: Concentration of Cs-135 at Time $t=3,000$ Years, Zero Matrix Diffusion, Step Release Mode, Retardation Factor

$R=200$

Table 2-5. Case 1B Results: Concentration of Cs- 135 at Time $t=3,000$ Years,

Zero Matrix Diffusion, Band Release Mode, Retardation Factor

$R=50$

Table 2-6. Case 1B Results: Concentration of Cs-135 at Time $t=3,000$ Years,

Zero Matrix Diffusion, Band Release Mode, Retardation Factor

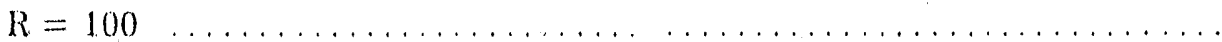

Table 2-7. Case 1B Results: Concentration of Cs-135 at Time $t=3,000$ Years,

Zero Matrix Diffusion, Band Rulease Mode, Retardation Factor

$R=200$

Table 28. Input Parameters for Case 2

Table 2-9. Case 2 Results: Concentration of $\mathrm{Np}-237$ in the Fracture, at

Timet $=10,000$ Years, Step Release Mode, Retardation Factor

$R=1$

Table 2-10. Case 2 Results: Concentration of $\mathrm{Np}-237$ in the Fracture, at

Time $t=10,000$ Years, Step Release Mode, Retardation Factor

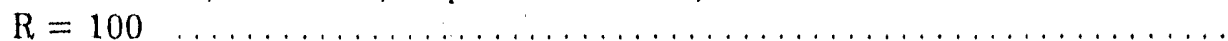

Table 2-11. Case 2 Results: Concentration of Np-237 in the Fracture, at

Time $t=10,000$ Years, Step Release Mode, Retardation Factor

$\mathrm{R}=10,000$

Table 2-12. Case 2 Results: Concentration of Np-237 in the Rock Matrix, at

Time t 10,000 Years, Step Release Mode, Retardation Factor

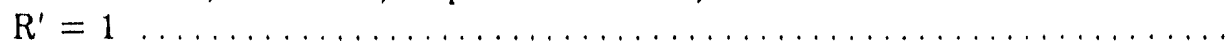

Table 2-13. Case 2 Results: Concentration of $\mathrm{Np}-237$ in the Rock Matrix, at Time $t=10,000$ Years, Step Release Mode, Retardation Factor $\mathrm{R}^{\prime}=100$

Table 2-14. Case 2 Results: Concentration of Np-237 in the Rock Matrix, at Time $t=10,000$ Years, Step Release Mode, Retardation Factor $R^{\prime}=10,000$

Table 2-15. Input Parameters for Case 3

Table 2-16. Case 3 Results: Concentration of $\mathrm{Np}-237$ in the Fracture, at $\mathrm{x}=$ $100 \mathrm{~m}$, Band Release Mode, and Retardation Factor $\mathrm{R}=1$ 


\section{LIST OF'IABLES}

(Continued)

Table 2-17. Case 3 Results: Concentration of $\mathrm{Np}-237$ in the Fracture, at $\mathrm{x}=$ $100 \mathrm{~m}$, Band Release Modo, and Retardation Factor $R=100$

Table 2-18. Case 3 Results: Concentration of $\mathrm{Np}-237$ in the Fracture, at $\mathrm{x}=$ $100 \mathrm{~m}$, Band Release Mode, and Retardation Factor $\mathrm{R}=10,000$

Table 2-19. Case 3 Results: Concentration of $\mathrm{Np}-237$ in the Rock Matrix, at $x=100 \mathrm{~m}$, Time $t=10,000$ Years, Band Release Mode, and

Retardation Factor $\mathrm{R}^{\prime}=1$

Table 2-20. Case 3 Results: Concentration of $\mathrm{Np}-237$ in the Rock Matrix, at $x=100 \mathrm{~m}$, 'Time $t=10,000$ Years, Band Release Mode, and

Retardation Factor $R^{\prime}=100$

Table 2-21. Case 3 Results: Concentration of $\mathrm{Np}-237$ in the Rock Matrix, at $\mathrm{x}=100 \mathrm{~m}$. 'Time $\mathrm{t}=10,000$ Years, Band Release Mode, and

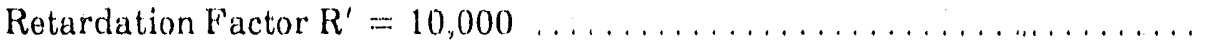

'Table 2-22. Input Parameters for Case 4

Table 2-23. Case 4 Results: Concentration of $\mathrm{Np}-237$ in the Fracture, at

Time $t=5,000$ Years, Band Release Mode, Zero Dispersion,

$\mathrm{D}_{\mathrm{x}}=0 \mathrm{~m}^{2} / \mathrm{yr}$

Table 2-24. Case 4 Results: Concentration of $\mathrm{Np}-237$ in the Fracture, at Time $t=5,000$ Years, Band Release Mode,

$D_{\mathrm{x}}=10 \mathrm{~m}^{2} / \mathrm{y}$

Table 2-25. Case 4 Results: Concentration of $\mathrm{Np}-237$ in the Fracture, at

Time $t=5,000$ Years, Band Release Mode, $D_{x}=100 \mathrm{~m}^{2} / \mathrm{yr} \ldots \ldots \ldots \ldots$

Table 2-26. Case 4 Results: Concentration of $\mathrm{Np}-237$ in the Fracture, at

Time $t=5,000$ Years, Band Release Mode,

$D_{\mathrm{x}}=1,000 \mathrm{~m}^{2} / \mathrm{yr}$

Table 2-27. Case 4 Results: Concentration of $\mathrm{Np}-237$ in the Fracture, at

Time t $=5,000$ Years, Band Release Mode,

$D_{\mathrm{x}}=10,000 \mathrm{~m}^{2} / \mathrm{yr}^{\mathrm{r}}$

'Table 2-28. Case 4 Results: Concentration of Np-237 in the Rock Matrix, at $\mathrm{x}=100 \mathrm{~m}$, 'T'ime $\mathrm{t}=5,000$ Years, Band Release Mode, Zero

Dispersion, $\mathrm{D}=0 \mathrm{~m}^{2} / \mathrm{yl}^{\prime}$

Table 2-29. Case 4 Results: Concentration of $\mathrm{Np-237}$ in the Rock Matrix, at $\mathrm{x}=100 \mathrm{~m}$, T'ime $\mathrm{t}=5,000$ Years, Band Release Mode, Zero

Dispersion, $D=10 \mathrm{~m}^{2} / \mathrm{yr}^{\mathrm{r}}$ 


\section{LIS'T OF TABLES}

(Continued)

$\underline{\text { Page }}$

Table 2-30. Case 4 Results: Concentration of Np-237 in the Rock Matrix, at $\mathrm{x}=100 \mathrm{~m}$, Time $\mathrm{t}=5,000$ Years, Band Release Mode, Zero

Dispersion, $\mathrm{D}=100 \mathrm{~m}^{2} / \mathrm{yr}$

Table 2-31. Case 4 Results: Concentration of Np-237 in the Rock Matrix, at

$\mathrm{x}=100 \mathrm{~m}$, Time $\mathrm{t}=5,000$ Years, Band Release Mode, Zero Dispersion,

$\mathrm{D}=1, \mathrm{G} 00 \mathrm{~m}^{2} / \mathrm{yr}$

60

Table 2-32. Case 4 Results: Concentration of Np-237 in the Rock Matrix, at

$\mathrm{x}=100 \mathrm{~m}$, Time $\mathrm{t}=5,000$ Years, Band Release Mode, Zero Dispersion,

$\mathrm{D}=10,000 \mathrm{~m}^{2} / \mathrm{yr}$ 
Figure 2-1. Relative Concentration of Cs-135 Versus Distance $x$, at 'lime $t=$ 3,000 Years, Zero Matrix Diffusion, for Different Retardation Factor $(R=50,100,200)$ (Case 1A: Step Release Mode)

Figure 2.2. Relative Concentration of Cs-135 Versus Distance $x$, at Timo $t=$ 3,000 Years, Zero Matrix Diffusion, for Different Retardation Factor $(R=50,100,200)$ (Case 1B: Band Release Mode) $\ldots \ldots \ldots \ldots \ldots \ldots$.

Figure 2-3. Relative Concentration of $\mathrm{Np}-23 \%$ Versus Distance $\mathrm{x}$, at Time $\mathrm{t}=$ 10,000 Years, for Different Rock Matrix Retardation Factors $\left(R^{\prime}=1,100,1,000\right)$ (Case 2: Step Release Mode)

Figure 2-4. Relative Concentration of $\mathrm{Np}-237$ Versus Distance $\mathrm{z}$, at Time $\mathrm{t}=$ 10,000 Years, for Different Rock, Matrix Retardation Factors $\left.: R^{\prime}=1,100,10,000\right)$ (Case 2: Step Release Mode)

Figure 2-5. Breakthrough Curves Showing the Relative Concentration of $\mathrm{Np}-237$ in the Fracture Versus Time at $\mathrm{x}=100 \mathrm{~m}$ for Different Rock Matrix Retardation Factors $\left(R^{\prime}=1,100,10,000\right)$

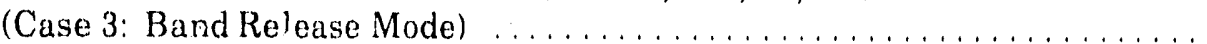

Figure 2-6. Relative Concentration of $\mathrm{Np}-237$ in the Rock Matrix Versus Distance $z$, at Time $t=10,000$ Years, for Different Rock Matrix Retardation Factors $\left(R^{\prime}=1,100,10,000\right)$. (Case 3: Band Release Mode)

Figure 27. Relative Concentration of $\mathrm{Np}-237$ in the Fracture Versus Distance $\mathrm{x}$, at Time $\mathrm{t}=5,000$ Years, for Different Hydrodynamic Dispersion Coefficients $\left(D_{\mathrm{x}}=0,10,100,1,000,10,000 \mathrm{~m}^{2} / \mathrm{yr}\right)$

(Case 4: Band Release Mode)

Figure 2-8. Relative Concentration of $\mathrm{Np}-237$ in the Rock Matrix Versus

Distance $z$, at Time $t=5,000$ Years, for Different Hydrodynamic Dispersion Coefficients $\left(D_{x}=0,10,100,1,000,10,000 \mathrm{~m}^{2 / y r}\right)$

(Case 4: Band Release Mode)

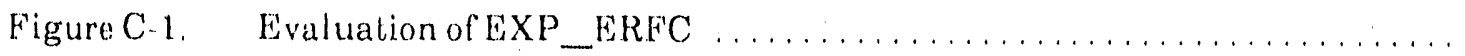




\section{PART 1: ANALYTICAL SOLUTIONS}

\subsection{IN'TRODUCTION}

\subsection{PURPOSE}

'This report is presented in two parts. Part 1 gives the derivation and verification of the analytical solutions based on the Laplace transforms for the one-dimensional isothermal transport of a radionuclide in an idealized discrete planar fracture with constant aperture, including rock matrix diffusion, which accounts for non-zero residual concentrations. Part 2 gives a validation of the analytical solution restricted to the fracture (reported in Part 1) based on the nonlinear least, squares method of uptimization supplemented by a statistical method for analyzing the correlation between estimated parameters and their range of validity.

The verification of the portion of the computer code (i.e., FRACVAL) related to Part 1 is performed by means of available one-dimensional analytical and numerical solutions. The one. dimensional solution of van Genuchten and Alves (1982), which is restricted to a single medium system and which ignores the matrix diffusion process, will be used for benchmarking the performance of this solution. The solution of Gureghian (1990) will be used to verify the performance of the model for a zero residual concentration in both fracture and rock matrix. The verification of the total performance of the model will be carried out by means of a support program ( $\mathrm{S}^{\prime} \mathrm{TEFH}$ ). It is based on the numerica! inversion of the Laplace transforms as proposed by Stefhest (1970); its performance is first estimated by comparison with the benchmarking solutions used in this report.

\subsection{APPLICATIONS}

The solutions presented in this report are primarily related to performance assessment investigations of potential nuclear waste repository sites restricted to typical scenario analyses associated with long-term migration of radionuclides in an idealized fractured rock system. These solutions can be used to simulate the behavior of a decaying species in drill cores in a laboratory environment. Consequently, through a validation process (see Part 2 of this report) they may generate useful sitespecific: information regarding the various parameters gearing the transport process through frac tured rock siystems.

The inodel FRACVAL was written in VAX FOR'TRAN Version 4.8 using the G floating point option (RE $\left.\wedge \mathrm{L}^{*}{ }^{*} 16\right)$. An auxiliary graphies program XYPLO'T (see FRACVAL, User's Guide, Gureghian and Steidl, 1990) was written using DISSPLA Version 10.5. 'The computation was executed on a VAX 8700 under VMS Version 4.7. 


\subsection{THE FRACVAL, MODEL}

The analytical solutions derived in Part 1 of FR $\Lambda C V A L$ are an extension of the ones rolated to the single fracture case reported in Gureghian (1990), and account for a non zoro residual concentration in both fracture and rock matrix. F'or the case of the fracture, the initial conditions may be described by either a constant value or a constant value plus a space-dependent exponential function. However, for'the case of the rock matrix, the initial conditions are restricted to descriptions by a constant value.

To evaluate the semi-infinite solutions related to both fracture and rock matrix, based on the Laplace transforms, an integration must be performed by a composite Gauss-legendre quadrature scheme. When the dispersion mechanism is neglected, closed-form solutions are derived. "The docu. mentation of the analytical solution is given in Soction 2.1 and its verification in Section 2.2. 'The exact derivation of the first-order sensitivities of the concentration in the fracture with respect to the various parameters of the fracture rock system recuired by the various optimization techniques used for the validation of FRACVAL are reported in Part 2 of this report.

\subsection{GOVERNING EQUATIONS}

'The governing one-dimensional equation describing the movement of a typical nuclide in the fracture and rock matrix is given by Gureghian (1990) as follows:

(a) Fracture

$$
R \frac{\partial \Lambda}{\partial t}-D_{x} \frac{\partial^{2} \Lambda}{\partial x^{2}}+u \frac{\partial A}{\partial x}+\lambda R A+\frac{J}{b}=0
$$

(b) Rock Matrix

$$
\left.R^{\prime} \frac{\partial B}{\partial t}-D\right)^{\prime \prime} \frac{\partial^{2} B}{\partial z^{2}}+\lambda R^{\prime} B=0
$$

whore
$A \quad$ is the concentration in the fracturo $\left(\mathrm{ML}_{1} \cdot 3\right)$
$\mathrm{R}$ is the retardation factor in the fracture
$D_{*} \quad$ is the hydrodynamic dispersion coefficient in the $x$ direction of the fracture $\left(l_{2} 2^{\prime} l^{\prime}-1\right)$
$u \quad$ is the average fluid velocity in the fracture (Li' $\left.T^{\prime} \cdot 1\right)$
$\lambda$ is the first order rate constant for docay (' $\mathrm{T}-1$ ) 
$J \quad$ is the diffusive rate of nuclide at surface of fracture per unit area of fracture surface (ML-2T'1)

b is half-thickness of the fracture (L)

$\mathrm{R}^{\prime}$ is the retardation factor in the rock matrix

$B$ Is the concentration in the rock matrix $\left(\mathrm{ML}^{-3}\right)$

$\mathrm{D}_{\mathrm{p}} \quad$ is the pore diffusivity $\left(\mathrm{L}^{2} \mathrm{~T}-1\right)$

$\mathrm{t}$ is the time $(\mathrm{T})$.

The diffusive rate of a nuclide into the rock matrix is assumed to obey Fick's law of diffusion written as

$$
J=-\left.D_{\theta} \frac{\partial B}{\partial z}\right|_{z=b}
$$

where $D_{\theta}$ is the effective diffusivity in the rock matrix (Neretnieks, 1980) defined as

$$
D_{e}=\phi D_{p}
$$

and

$$
D_{p}=D_{d} g_{f}
$$

where

$\phi \quad$ is the rock porosity

$D_{d} \quad$ is the molecular diffusion of nuclide in water ( $\left.L^{2} \mathrm{~T}^{-1}\right)$

$\mathrm{g}_{\mathrm{i}} \quad$ is the geometric factor (i.e., $\delta_{d} / \mathrm{c}^{\prime 2}$ ) where

$\delta_{d} \quad$ is the constrictivity for diffusion $\left(\mathrm{L}^{\circ}\right)$

$\mathrm{l}^{\prime} \quad$ is the tortuosity of the rock matrix $\left(\mathrm{L}^{0}\right)$.

The hydrodynamic dispersion in the fracture (Bear, 1972) is given by

$$
D_{x}=a_{L} u+c D_{d}
$$

where

$a_{L} \quad$ is the longitudinal dispersivity ( $\left.L_{\text {, }}\right)$

$\checkmark \quad$ is the tortuosity of the fracture $\left(L^{(0)}\right)$ 
The retardation factor in the fracture (R) and in tho rock matrix $\left(R^{\prime}\right)$ (Nerotnicks et al., 1982) aro given by:

$$
\begin{gathered}
R=1+K_{r} / b \\
R^{\prime}=1+I(1-\phi) / \phi \mid \rho_{r} K_{r}
\end{gathered}
$$

where

$\rho_{r} \quad$ is the rock density $(M L, i)$

$\mathrm{K}_{\mathrm{f}}$ is the surface distribution coefficient, in the fracture ( $L$ )

$\mathrm{K}_{\mathrm{r}} \quad$ is the distribution coefficient in the rock matrix $\left(\mathrm{L}^{3} \mathrm{M}-1\right)$.

\subsubsection{Initial and Boundary Conditions}

The set of differential equations, Equations 2-1 and 2-2, are subject to the initial conditions:

$$
\begin{gathered}
A(x, 0)=a_{1}+a_{2} e^{-a x}, 0<x<\infty \\
B(x, z, 0)=b_{1}, x>0, \quad z \geq b
\end{gathered}
$$

where

$a_{1}, a_{2}, b_{1}\left(a l l \mathrm{ML}^{-3}\right)$, and $a\left(L^{-1}\right)$ are constant in space and time

and boundary conditions in the fracture and rock matrix. The boundary corditions in the fracture are given by

$$
\begin{gathered}
A(0, t)=A(t) U(t), t>0 \\
\frac{\partial A(\infty, t)}{\partial x}=0, t>0
\end{gathered}
$$

where

$\mathrm{A}(\mathrm{t}) \quad$ is the concentration at the source.

In the rock matrix, the boundary conditions are:

$$
\begin{gathered}
B(x, b, t)=\Lambda(x, t), x>0 \\
\frac{\partial B(x, \infty, t)}{\partial z}=0, t>0
\end{gathered}
$$


For a step release mode (continuous decaving source), the concentration at the source fo: a typical nuclide may be written as

$$
A(t)=A^{0} e^{-\lambda t}
$$

where $\mathrm{A} 0$ is the concentration of the species at time equals zero. For a band release mode, the boundary condition at the inlet may be written as

$$
A(0, t)=A(t)|U(t)-U(t-T)|, t>0
$$

where $\mathrm{T}$ is the leaching time and $\mathrm{U}(\mathrm{t}-\mathrm{T})$ is the Heaviside unit function defined as

$$
\mathrm{U}(\mathrm{t}-\mathrm{T})=\quad \begin{aligned}
& 1, \mathrm{t} \geq \mathrm{T} \\
& 0, \mathrm{t}<\mathrm{T}
\end{aligned}
$$

The general form of the solutions for the band release mode based on a boundary condition given by Equation 2-12 ans. which uses the superposition technique may be written as follows for the fracture and rock matrix:

$$
\begin{aligned}
A(x, t) & =\left[A\left(x, t ; A^{0}, A(x, 0), B(x, z, 0)\right)-A\left(x, t-T ; A^{0} e^{-\lambda T}\right)\right] U(t-T) \\
B(x, z, t) & =\left[B\left(x, z, t ; A^{0}, A(x, 0), B(x, z, 0)\right)-B\left(x, z, t-T ; A^{0} e^{-\lambda T}\right)\right] U(t-T)
\end{aligned}
$$

\subsubsection{Solution of 'I'ransport Equation for the Rock Matrix and F'racture}

\section{Rock Matrix}

The Lapl: transformation of Equation 2-2 with its associated initial and boundary condition Equations 2-7, 2-o, and 2-10 may be written as

$$
\begin{gathered}
D_{p} \frac{d^{2} \bar{B}}{d z^{2}}-R^{\prime}(s+\lambda) \bar{B}=-R^{\prime} b_{1} \\
\bar{B}(x, b, s)=\bar{A}(x, s)
\end{gathered}
$$

where

$$
\bar{B}=\int_{0}^{(x)} B e^{-s t} d t
$$


The general solution of Equation 2-15 is given by

$$
\bar{B}(x, z, s)=\left(\bar{A}-\frac{b_{1}}{s+\lambda}\right) e^{r^{(z-b)}}+\frac{b_{1}}{s+\lambda}
$$

with

$$
r_{b}=-c_{r}(s+\lambda)^{1 / 2}
$$

and

$$
c_{r}=\left(R^{\prime} / D_{p}\right)^{1 / 2}
$$

Note that the inverse Laplace transform of $\bar{B}$ might be sought once $\overline{\mathrm{A}}$ is identified as shown in the subsequent section.

The transformed diffusive flux (Equation 2-3) at the interface of the fracture and rock matrix is given by

$$
\bar{J}=-\phi D_{p} \frac{\partial \bar{B}}{\partial z}(x, b, s)=-\phi D_{p} r_{b}\left(\bar{A}(x, s)-\frac{b_{1}}{s+\lambda}\right) .
$$

Note that $r_{b}$ in the above equation is given by Equation 2-19a.

\section{Fracture}

After proper substitution of the transform of the diffusive flux given by Equation 2-20 into the Laplace transformation of Equation 2-1,

$$
D_{x} \frac{d^{2} \bar{A}}{d x^{2}}-u \frac{\partial \bar{A}}{\partial x}-\left|R(s+\lambda)+c_{f}(s+\lambda)^{1 / 2}\right| \bar{A}=-R\left(a_{1}+a_{2} e^{-d x}\right)-\frac{c_{f} b_{1}}{(s+\lambda)^{1 / 2}}
$$

with

$$
c_{f}=\frac{\Phi}{b}\left(R^{\prime} D_{p}\right)^{1 / 2}
$$

The Laplace transforms of the boundary conditions (Equations 2-9b and 2-11) associated with Equation 2-10 are given by:

$$
\bar{A}(0, s)=\frac{A^{0}}{s+\lambda}
$$




$$
\frac{\partial \overline{\mathrm{A}}}{\partial \mathrm{x}}=0, \mathbf{x}=\infty
$$

The general solution of Equation 2-21 subject to its initial and boundary conditions given by Equations $2-7 \mathrm{~b}$ and $2-9$ may be written as

$$
\bar{A}(x, s)=\left[\frac{A^{0}}{s+1}-R\left[\frac{a_{1}}{r_{a}}+\frac{a_{2}}{r_{a}-p}\right]-\frac{c_{f} b_{1}}{r_{c}}\right] e^{a_{n} x^{2}}+R\left|\frac{a_{1}}{r_{a}}+\frac{a_{2} e^{-a x}}{r_{a}-p}\right|+\frac{c_{f_{1} b_{1}}}{r_{c}}
$$

where

$$
a_{n} x=\frac{1}{2 D_{x}}\left[u-\left(u^{2}+4 r_{a} D_{x}\right)^{1 / 2}\right] x
$$

ard

$$
\begin{gathered}
p=D_{x} a^{2}+u a \\
r_{a}=R(s+\lambda)+c_{f}(s+\lambda)^{1 / 2} \\
r_{c}=r_{a}(s+\lambda)^{1 / 2}
\end{gathered}
$$

Recognizing the following integral (sce Gradshteyn and Ryzhik, 1980)

$$
\int_{0}^{\infty} \exp \left(-\sigma^{2}-\frac{\gamma^{2}}{\sigma^{2}}\right) d \sigma=\frac{\sqrt{\pi}}{2} \exp (-2 \gamma)
$$

Equation 2-24 may now be written in the form

$$
\overline{\mathrm{A}}(\mathrm{x}, \mathrm{s})=\int_{0}^{\infty} \Psi(\mathrm{x}, \sigma)\left(\frac{\mathrm{A}^{0}}{\mathrm{~s}+\lambda}-\mathrm{R}\left[\frac{\mathrm{a}_{1}}{\mathrm{r}_{\mathrm{a}}}+\frac{\mathrm{a}_{2}}{\mathrm{r}_{\mathrm{a}}-\mathrm{p}}\right)-\frac{\mathrm{c}_{\mathrm{f}} \mathrm{b}_{1}}{\mathrm{r}_{\mathrm{c}}}\right) \mathrm{e}^{-\mathrm{r}_{\mathrm{a}} \mathrm{x}} \mathrm{do}+\mathrm{R}\left[\frac{\mathrm{a}_{1}}{\mathrm{r}_{\mathrm{a}}}+\frac{\mathrm{a}_{2} \mathrm{e}^{-\mathrm{ax}}}{\mathrm{r}_{\mathrm{a}}-\mathrm{p}}\right]+\frac{\mathrm{c}_{\mathrm{f}} \mathrm{b}_{1}}{\mathrm{r}_{\mathrm{c}}}
$$

where

$$
\psi(x, \sigma)=\frac{2}{\sqrt{\Pi}} \exp \left|-\left(\sigma-\frac{u x}{4 D_{x} \sigma}\right)^{2}\right|
$$

and

$$
X=\frac{x^{2}}{4 D \sigma^{2}}
$$


Note that ef in Equation 2-26b is given by Equation 2-22. Substitution from Equations 2-26a and 2-26b into Equation 2-28 and using the Laplace inversion formulae (Appendix A), after some manipulation the final solution of the concentration in the fracture may be written as

$$
\begin{aligned}
& \Lambda(x, t)=e^{-\lambda t} \int_{0}^{\infty} \Psi(x, 0)\left\{\left(A^{0}-b_{1}\right) \operatorname{erfc}\left(\frac{c_{f} \cdot X}{2\left(t-R_{X}\right)^{1 / 2}}\right)\right. \\
& -\left(a_{1}-b_{1}\right) \exp \left|\left(\frac{c_{f}}{R}\right)^{2} t\right| \operatorname{erfc}\left|\frac{c_{f}}{R}\left(t-R_{X}\right)^{t / 2}+\frac{c_{f} X}{2\left(t-R_{X}\right)^{1 / 2}}\right| \\
& \left.-\frac{a_{2}}{q} \beta_{2} \exp \left\{\beta_{2} c_{f} X+\beta_{2}^{2}\left(t-R_{X}\right)|\operatorname{erfc}| \beta_{2}\left(t-R_{X}\right)^{1 / 2}+\frac{c_{f} X}{2\left(t-R_{X}\right)^{1 / 2}}\right]\right\} U\left(t-R_{X}\right) d o \\
& +e^{-\lambda t}\left|b_{1}+\left(a_{1}-b_{1}\right) \exp \right|\left(\frac{c_{f}}{R}\right)^{2} t|\operatorname{erfc}| \frac{c_{1}}{R} t^{1 / 2} \mid+\frac{a_{2} e^{-c u x}}{q} \beta_{2} \exp \left(\beta_{2}^{2} t\right) \operatorname{erfc}\left(\beta_{2} t^{1 / 2}\right) \\
& +\frac{a_{2}}{q} \beta_{1} \exp \left[\beta_{1}^{2} t-\lambda t\right]\left|F_{1}(x, t)-F_{2}(x, t)\right| \mid
\end{aligned}
$$

where

$$
\begin{gathered}
F_{1}(x, t)=\int_{0}^{\infty} \psi(x, o) \exp \left|\beta_{1} c_{C} X-\beta_{1}^{2} R_{X}\right| \operatorname{erfc}\left|\beta_{1}\left(t-R_{X}\right)^{1 / 2}+\frac{c_{1} X}{2\left(t-R_{X}\right)^{1 / 2}}\right| U\left(t-R_{X}\right) d o \\
F_{2}(x, t)=\exp (-\alpha x) \operatorname{erfc}\left(\beta_{1} t^{1 / 2}\right)
\end{gathered}
$$

and

$$
q=2\left|\left(\frac{c_{f}}{2 R}\right)^{2}+\frac{p}{R}\right|^{1 / 2}
$$

and

$$
\beta_{1}=\frac{c_{f}}{2 R}-\frac{q}{2}
$$




$$
\beta_{2}=\frac{c_{f}}{2 R}+\frac{q}{2}
$$

Substitution of Equation 2-28 in Equation 2-18 gives

$$
\begin{aligned}
\vec{B}(x, z, s) & =\int_{0}^{\infty} \psi(x, \sigma)\left(\frac{A^{0}}{s+\lambda}-R\left[\frac{a_{1}}{r_{a}}+\frac{a_{2}}{r_{a}-p}\right]-\frac{c_{p} b_{1}}{r_{c}}\right) \exp \left[r_{b}(z-b)-r_{a} X\right] d o \\
& +\left(R\left[\frac{a_{1}}{r_{a}}+\frac{a_{2} e^{-a x}}{r_{a}-p}\right]+\frac{c_{f} b_{1}}{r_{c}}\right) \exp \left[r_{b}(z-b)\right]+\frac{b_{1}}{s+\lambda}\left(1-\exp \left[r_{b}(z-b)\right]\right)
\end{aligned}
$$

and the final solution of the concentration in the rock matrix may be written as

$$
\begin{aligned}
& B(x, z, t)=e^{-\lambda t} \int_{0}^{\infty} \Psi\left(X_{r} \sigma\right)\left\{\left(A^{0}-b_{1}\right)\left|\operatorname{errc}\left(\frac{c_{f} X+c_{r}(z-b)}{2\left(t-R_{X}\right)^{1 / 2}}\right)\right|\right. \\
&\left.-\left(a_{1}-b_{1}\right) \exp \left|\left(c_{f} X+c_{r}(z-b)\right) \frac{c_{f}}{R}+\left(\frac{c_{f}}{R}\right)^{2}(t-R X)\right| \operatorname{erfc} \mid \frac{c_{f}}{R}(t-R X)^{1 / 2}+\frac{c_{f} X+c_{r}(z-b)}{2(t-R X)^{1 / 2}}\right] \\
&\left.\left.-\frac{a_{2}}{q} \beta_{2} \exp \left|\beta_{2}\left(c_{f} X+c_{r}(z-b)\right)+\beta_{2}^{2}(t-R X)\right| \operatorname{erfc} \mid \beta_{2}(t-R X)^{1 / 2}+\frac{c_{f} X+c_{r}(z-b)}{2(t-R X)^{1 / 2}}\right)\right\} U(t-R X) d \sigma \\
&+e^{-\lambda t}\left|b_{1}+\left(a,-b_{1}\right) \exp \right| c_{r}(z-b) \frac{c_{f}}{R}+\left(\frac{c_{f}}{R}\right)^{2} t|\operatorname{erfc}| \frac{c_{f}}{R} t^{1 / 2}+\frac{c_{r}(z-b)}{2 t^{1 / 2}} \mid \\
&+\frac{a_{2}}{q} e^{-a x} \beta_{2} \exp \left|\beta_{2} c_{r}(z-b)+\beta_{2}^{2} t\right| \operatorname{erfc}\left|\beta_{2} t^{1 / 2}+\frac{c_{r}(z-b)}{2 t^{1 / 2}}\right| \\
&+\frac{a_{2}}{q} \beta_{1} \exp \left[\beta_{1}^{2} t-\lambda t\right]\left[G_{1}(x, t)-G_{2}(x, t)\right] \mid
\end{aligned}
$$

where

$$
\begin{aligned}
G_{1}(x, t) & =\int_{0}^{\infty} \Psi(x, 0) \exp \left|\beta_{1}\left(c_{f} X+c_{r}(z-b)\right)-\beta_{1}^{2} R_{X}\right| \operatorname{erfc} \mid \beta_{1}\left(t-R_{X}\right)^{1 / 2} \\
& +\frac{c_{f} X+c_{r}(z-b)}{2\left(t-R_{X}\right)^{1 / 2}} \mid U\left(t-R_{X}\right) d \sigma
\end{aligned}
$$




$$
G_{2}(x, t)=\exp \left|-a x+\beta_{1} c_{1}(z-b)\right| \operatorname{erfc}\left|\beta_{1} t^{1 / 2}+\frac{c_{r}(z-b)}{2 t^{1 / 2}}\right|
$$

Note that $c_{\mathrm{r}}, \mathrm{c}_{\mathrm{f}}, \Psi(\mathrm{x}, \mathrm{0})$, and $\mathrm{x}$ in Equations $2-30$ and $2-34$ are given by Fquations $2-19 \mathrm{~b}, 2-22,2-29 \mathrm{a}$, and $2-296$, respectively.

Alter setting appropriate finite integration limits (see A ppendix B), the integration of Lefua: tions 2-30, 2-30a, 2-34, and 2-34a is then performed using a Gauss-Legondre quadrature scheme.

In the composite quadrature formula adopted in this work, 60 integration points were found to yield a converging quadrature. An exception is made of the integrals given by Equations 2-30a and 2-34a. In the latter case, particularly when the Peclet number (i.e., ux/D) is less than 2 , convergence could be met by subdividing the selected intervals into two more subintervals using 60 integration points each.

\section{Zero Dispersion}

When the hydrodynamic dispersion effects are neglected, Equation 2-21 becomes

$$
u \frac{\partial \bar{A}}{\partial x}+\left|R(s+\lambda)+c_{f}(s+\lambda)^{1 / 2}\right| \bar{A}=R\left(a_{1}+a_{2} e^{-(1 x}\right)+\frac{c_{p} b_{1}}{r_{c}} .
$$

The solution of the above equation subject to its initial and boundary conditions given by Hequations $2-9 b$ and 2-11 may be written as

$$
\bar{A}(x, s)=\left(\frac{A^{0}}{s+\lambda}-R\left(\frac{a_{1}}{r_{a}}+\frac{a_{2}}{r_{a}-p}\right)-\frac{c_{f} b_{1}}{r_{c}}\right) e^{-r_{1} x^{\prime}}+R\left(\frac{a_{1}}{r_{a}}+\frac{a_{2} e^{-a x}}{r_{a}-p}\right)+\frac{c_{f} b_{1}}{r_{c}}
$$

where

$$
x^{\prime}=\frac{x}{u}
$$

The inverse Laplace transforms of $\bar{\AA}(\mathrm{x}, \mathrm{s})$ yielding the solution in the fracture and the rock matrix are given by expressions similar to Equations 2-30 and 2-34 after substitution of $x$ by $x^{\prime}$ and setting

$$
\int_{0}^{\infty} \psi(x, u) d \theta=1
$$


General Case. For large values of the time parameter or small values of tho Peclet number, the $\beta_{1}$, which is a negative constant for a typical problem (seo Equation 2-32a), is a parameter associatod with the nonlinear part of the model describing the residual concontration in the fracturo (sou Equation 2-7). This may tend to cause overflow problems when the components of the solution that include this paramoter are not evaluated indopendontly of the romaining ones. Consequontly, the two componenls of the solution which include parameter $\beta_{1}$ (where one is subject to integration) are combined and represented by the last terms in Equations 2-30 and 2-34, hence made subject to an indepondent evaluation. 'The latter are assumed to correspond to zero when eithor of the following criteria are said to be sutisfied.

$$
\begin{aligned}
& \frac{|F 1-F 2|}{\operatorname{Max}(|F 1|,|F 2|)} \leq 10^{-8} \\
& |F 1-F 2| \leq 10^{-12}
\end{aligned}
$$

In this particular instance, the significant maximum absolute value of the exponential argument used in the computation was restricted to 30 . The implication is that, typically, exponential terms with arguments exceeding this number in absolute value are automatically set to zero.

Zero Dispersion. When dispersion effects are neglected and in viow of Equations 2-37 and 2-38, it may be easily shown that the arguments of the exponential functions in Equations 2-30a and 2-30b are identical. Consequently, when the values of the complementary orror function in both these equations will tend to 2 (i.e., for negative arguments less than or equal to -3), the contribution of the component of the solution in the fracture associated tc parameter $\beta_{1}$ becomas zero. A similar conclusion may be drawn for the solution corresponding to the rock matrix, which will involve in this case Fiquations 2-34a and 2-34b.

The evaluation of the components of the solution presented in this section associated to the product of an exponential and complementary error function is given $\ldots \Lambda_{p p u n d i x} C$.

\subsection{DISCUSSION OF RESULTS}

The analytical solutions presented in this section of the report were vorified by comparison with a vailable one-dimensional closed-form analytical and numerical solutions. 'The one dimensional Gosed form analytical solution reported by van Genuchten and Alves (1982) through their mathemati. 
cal modol EXAC'T', restrictod to a porous medium system, onabled a chock of tho performance of the

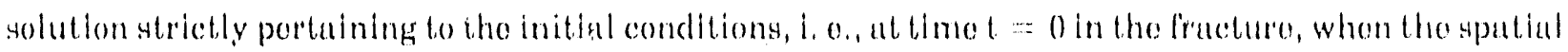
concontration distribution is non uniform (Case 1). In addition, tho solution reforred to tho two typos

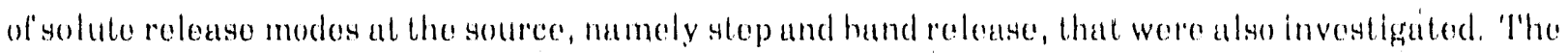
one-dimensional version of the analytical solution of Giureghian (1990) for both fracture and rock matitix was also usod to vorify the solution referred to in the case whoro the residual concentrution in the fracture corresponds to aero and whore the concentration at the souree was subject to a stop rolease: mode (Caso 2). 'The solution scheme proposeri by Stethest (1970) hused on the numerical inversion of' the a aplace transforms whose performance was lirst evaluated in the above montioned lont casers by

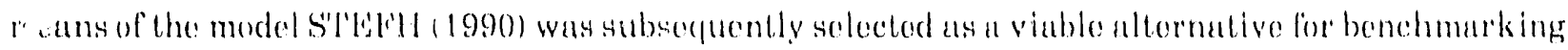

the general form of the new atralytical solution prosented in this section. In this instance, the selected lest (atse (Caso 3) assumes a non-zero residual concentration in both fracture and rock matrix (spatlially varying in the former and constant in the latter loculion). I'he solute roleaso mode is the bund reloaso type. Finally, the general lest case is presented as Cuse 4.

\subsubsection{Cuso 1 Results}

Case 1 examines the concentration of Cy-135 in a ono dimensional domain for a non zero residual concentration distribution in the absence of rock matrix diffusion. In order to compare our results adequately with the solution reported by van Genuchten and Alves (1982), which neglect.4 transfer of solute from the fracture to the rock matrix, the key parameter controlling this particular physical pocess (i. e., the pore diffusivity D) is consecpuently set to zero. Tho solution proposed hy Stefhost (1970), which in this instance operates on the Laplace transforms of Eduation 2-28 with paramoter ef set to zero, was also used in the curront investigation. In the simulations roported here, three values of the rotardation factor wero considered; the type of solute release mode at the source encompasses a step and band release mode. 'The general form of the model simulating the non-zoro residual concentration condition in the fracture (see tequation 22.7) giving way to a non-uniform con. centration distribution at time zero is adopted in these simulations. Based on tho data prosented in T'able 21 , the numorical rosults roported in Tablos 22 through 24 (stop rolouso modo) and 2.5 through 2.7 (band release mode) show excollent agreemont betweon our solution and thoso generaled by the ones reported by both van Genuchten and $\mathrm{Alves}$ (1982) and Stothest (1970). Note that in the latter case 36 integration points were required in ordor to gonorate a stabla and satisfactory solution.

Figures 2-1 and 2-2 show the rolative spat ial distribution of C4. 135 at time corresponding to 3,000 yours for the stop and hand rolease modes and three values of the rotardation factor ( $R$ - :- 50 , 100 , and 200$)$. 
'Table 2-1. Inpul l'arametors for Cane 1

\begin{tabular}{|c|c|}
\hline Species & Cs-135 \\
\hline $\mathrm{T}_{1 / 2}$ & $2.3 \times 10^{6} \mathrm{yr}$ \\
\hline $\begin{array}{l}\text { 'T' (Case 1A: Stop Roloase Mode) } \\
\text { (Case 1B: Band Roloase Mode) }\end{array}$ & $\begin{array}{l}\mathrm{N} / \mathrm{A} \\
10^{3} \mathrm{yr}\end{array}$ \\
\hline $\mathrm{A}^{0 *}$ & 1.0 \\
\hline$a_{1} *$ & 0.1 \\
\hline$a_{2}{ }^{*}$ & $-(0.1)^{* *}$ \\
\hline$a$ & $0.021 / \mathrm{m}$ \\
\hline$D_{p}$ & $0.0 \mathrm{~m}^{2} / \mathrm{yr}$ \\
\hline $\mathrm{u}$ & $2.0 \mathrm{~m} / \mathrm{yr}$ \\
\hline$D_{x}$ & $5.0 \mathrm{~m}^{2} / \mathrm{yr}$ \\
\hline $\mathrm{R}$ & $50,100,200$ \\
\hline
\end{tabular}

*(arbitrary units of activity/L, 3 )

**'This indicates that in Equation 2-7 the constant $+a_{2}$ bocomes $-a_{2}$

\subsubsection{Case 2 Rosults}

Case 2 examines the concentration of $\mathrm{Np}-237$ for a zero rosidual concentration distribution in both fracture and rock including rock matrix diffusion. 'This test case is meant to verify the associated mathematical model and to ostimate the performance of the Stefhest (1970) solution schemo, and to charactorize the conditions when the residual concentration in both fracturo and matrix is assumed to be zero. In the simulations raported hore, throe valuos of the rotardation factor wero considered; the solute injected at the source is subjected to a step release mode. Based on the data presented in T'able 2.8, the numerical results reported in T'ables 2-9) (hrough 2.11 (fracture) and 'T'ables 2.12 through 214 (rock malrix) show that the two solutions F'RACVAl, and S'F'F'll are in excellont agreement with those generatod by I'RACFl, (seo Coroghian, 1990).

Figures 2.3 and 2.4 show the relative spatial distribution of $\mathrm{Np} \cdot 237$ in both fracture and rock matrix at lime corresponding to 10,000 years for the three values of the rock retardation factor $\left(R^{\prime}=1\right.$, $10(0$, and 10,000$)$. 
Table 2.2. Case 1A Results: Concentration of Cs-135 at Time $t=3,000$ Years, Zero Matrix Diffusion, Step

Release Mode, Retardation Factor $\mathrm{R}=50$

\begin{tabular}{cccc}
\hline $\begin{array}{c}\text { Distance } \\
\mathrm{x}(\mathrm{m})\end{array}$ & FRACVAL & EXACT* & STEFH** \\
\hline $0.000 \mathrm{E}+00$ & $9.991 \mathrm{E}-01$ & $9.991 \mathrm{E}-01$ & $9.991 \mathrm{E}-01$ \\
$1.000 \mathrm{E}+01$ & $9.991 \mathrm{E}-01$ & $9.991 \mathrm{E}-01$ & $9.991 \mathrm{E}-0.1$ \\
$2.000 \mathrm{E}+01$ & $9.991 \mathrm{E}-01$ & $9.991 \mathrm{E}-01$ & $9.991 \mathrm{E}-01$ \\
$3.000 \mathrm{E}+01$ & $9.991 \mathrm{E}-01$ & $9.991 \mathrm{E}-01$ & $9.991 \mathrm{E}-01$ \\
$4.000 \mathrm{E}+01$ & $9.988 \mathrm{E}-01$ & $9.988 \mathrm{E}-01$ & $9.988 \mathrm{E}-01$ \\
& & & \\
$5.000 \mathrm{E}+01$ & $9.979 \mathrm{E}-01$ & $9.979 \mathrm{E}-01$ & $9.979 \mathrm{E}-01$ \\
$6.000 \mathrm{E}+01$ & $9.947 \mathrm{E}-01$ & $9.947 \mathrm{E}-01$ & $9.947 \mathrm{E}-01$ \\
$7.000 \mathrm{E}+01$ & $0.851 \mathrm{E}-01$ & $9.851 \mathrm{E}-01$ & $9.851 \mathrm{E}-01$ \\
$8.000 \mathrm{E}+01$ & $9.614 \mathrm{E}-01$ & $9.614 \mathrm{E}-01$ & $9.614 \mathrm{E}-01$ \\
$9.000 \mathrm{E}+01$ & $9.126 \mathrm{E}-01$ & $9.126 \mathrm{E}-01$ & $9.126 \mathrm{E}-01$ \\
& & & \\
$1.000 \mathrm{E}+02$ & $8.280 \mathrm{E}-01$ & $8.281 \mathrm{E}-01$ & $8.281 \mathrm{E}-01$ \\
$1.100 \mathrm{E}+02$ & $7.050 \mathrm{E}-01$ & $7.051 \mathrm{E}-01$ & $7.050 \mathrm{E}-01$ \\
$1.200 \mathrm{E}+02$ & $5.544 \mathrm{E}-01$ & $5.545 \mathrm{E}-01$ & $5.543 \mathrm{E}-01$ \\
$1.3(\mathrm{U} 0 \mathrm{E}+02$ & $3.993 \mathrm{E}-01$ & $3.994 \mathrm{E}-01$ & $3.994 \mathrm{E}-01$ \\
$1.400 \mathrm{E}+02$ & $2.653 \mathrm{E}-01$ & $2.653 \mathrm{E}-01$ & $2.655 \mathrm{E}-01$ \\
& & & \\
$1.500 \mathrm{E}+02$ & $1.685 \mathrm{E}-01$ & $1.685 \mathrm{E}-01$ & $1.685 \mathrm{E}-01$ \\
$1.600 \mathrm{E}+02$ & $1.109 \mathrm{E}-01$ & $1.109 \mathrm{E}-01$ & $1.106 \mathrm{E}-01$ \\
$1.700 \mathrm{E}+02$ & $8.365 \mathrm{E}-02$ & $8.374 \mathrm{E}-02$ & $8.343 \mathrm{E}-02$ \\
$1.800 \mathrm{E}+02$ & $7.487 \mathrm{E}-02$ & $7.495 \mathrm{E}-02$ & $7.492 \mathrm{E}-02$ \\
$1.900 \mathrm{E}+02$ & $7.482 \mathrm{E}-02$ & $7.491 \mathrm{E}-02$ & $7.509 \mathrm{E}-02$ \\
& & & \\
$2.000 \mathrm{E}+02$ & $7.787 \mathrm{E}-02$ & $7.796 \mathrm{E}-02$ & $7.808 \mathrm{E}-02$ \\
$2.100 \mathrm{E}+02$ & $8.144 \mathrm{E}-02$ & $8.153 \mathrm{E}-02$ & $8.142 \mathrm{E}-02$ \\
$2.200 \mathrm{E}+02$ & $8.469 \mathrm{E}-02$ & $8.478 \mathrm{E}-02$ & $8.449 \mathrm{E}-02$ \\
$2.300 \mathrm{E}+02$ & $8.743 \mathrm{E}-02$ & $8.752 \mathrm{E}-02$ & $8.723 \mathrm{E}-02$ \\
$2.400 \mathrm{E}+02$ & $8.969 \mathrm{E}-02$ & $8.978 \mathrm{E}-02$ & $8.963 \mathrm{E}-02$ \\
& & & \\
$2.500 \mathrm{E}+02$ & $9.154 \mathrm{E}-02$ & $9.163 \mathrm{E}-02$ & $9.163 \mathrm{E}-02$ \\
$2.600 \mathrm{E}+02$ & $9.306 \mathrm{E}-02$ & $9.315 \mathrm{E}-02$ & $9.321 \mathrm{E}-02$ \\
$2.700 \mathrm{E}+02$ & $9.430 \mathrm{E}-02$ & $9.439 \mathrm{E}-02$ & $9.441 \mathrm{E}-02$ \\
$2.800 \mathrm{E}+02$ & $9.532 \mathrm{E}-02$ & $9.541 \mathrm{E}-02$ & $9.534 \mathrm{E}-02$ \\
$2.900 \mathrm{E}+02$ & $9.615 \mathrm{E}-02$ & $9.624 \mathrm{E}-02$ & $9.610 \mathrm{E}-02$ \\
$3.000 \mathrm{E}+02$ & $9.683 \mathrm{E}-02$ & $9.692 \mathrm{E}-02$ & $9.676 \mathrm{E}-02$ \\
\hline
\end{tabular}

* EXACT is the nume of the mathematical model

(van Genuchten and Alves, 1982)

** Based on Stefhest, 1970) 
Table 2-3, Case 1A Results: Concentration of Cs-135 at Time $t=3,000$ Years, Zero Matrix Diffusion, Step

Release Mode, Retardation Factor $\mathrm{R}=100$

\begin{tabular}{|c|c|c|c|}
\hline $\begin{array}{c}\text { Distance } \\
\mathrm{x}(\mathrm{m})\end{array}$ & FRACVAL & EXACT & STEFH ${ }^{* *}$ \\
\hline $\begin{array}{l}0.000 \mathrm{E}+00 \\
5.000 \mathrm{E}+00 \\
1.000 \mathrm{E}+01 \\
1.500 \mathrm{E}+01 \\
2.000 \mathrm{E}+01\end{array}$ & $\begin{array}{l}9.991 \mathrm{E}-01 \\
9.990 \mathrm{E}-01 \\
9.986 \mathrm{E}-01 \\
9.974 \mathrm{E}-01 \\
9.945 \mathrm{E}-01\end{array}$ & $\begin{array}{l}9.991 E-01 \\
9.990 E-01 \\
9.986 E-01 \\
9.974 E-01 \\
9.945 E-01\end{array}$ & $\begin{array}{l}9.991 \mathrm{E}-0.1 \\
9.990 \mathrm{E}-01 \\
9.986 \mathrm{E}-01 \\
9.974 \mathrm{E}-01 \\
9.945 \mathrm{E}-01\end{array}$ \\
\hline $\begin{array}{l}2.500 E+01 \\
3.000 E+01 \\
3.500 E+01 \\
4.000 E+01 \\
4.500 E+01\end{array}$ & $\begin{array}{l}9.878 E-01 \\
9.745 E-01 \\
9.504 E-01 \\
9.112 E-01 \\
8.530 E-01\end{array}$ & $\begin{array}{l}9.878 E-01 \\
9.745 E-01 \\
9.504 E-01 \\
9.112 E-01 \\
8.530 E-01\end{array}$ & $\begin{array}{l}9.878 E-01 \\
9.745 E-01 \\
9.504 E-01 \\
9.112 E-01 \\
8.530 E-01\end{array}$ \\
\hline $\begin{array}{l}5.000 \mathrm{E}+01 \\
5.500 \mathrm{E}+01 \\
6.000 \mathrm{E}+01 \\
6.500 \mathrm{E}+01 \\
7.000 \mathrm{E}+01\end{array}$ & $\begin{array}{l}7.743 E-01 \\
6.769 E-01 \\
5.670 E-01 \\
4.534 E-01 \\
3.460 E-01\end{array}$ & $\begin{array}{l}7.743 E-01 \\
6.770 E-01 \\
5.6^{6} 7 E-01 \\
4.534 E-01 \\
3.461 E-01\end{array}$ & $\begin{array}{l}7.743 E-01 \\
6.769 E-01 \\
5.670 E-01 \\
4.534 E-01 \\
3.460 E-01\end{array}$ \\
\hline $\begin{array}{l}7.500 E+01 \\
8.000 E+01 \\
8.500 E+01 \\
9.000 E+01 \\
9.500 E+01\end{array}$ & $\begin{array}{l}2.532 E-01 \\
1.801 E-01 \\
1.278 E-01 \\
9.405 E-02 \\
7.487 E-02\end{array}$ & $\begin{array}{l}2.533 E-01 \\
1.802 E-01 \\
1.279 E-01 \\
9.414 E-02 \\
7.496 E-02\end{array}$ & $\begin{array}{l}2.532 E-01 \\
1.801 E-01 \\
1.278 E-01 \\
9.404 E-02 \\
7.486 E-02\end{array}$ \\
\hline $\begin{array}{l}1.000 \mathrm{E}+02 \\
1.050 \mathrm{E}+02 \\
1.100 \mathrm{E}+02 \\
1.150 \mathrm{E}+02 \\
1.200 \mathrm{E}+02\end{array}$ & $\begin{array}{l}6.578 \mathrm{E}-02 \\
6.293 \mathrm{E}-02 \\
6.347 \mathrm{E}-02 \\
6.560 \mathrm{E}-02 \\
6.832 \mathrm{E}-02\end{array}$ & $\begin{array}{l}6.587 E-02 \\
6.302 E-02 \\
6.356 E-02 \\
6.569 E-02 \\
6.841 E-02\end{array}$ & $\begin{array}{l}6.579 \mathrm{E}-02 \\
6.294 \mathrm{E}-02 \\
6.348 \mathrm{E}-02 \\
6.561 \mathrm{E}-02 \\
6.831 \mathrm{E}-02\end{array}$ \\
\hline $\begin{array}{l}1.250 \mathrm{E}+02 \\
1.300 \mathrm{E}+02 \\
1.350 \mathrm{E}+02 \\
1.400 \mathrm{E}+02 \\
1.450 \mathrm{E}+02\end{array}$ & $\begin{array}{l}7.112 \mathrm{E}-02 \\
7.379 \mathrm{E}-02 \\
7.625 \mathrm{E}-02 \\
7.849 \mathrm{D}-02 \\
8.053 \mathrm{E}-02\end{array}$ & $\begin{array}{l}7.121 E-02 \\
7.388 E-02 \\
7.634 E-02 \\
7.858 E-02 \\
8.062 E-02\end{array}$ & $\begin{array}{l}7.110 \mathrm{E}-02 \\
7.377 \mathrm{E}-02 \\
7.625 \mathrm{E}-02 \\
7.850 \mathrm{E}-02 \\
8.054 \mathrm{E}-02\end{array}$ \\
\hline $\begin{array}{l}1.500 \mathrm{E}+02 \\
1.550 \mathrm{E}+02 \\
1.600 \mathrm{E}+02 \\
1.650 \mathrm{E}+02 \\
1.700 \mathrm{E}+02\end{array}$ & $\begin{array}{l}8.237 E-02 \\
8.404 E-02 \\
8.555 E-02 \\
8.692 E-02 \\
8.815 E-02\end{array}$ & $\begin{array}{l}8.246 E-02 \\
8.413 E-02 \\
8.564 E-02 \\
8.701 E-02 \\
8.82 .5 E-02\end{array}$ & $\begin{array}{l}8.238 \mathrm{E}-02 \\
8.405 \mathrm{E}-02 \\
8.555 \mathrm{E}-02 \\
8.691 \mathrm{E}-02 \\
8.815 \mathrm{E}-02\end{array}$ \\
\hline
\end{tabular}


T'able 2-3, Continued.

\begin{tabular}{cccc}
\hline \hline $\begin{array}{c}\text { Distance } \\
\mathrm{X}(\mathrm{m})\end{array}$ & FRACVAL & EXACT & STEFH** \\
\hline $1.750 \mathrm{E}+02$ & $8.927 \mathrm{E}-02$ & $8.936 \mathrm{E}-02$ & $8.927 \mathrm{E}-02$ \\
$1.800 \mathrm{E}+02$ & $9.029 \mathrm{E}-02$ & $9.038 \mathrm{E}-02$ & $9.028 \mathrm{E}-02$ \\
$1.850 \mathrm{E}+02$ & $9.120 \mathrm{E}-02$ & $9.129 \mathrm{E}-02$ & $9.120 \mathrm{E}-02$ \\
$1.900 \mathrm{E}+02$ & $9.203 \mathrm{E}-02$ & $9.212 \mathrm{E}-02$ & $9.203 \mathrm{E}-02$ \\
$1.950 \mathrm{E}+02$ & $9.278 \mathrm{E}-02$ & $9.287 \mathrm{E}-02$ & $9.278 \mathrm{E}-02$ \\
& & & \\
$2.000 \mathrm{E}+02$ & $9.346 \mathrm{E}-02$ & $9.355 \mathrm{E}-02$ & $9.346 \mathrm{E}-02$ \\
$2.050 \mathrm{E}+02$ & $9.407 \mathrm{E}-02$ & $9.416 \mathrm{E}-02$ & $9.407 \mathrm{E}-02$ \\
$2.100 \mathrm{E}+02$ & $9.463 \mathrm{E}-02$ & $9.472 \mathrm{E}-02$ & $9.463 \mathrm{E}-02$ \\
$2.150 \mathrm{E}+02$ & $9.513 \mathrm{E}-02$ & $9.522 \mathrm{E}-02$ & $9.513 \mathrm{E}-02$ \\
$2.200 \mathrm{E}+02$ & $9.550 \mathrm{E}-02$ & $9.568 \mathrm{E}-02$ & $9.558 \mathrm{E}-02$ \\
& & & \\
$2.250 \mathrm{E}+02$ & $9.600 \mathrm{E}-02$ & $9.609 \mathrm{E}-02$ & $9.600 \mathrm{E}-02$ \\
$2.300 \mathrm{E}+02$ & $9.637 \mathrm{E}-02$ & $9.646 \mathrm{E}-02$ & $9.637 \mathrm{E}-02$ \\
$2.350 \mathrm{E}+02$ & $9.671 \mathrm{E}-02$ & $9.680 \mathrm{E}-02$ & $9.671 \mathrm{E}-02$ \\
$2.400 \mathrm{E}+02$ & $9.701 \mathrm{E}-02$ & $9.710 \mathrm{E}-02$ & $9.701 \mathrm{E}-02$ \\
$2.450 \mathrm{E}+02$ & $9.729 \mathrm{E}-02$ & $9.738 \mathrm{E}-02$ & $9.729 \mathrm{E}-02$ \\
$2.500 \mathrm{E}+02$ & $9.754 \mathrm{E}-02$ & $9.763 \mathrm{E}-02$ & $9.754 \mathrm{E}-02$ \\
$2.550 \mathrm{E}+02$ & $9.776 \mathrm{E}-02$ & $9.785 \mathrm{E}-02$ & $9.776 \mathrm{E}-02$ \\
$2.600 \mathrm{E}+02$ & $9.797 \mathrm{E}-02$ & $9.806 \mathrm{E}-02$. & $9.797 \mathrm{E}-02$ \\
$2.650 \mathrm{E}+02$ & $9.815 \mathrm{E}-02$ & $9.824 \mathrm{E}-02$ & $9.815 \mathrm{E}-02$ \\
$2.700 \mathrm{E}+02$ & $9.832 \mathrm{E}-02$ & $9.841 \mathrm{E}-02$ & $9.832 \mathrm{E}-02$ \\
& & & \\
$2.750 \mathrm{E}+02$ & $9.847 \mathrm{E}-02$ & $9.856 \mathrm{E}-02$ & $9.847 \mathrm{E}-02$ \\
$2.800 \mathrm{E}+02$ & $9.861 \mathrm{E}-02$ & $9.870 \mathrm{E}-02$ & $9.861 \mathrm{E}-02$ \\
$2.850 \mathrm{E}+02$ & $9.873 \mathrm{E}-02$ & $9.882 \mathrm{E}-02$ & $9.873 \mathrm{E}-02$ \\
$2.900 \mathrm{E}+02$ & $9.884 \mathrm{E}-02$ & $9.893 \mathrm{E}-02$ & $9.884 \mathrm{E}-02$ \\
$2.950 \mathrm{E}+02$ & $9.894 \mathrm{E}-02$ & $9.904 \mathrm{E}-02$ & $9.894 \mathrm{E}-02$ \\
& & & \\
$3.000 \mathrm{E}+02$ & $9.904 \mathrm{E}-02$ & $9.913 \mathrm{E}-02$ & $9.904 \mathrm{E}-02$ \\
\hline
\end{tabular}

* EXACT is the name of the mathematical model

(van Genuchten and Alves, 1982)

** Based on Stefhest, 1970 
Table 2-4. Case 1A Results: Concentration of Cs-135 at Time $t=3,000$ Years, Zero Matrix Diffusion, Step

Release Mode, Retardation Factor $R=200$

\begin{tabular}{|c|c|c|c|}
\hline $\begin{array}{c}\text { Distance } \\
\mathrm{x}(\mathrm{m})\end{array}$ & FRACVAL & EXACT" & STEFH** \\
\hline $\begin{array}{l}0.000 \mathrm{E}+00 \\
3.000 \mathrm{E}+00 \\
6.000 \mathrm{E}+00 \\
9.000 \mathrm{E}+00 \\
1.200 \mathrm{E}+01\end{array}$ & $\begin{array}{l}9.991 \mathrm{E}-01 \\
9.971 \mathrm{E}-01 \\
9.923 \mathrm{E}-01 \\
9.827 \mathrm{E}-01 \\
9.656 \mathrm{E}-01\end{array}$ & $\begin{array}{l}9.991 \mathrm{E}-01 \\
9.971 \mathrm{E}-01 \\
9.923 \mathrm{E}-01 \\
9.827 \mathrm{E}-01 \\
9.656 \mathrm{E}-01\end{array}$ & $\begin{array}{l}9.991 \mathrm{E}-01 \\
9.971 \mathrm{E}-01 \\
9.923 \mathrm{E}-01 \\
9.827 \mathrm{E}-01 \\
9.656 \mathrm{E}-01\end{array}$ \\
\hline $\begin{array}{l}1.500 \mathrm{E}+01 \\
1.800 \mathrm{E}+01 \\
2.100 \mathrm{E}+01 \\
2.400 \mathrm{E}+01 \\
2.700 \mathrm{E}+01\end{array}$ & $\begin{array}{l}9.379 \mathrm{E}-01 \\
8.967 \mathrm{E}-01 \\
8.401 \mathrm{E},-01 \\
7.678 \mathrm{E}-01 \\
6.817 \mathrm{E}-01\end{array}$ & $\begin{array}{l}9.379 \mathrm{E}-01 \\
8.967 \mathrm{E}-01 \\
8.402 \mathrm{E}-01 \\
7.678 \mathrm{E}-01 \\
6.818 \mathrm{E}-01\end{array}$ & $\begin{array}{l}9.379 E-01 \\
8.967 E-01 \\
8.401 E-01 \\
7.678 E-01 \\
6.817 E-01\end{array}$ \\
\hline $\begin{array}{l}3.000 \mathrm{E}+01 \\
3.3 \mathrm{WUE}+01 \\
3.600 \mathrm{E}+01 \\
3.900 \mathrm{E}+01 \\
4.200 \mathrm{E}+01\end{array}$ & $\begin{array}{l}5.860 E-01 \\
4.863 E-01 \\
3.893 E-01 \\
3.009 E-01 \\
2.254 E-01\end{array}$ & $\begin{array}{l}5.860 \mathrm{E}-01 \\
4.864 \mathrm{E}-01 \\
3.894 \mathrm{E}-01 \\
3.010 \mathrm{E}-01 \\
2.255 \mathrm{E}-01\end{array}$ & $\begin{array}{l}5.860 E-01 \\
4.863 E-01 \\
3.893 E-01 \\
3.009 E-01 \\
2.254 E-01\end{array}$ \\
\hline $\begin{array}{l}4.500 \mathrm{E}+01 \\
4.800 \mathrm{E}+01 \\
5.100 \mathrm{E}+01 \\
5.400 \mathrm{E}+01 \\
5.700 \mathrm{E}+01\end{array}$ & $\begin{array}{l}1.652 E-01 \\
1.204 E-01 \\
8.942 E-02 \\
6.978 E-02 \\
5.860 E-02\end{array}$ & $\begin{array}{l}1.653 \mathrm{E}-01 \\
1.205 \mathrm{E}-01 \\
8.950 \mathrm{E}-02 \\
6.986 \mathrm{E}-02 \\
5.869 \mathrm{E}-02\end{array}$ & $\begin{array}{l}1.652 E-01 \\
1.204 E-01 \\
8.942 E-02 \\
6.978 E-02 \\
5.860 E-02\end{array}$ \\
\hline $\begin{array}{l}6.000 \mathrm{E}+01 \\
6.300 \mathrm{E}+01 \\
6.600 \mathrm{E}+01 \\
6.900 \mathrm{E}+01 \\
7.200 \mathrm{E}+01\end{array}$ & $\begin{array}{l}5.326 E-02 \\
5.161 E-02 \\
5.212 E-02 \\
5.375 E-02 \\
5.590 E-02\end{array}$ & $\begin{array}{l}5.335 \mathrm{E}-02 \\
5.170 \mathrm{E}-02 \\
5.221 \mathrm{E}-02 \\
5.384 \mathrm{E}-02 \\
5.599 \mathrm{E}-02\end{array}$ & $\begin{array}{l}5.326 \mathrm{E}-02 \\
5.161 \mathrm{E}-02 \\
5.212 \mathrm{E}-02 \\
5.375 \mathrm{E}-02 \\
5.590 \mathrm{E}-02\end{array}$ \\
\hline $\begin{array}{l}7.500 \mathrm{E}+01 \\
7.800 \mathrm{E}+01 \\
8.100 \mathrm{E}+01 \\
8.400 \mathrm{E}+01 \\
8.700 \mathrm{E}+01\end{array}$ & $\begin{array}{l}5.823 \mathrm{E}-02 \\
6.056 \mathrm{E}-02 \\
6.281 \mathrm{E}-02 \\
6.496 \mathrm{E}-02 \\
6.699 \mathrm{E}-02\end{array}$ & $\begin{array}{l}5.832 E-02 \\
6.065 E-02 \\
6.290 E-02 \\
6.505 E-02 \\
6.708 E-02\end{array}$ & $\begin{array}{l}5.823 \mathrm{E}-02 \\
6.055 \mathrm{E}-02 \\
6.281 \mathrm{E}-02 \\
6.496 \mathrm{E}-02 \\
6.699 \mathrm{E}-02\end{array}$ \\
\hline $\begin{array}{l}9.000 \mathrm{E}+01 \\
9.300 \mathrm{E}+01 \\
9.600 \mathrm{E}+01 \\
9.900 \mathrm{E}+01 \\
1.020 \mathrm{E}+02\end{array}$ & $\begin{array}{l}6.890 \mathrm{E}-02 \\
7.071 \mathrm{E}-02 \\
7.241 \mathrm{E}-02 \\
7.401 \mathrm{E}-02 \\
7.552 \mathrm{E}-02\end{array}$ & $\begin{array}{l}6.899 \mathrm{E}-02 \\
7.080 \mathrm{E}-02 \\
7.250 \mathrm{E}-02 \\
7.410 \mathrm{E}-02 \\
7.561 \mathrm{E}-02\end{array}$ & $\begin{array}{l}6.890 \mathrm{E}-02 \\
7.071 \mathrm{E}-02 \\
7.241 \mathrm{E}-02 \\
7.401 \mathrm{E}-02 \\
7.552 \mathrm{E}-02\end{array}$ \\
\hline
\end{tabular}


Table 2.4, Continued.

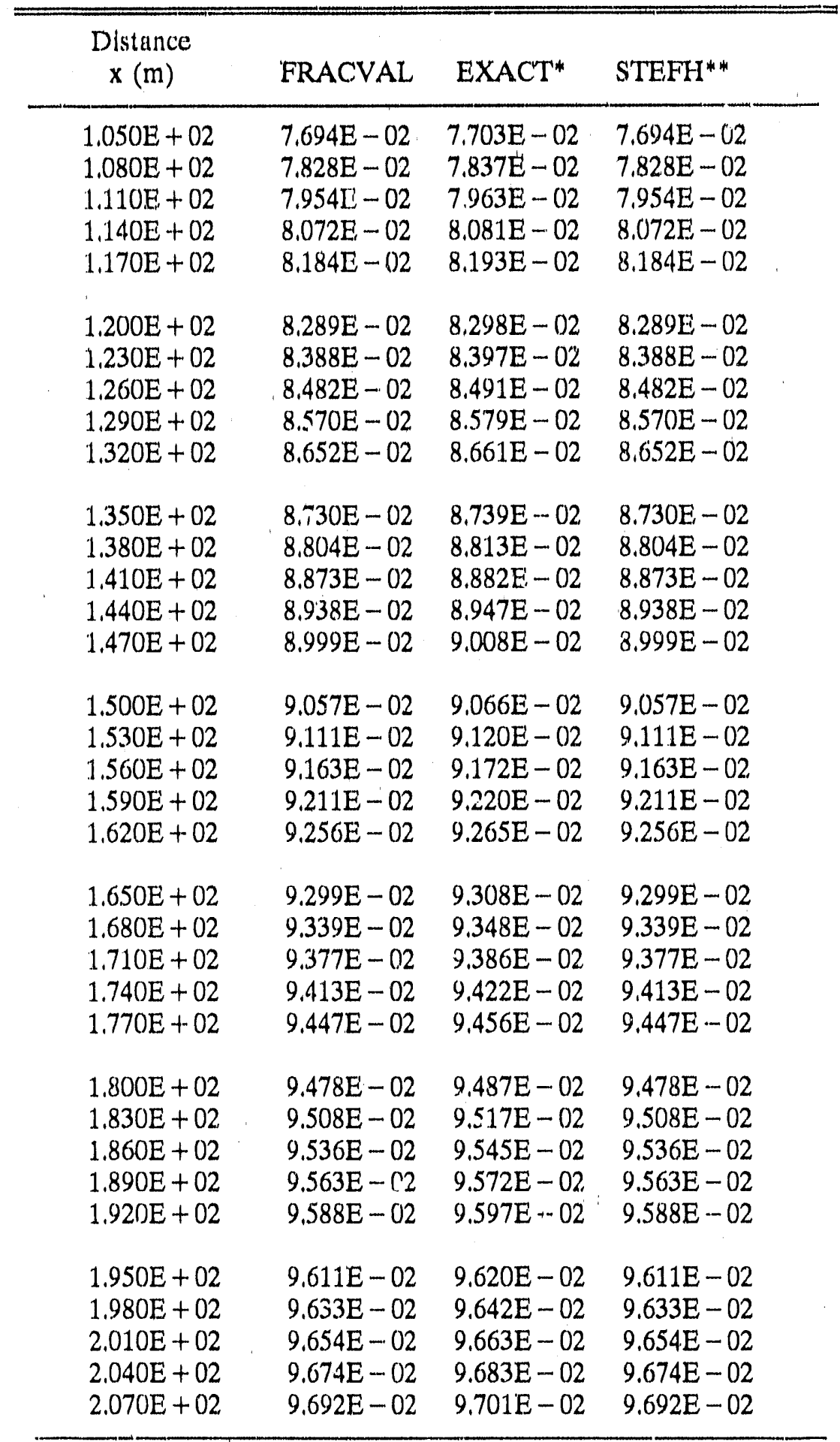


Table 2-4. Continued.

\begin{tabular}{cccl}
\hline $\begin{array}{c}\text { Distance } \\
\text { X }(\mathrm{m})\end{array}$ & FRACVAL & EXACT* & STEFH ${ }^{* *}$ \\
\hline $2.100 \mathrm{E}+02$ & $9.710 \mathrm{E}-02$ & $9.719 \mathrm{E}-02$ & $9.710 \mathrm{E}-02$ \\
$2.130 \mathrm{E}+02$ & $9.726 \mathrm{E}-02$ & $9.735 \mathrm{E}-02$ & $9.726 \mathrm{E}-02$ \\
$2.160 \mathrm{E}+02$ & $9.741 \mathrm{E}-02$ & $9.751 \mathrm{E}-02$ & $9.741 \mathrm{E}-02$ \\
$2.190 \mathrm{E}+02$ & $9.756 \mathrm{E}-02$ & $9.765 \mathrm{E}-02$ & $9.756 \mathrm{E}-02$ \\
$2.220 \mathrm{E}+02$ & $9.770 \mathrm{E}-02$ & $9.779 \mathrm{E}-02$ & $9.770 \mathrm{E}-02$ \\
& & & \\
$2.250 \mathrm{E}+02$ & $9.783 \mathrm{E}-02$ & $9.792 \mathrm{E}-02$ & $9.783 \mathrm{E}-02$ \\
$2.280 \mathrm{E}+02$ & $9.795 \mathrm{E}-02$ & $9.804 \mathrm{E}-02$ & $9.795 \mathrm{E}-02$ \\
$2.310 \mathrm{E}+02$ & $9.806 \mathrm{E}-02$ & $9.815 \mathrm{E}-02$ & $9.806 \mathrm{E}-02$ \\
$2.340 \mathrm{E}+02$ & $9.817 \mathrm{E}-02$ & $9.826 \mathrm{E}-02$ & $9.817 \mathrm{E}-02$ \\
$2.370 \mathrm{E}+02$ & $9.827 \mathrm{E}-02$ & $9.836 \mathrm{E}-02$ & $9.827 \mathrm{E}-02$ \\
& & & \\
$2.400 \mathrm{E}+02$ & $9.837 \mathrm{E}-02$ & $9.846 \mathrm{E}-02$ & $9.837 \mathrm{E}-02$ \\
$2.430 \mathrm{E}+02$ & $9.846 \mathrm{E}-02$ & $9.855 \mathrm{E}-02$ & $9.846 \mathrm{E}-02$ \\
$2.460 \mathrm{E}+02$ & $9.854 \mathrm{E}-02$ & $9.863 \mathrm{E}-02$ & $9.854 \mathrm{E}-02$ \\
$2.490 \mathrm{E}+02$ & $9.862 \mathrm{E}-02$ & $9.871 \mathrm{E}-02$ & $9.862 \mathrm{E}-02$ \\
$2.520 \mathrm{E}+02$ & $9.870 \mathrm{E}-02$ & $9.879 \mathrm{E}-02$ & $9.870 \mathrm{E}-02$ \\
& & & \\
$2.550 \mathrm{E}+02$ & $9.877 \mathrm{E}-02$ & $9.886 \mathrm{E}-02$ & $9.877 \mathrm{E}-02$ \\
$2.580 \mathrm{E}+02$ & $9.883 \mathrm{E}-02$ & $9.892 \mathrm{E}-02$ & $9.883 \mathrm{E}-02$ \\
$2.610 \mathrm{E}+02$ & $9.890 \mathrm{E}-02$ & $9.899 \mathrm{E}-02$ & $9.890 \mathrm{E}-02$ \\
$2.640 \mathrm{E}+02$ & $9.895 \mathrm{E}-02$ & $9.904 \mathrm{E}-02$ & $9.895 \mathrm{E}-02$ \\
$2.670 \mathrm{E}+02$ & $9.901 \mathrm{E}-02$ & $9.910 \mathrm{E}-02$ & $9.901 \mathrm{E}-02$ \\
& & & \\
$2.700 \mathrm{E}+02$ & $9.906 \mathrm{E}-02$ & $9.915 \mathrm{E}-02$ & $9.906 \mathrm{E}-02$ \\
$2.730 \mathrm{E}+02$ & $9.911 \mathrm{E}-02$ & $9.920 \mathrm{E}-02$ & $9.911 \mathrm{E}-02$ \\
$2.760 \mathrm{E}+02$ & $9.916 \mathrm{E}-02$ & $9.925 \mathrm{E}-02$ & $9.916 \mathrm{E}-02$ \\
$2.790 \mathrm{E}+02$ & $9.920 \mathrm{E}-02$ & $9.929 \mathrm{E}-02$ & $9.920 \mathrm{E}-02$ \\
$2.820 \mathrm{E}+02$ & $9.924 \mathrm{E}-02$ & $9.933 \mathrm{E}-02$ & $9.924 \mathrm{E}-02$ \\
& & & \\
$2.850 \mathrm{E}+02$ & $9.928 \mathrm{E}-02$ & $9.937 \mathrm{E}-02$ & $9.928 \mathrm{E}-02$ \\
$2.880 \mathrm{E}+02$ & $9.932 \mathrm{E}-02$ & $9.941 \mathrm{E}-02$ & $9.932 \mathrm{E}-02$ \\
$2.910 \mathrm{E}+02$ & $9.935 \mathrm{E}-02$ & $9.944 \mathrm{E}-02$ & $9.935 \mathrm{E}-02$ \\
$2.940 \mathrm{E}+02$ & $9.939 \mathrm{E}-02$ & $9.948 \mathrm{E}-02$ & $9.939 \mathrm{E}-02$ \\
$2.970 \mathrm{E}+02$ & $9.942 \mathrm{E}-02$ & $9.951 \mathrm{E}-02$ & $9.942 \mathrm{E}-02$ \\
$3.000 \mathrm{E}+02$ & $9.944 \mathrm{E}-02$ & $9.954 \mathrm{E}-02$ & $9.944 \mathrm{E}-02$ \\
\hline \hline
\end{tabular}

* EXACT is the name of the mathematical model (van Genuchten and Alves, 1982)

** Based on Stefhest, 1970 
Table 2-5. Case 1B Results: Concentration of Cs-135 at Time $t=3,000$ Years, Zero Matrix Diffusion, Band

Release Mode, Retardation Factor $\mathrm{R}=50$.

\begin{tabular}{|c|c|c|c|}
\hline $\begin{array}{c}\text { Distance } \\
\times(\pi 1)\end{array}$ & FRACVAL & $\mathrm{EXACT}^{*}$ & STEFH** \\
\hline $1.000 \mathrm{E}+01$ & (i. $251 \mathrm{E}-09$ & $6.251 \mathrm{E}-09$ & $6.274 \mathrm{E}-09$ \\
\hline $2.00 \mathrm{~J} \mathrm{E}+01$ & $7.730 \mathrm{E}-08$ & $7.730 \mathrm{E}-08$ & $7.735 E-08$ \\
\hline $3.000 \mathrm{E}+01$ & $6.368 \mathrm{E}-07$ & $6.368 \mathrm{E}-07$ & $6.214 E-07$ \\
\hline $4.000 \mathrm{E}+01$ & $4.142 E-06$ & $4.142 \mathrm{E}-06$ & $3.949 \mathrm{E}-06$ \\
\hline $5.000 \mathrm{E}+01$ & $2.244 \mathrm{E}-05$ & $2.246 \mathrm{E}-05$ & $2.353 E-05$ \\
\hline $6.000 \mathrm{E}+01$ & $1.038 \mathrm{E}-04$ & $1.038 \mathrm{E}-04$ & $1.066 \mathrm{E}-04$ \\
\hline $7.000 \mathrm{E}+01$ & $4.150 \mathrm{E}-04$ & $4.151 E-04$ & $4.040 E-04$ \\
\hline $8.000 \mathrm{E}+01$ & $1.447 \mathrm{E}-03$ & $1.447 \mathrm{E}-03$ & $1.425 E-03$ \\
\hline $9.000 \mathrm{E}+01$ & $4.425 \mathrm{E}-03$ & $4.425 \mathrm{E}-03$ & $4.461 E-03$ \\
\hline 1.0100 +02 & $1.191 \mathrm{E}-02$ & $1.191 \mathrm{E}-02$ & $1.202 E-02$ \\
\hline $1.100 \mathrm{E}+02$ & $2.836 \mathrm{E}-02$ & $2.836 \mathrm{E}-02$ & $2.835 \mathrm{E}-02$ \\
\hline $1.200 \mathrm{E}+02$ & $5.985 \mathrm{E}-02$ & $5.986 \mathrm{E}-02$ & $5.958 \mathrm{E}-02$ \\
\hline $1.300 E+02$ & $1.124 \mathrm{E}-01$ & $1.124 \mathrm{E}-01$ & $1.121 E-01$ \\
\hline $1.400 E+02$ & $1.882 \mathrm{E}-01$ & $1.882 E-01$ & $1.884 \mathrm{E}-01$ \\
\hline $1.500 \mathrm{E}+02$ & $2.820 \mathrm{E}-01$ & $2.820 \mathrm{E}-01$ & $2.828 \mathrm{E}-01$ \\
\hline $1.600 \mathrm{E}+02$ & $3.795 \mathrm{E}-01$ & $3.795 \mathrm{E}-01$ & $3.801 E-01$ \\
\hline $1.700 \mathrm{E}+02$ & $4.601 \mathrm{E}-01$ & $4.602 E-01$ & $4.598 \mathrm{E}-01$ \\
\hline $1.800 \mathrm{E}+02$ & $5.047 \mathrm{E}-01$ & $5.048 \mathrm{E}-01$ & $5.034 \mathrm{E}-01$ \\
\hline $1.900 \mathrm{E}+02$ & $5.031 \mathrm{E}-01$ & $5.032 E-01$ & $5.017 \mathrm{E}-01$ \\
\hline $2.000 \mathrm{E}+02$ & $4.582 \mathrm{E}-01$ & $4.583 \mathrm{E}-01$ & $4.581 \mathrm{E}-01$ \\
\hline $2.1 \mathrm{COE}+02$ & $3.842 \mathrm{E}-01$ & $3.843 E-01$ & $3.857 \mathrm{E}-01$ \\
\hline $2.200 \mathrm{E}+02$ & $3.001 \mathrm{E}-01$ & $3.002 E-01$ & $3.022 E-01$ \\
\hline $2.300 E+02$ & $2.226 \mathrm{E}-01$ & $2.227 E-01$ & $2.239 E-01$ \\
\hline $2.400 \mathrm{E}+02$ & $1.619 \mathrm{E}-01$ & $1.620 E-01$ & $1.615 \mathrm{E}-01$ \\
\hline $2.500 \mathrm{E}+02$ & $1.209 \mathrm{E}-01$ & $1.210 \mathrm{E}-01$ & $1.190 \mathrm{E}-01$ \\
\hline $2.600 \mathrm{E}+02$ & $9.709 \mathrm{E}-02$ & $9.724 E-02$ & $9.483 \mathrm{E}-02$ \\
\hline $2.700 \mathrm{E}+02$ & $8.578 \mathrm{E}-02$ & $8.593 E-02$ & $8.433 E-02$ \\
\hline $2.800 E+02$ & $8.211 \mathrm{E}-02$ & $8.226 \mathrm{E}-02$ & $8.212 E-02$ \\
\hline $2.900 E+02$ & $8.239 \mathrm{E}-02$ & $8.254 \mathrm{E}-02$ & $8.371 E-02$ \\
\hline $3.000 \mathrm{E}+02$ & $8.431 \mathrm{E}-02$ & $8.446 \mathrm{E}-02$ & $8.622 \mathrm{E}-02$ \\
\hline $3.100 E+02$ & $8.665 E-02$ & $8.680 \mathrm{E}-02$ & $8.829 E-02$ \\
\hline $3.200 \mathrm{E}+02$ & $8.888 \mathrm{E}-02$ & $8.903 E-02$ & $8.966 \mathrm{E}-02$ \\
\hline $3.300 \mathrm{E}+02$ & $9.082 \mathrm{E}-02$ & $9.097 \mathrm{E}-02$ & $9.060 \mathrm{E}-02$ \\
\hline $3.400 \mathrm{E}+02$ & $9.244 \mathrm{E}-02$ & $9.259 \mathrm{E}-02$ & $9.147 \mathrm{E}-02$ \\
\hline $3.500 \mathrm{E}+02$ & $9.378 \mathrm{E}-02$ & $9.393 \mathrm{E}-02$ & $9.251 \mathrm{E}-02$ \\
\hline
\end{tabular}


Table 2-5. Continued.

\begin{tabular}{cccc}
\hline \hline $\begin{array}{c}\text { Distance } \\
\mathbf{x}(\mathrm{m})\end{array}$ & FRACVAL & EXACT $^{*}$ & STEFH** $^{*}$ \\
\hline $3.600 \mathrm{E}+02$ & 9.4 j8E -02 & $9.503 \mathrm{E}-02$ & $9.375 \mathrm{E}-02$ \\
$3.700 \mathrm{E}+02$ & $9.578 \mathrm{E}-02$ & $9.593 \mathrm{E}-02$ & $9.510 \mathrm{E}-02$ \\
$3.800 \mathrm{E}+02$ & $9.652 \mathrm{E}-02$ & $9.667 \mathrm{E}-02$ & $9.638 \mathrm{E}-02$ \\
$3.900 \mathrm{E}+02$ & $9.712 \mathrm{E}-02$ & $9.727 \mathrm{E}-02$ & $9.746 \mathrm{E}-02$ \\
$4.000 \mathrm{E}+02$ & $9.762 \mathrm{E}-02$ & $9.777 \mathrm{E}-02$ & $9.824 \mathrm{E}-02$ \\
\hline
\end{tabular}

- EXACT is the name of the mathematical model

(van Genuchten and Alves, 1982)

**. Based on Stefhest, 1970 
Table 2-6. Case 1B Results: Concentration of Cs-135 at Time $t=3,000$ Years, Zero Matrix Diffusion, Band

Release Mode, Retardation Factor $R=100$.

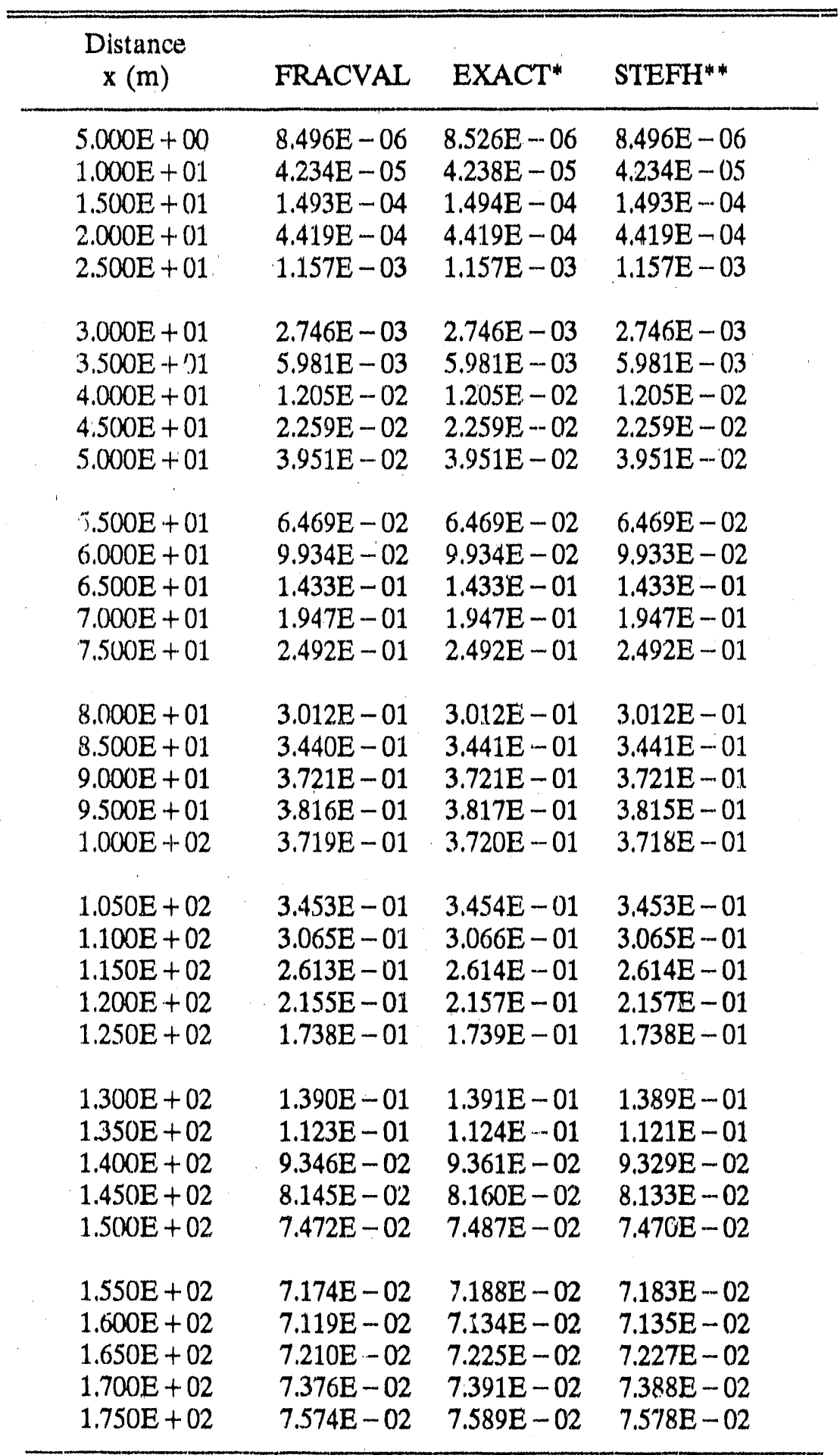


Table 2-6. Continued.

\begin{tabular}{|c|c|c|c|}
\hline $\begin{array}{c}\text { Distance } \\
\mathrm{x}(\mathrm{m})\end{array}$ & FRACVAL & EXAC'"* & STEFH** \\
\hline $1: 800 \mathrm{E}+02$ & $7.780 \mathrm{E}-02$ & $7.795 \mathrm{E}-02$ & $7.774 E-02$ \\
\hline $1.850 \mathrm{E}+02$ & $7.979 \mathrm{E}-02$ & $7.994 \mathrm{E}-02$ & $7.967 \mathrm{E}-02$ \\
\hline $1.900 \mathrm{E}+02$ & $8.165 \mathrm{E}-02$ & $8.180 \mathrm{E}-02$ & $8.150 \mathrm{E}-02$ \\
\hline $1.950 \mathrm{E}+02$ & $8.336 \mathrm{E}-02$ & $8.351 E-02$ & $8.323 E-02$ \\
\hline $2.000 \mathrm{E}+02$ & $8.492 E-02$ & $8.507 \mathrm{E}-02$ & $8.484 \mathrm{E}-02$ \\
\hline $2.050 \mathrm{E}+02$ & $8.634 \mathrm{E}-02$ & $8.649 E-02$ & $8.632 E-02$ \\
\hline $2.100 \mathrm{E}+02$ & $8.762 \mathrm{E}-02$ & $8.777 \mathrm{E} \cdots 02$ & $8.766 \mathrm{E}-02$ \\
\hline $2.150 \mathrm{E}+02$ & $8.879 \mathrm{E}-02$ & $8.894 \mathrm{E}-02$ & $8.886 \mathrm{E}-02$ \\
\hline $2.200 \mathrm{E}+02$ & $8.984 \mathrm{E}-02$ & $8.9 .99 E-02$ & $8.993 \mathrm{E}-02$ \\
\hline $2.250 \mathrm{E}+02$ & $9.079 \mathrm{E}-02$ & $9.094 \mathrm{E}-02$ & $9.088 \mathrm{E}-02$ \\
\hline $2.300 \mathrm{E}+02$ & $9.165 \mathrm{E}-02$ & $9.180 \mathrm{E}-02$ & $9.172 E-02$ \\
\hline $2.350 \mathrm{E}+02$ & $9.243 \mathrm{E}-02$ & $9.258 \mathrm{E}-02$ & $9.247 E-12$ \\
\hline $2.400 \mathrm{E}+02$ & $9.314 \mathrm{E}-02$ & $9.329 \mathrm{E}-02$ & $9.314 E-02$ \\
\hline $2.450 \mathrm{E}+02$ & $9.378 \mathrm{E}-02$ & $9.393 \mathrm{E}-02$ & $9.375 E-02$ \\
\hline $2.500 \mathrm{E}+02$ & $9.436 \mathrm{E}-02$ & $9.451 \mathrm{E}-02$ & $9.431 \mathrm{E}-02$ \\
\hline $2.550 \mathrm{E}+02$ & $9.488 \mathrm{E}-02$ & $9.503 E-02$ & $9.483 \mathrm{E}-02$ \\
\hline $2.600 \mathrm{E}+02$ & $9.535 E-02$ & $9.550 \mathrm{E}-02$ & $9.530 \mathrm{E}-02$ \\
\hline $2.650 \mathrm{E}+02$ & $9.578 \mathrm{E}-02$ & $9.593 E-02$ & $9.574 E-02$ \\
\hline $2.700 \mathrm{E}+02$ & $9.617 \mathrm{E}-02$ & $9.632 \mathrm{E}-02$ & $9.614 E-02$ \\
\hline $2.750 \mathrm{E}+02$ & $9.652 \mathrm{E}-02$ & $9.667 \mathrm{E}-02$ & $9.651 E-02$ \\
\hline $2.800 \mathrm{E}+02$ & $9.683 \mathrm{E}-02$ & $9.698 \mathrm{E}-02$ & $9.684 \mathrm{E}-02$ \\
\hline $2.850 \mathrm{E}+02$ & $9.712 \mathrm{E}-02$ & $9.727 \mathrm{E}-02$ & $9.713 E-02$ \\
\hline $2.900 \mathrm{E}+02$ & $9.738 \mathrm{E}-02$ & $9.753 E-02$ & $9.740 \mathrm{E}-02$ \\
\hline $2.950 \mathrm{E}+02$ & $9.762 \mathrm{E}-02$ & $9.777 \mathrm{E}-02$ & $9.764 E-02$ \\
\hline $3.000 \mathrm{E}+02$ & $9.783 E-02$ & $9.798 \mathrm{E}-02$ & $9.785 E-02$ \\
\hline $3.050 \mathrm{E}+02$ & $9.802 \mathrm{E}-02$ & $9.817 \mathrm{E}-02$ & $9.804 \mathrm{E}-02$ \\
\hline $3.100 \mathrm{E}+02$ & $9.819 \mathrm{E}-02$ & $9.835 E-02$ & $9.821 \mathrm{E}-02$ \\
\hline $3.150 \mathrm{E}+02$ & $9.835 \mathrm{E}-02$ & $9.850 \mathrm{E}-02$ & $9.836 \mathrm{E}-02$ \\
\hline $3.200 \mathrm{E}+02$ & $9.849 \mathrm{E}-02$ & $9.865 \mathrm{E}-02$ & $9.850 E-02$ \\
\hline $3.250 \mathrm{E}+02$ & $9.862 \mathrm{E}-02$ & $9.877 \mathrm{E}-02$ & $9.862 E-02$ \\
\hline $3,300 \mathrm{E}+02$ & $9.874 \mathrm{E}-02$ & $9.889 \mathrm{E}-02$ & $9.874 E-02$ \\
\hline $3.350 \mathrm{E}+02$ & $9.885 E-02$ & $9.900 \mathrm{E},-02$ & $9.884 \mathrm{E}-02$ \\
\hline $3.400 \mathrm{E}+02$ & $9.894 \mathrm{E}-02$ & $9.909 E-02$ & $9.893 E-02$ \\
\hline $3.450 \mathrm{E}+02$ & $9.903 \mathrm{E}-02$ & $9.918 \mathrm{E}-02$ & $9.902 E-02$ \\
\hline $3.500 \mathrm{E}+02$ & $9.911 \mathrm{E}-02$ & $9.926 \mathrm{E}-02$ & $9.910 E-02$ \\
\hline
\end{tabular}


Table 2.6. Continued.

\begin{tabular}{cccl}
\hline $\begin{array}{c}\text { Distance } \\
x(m)\end{array}$ & FRACVAL & EXACT* & STEFH** \\
\hline $3.550 \mathrm{E}+02$ & $9.918 \mathrm{E}-02$ & $9.933 \mathrm{E}-02$ & $9.917 \mathrm{E}-02$ \\
$3.600 \mathrm{E}+02$ & $9.924 \mathrm{E}-02$ & $9.939 \mathrm{E}-02$ & $9.924 \mathrm{E}-02$ \\
$3.650 \mathrm{E}+02$ & $9.930 \mathrm{E}-02$ & $9.945 \mathrm{E}-02$ & $9.930 \mathrm{E}-02$ \\
$3.700 \mathrm{E}+02$ & $9.935 \mathrm{E}-02$ & $9.950 \mathrm{E}-02$ & $9.935 \mathrm{E}-02$ \\
$3.750 \mathrm{E}+02$ & $9.940 \mathrm{E}-02$ & $9.955 \mathrm{E}-02$ & $9.940 \mathrm{E}-02$ \\
& & & \\
$3.800 \mathrm{E}+02$ & $9.944 \mathrm{E}-02$ & $9.959 \mathrm{E}-02$ & $9.944 \mathrm{E}-02$ \\
$3.850 \mathrm{E}+02$ & $9.948 \mathrm{E}-02$ & $9.963 \mathrm{E}-02$ & $9.948 \mathrm{E}-02$ \\
$3.900 \mathrm{E}+02$ & $9.952 \mathrm{E}-02$ & $9.967 \mathrm{E}-02$ & $9.952 \mathrm{E}-02$ \\
$3.950 \mathrm{E}+02$ & $9.955 \mathrm{E}-02$ & $9.970 \mathrm{E}-02$ & $9.955 \mathrm{E}-02$ \\
$4.000 \mathrm{E}+02$ & $9.958 \mathrm{E}-02$ & $9.973 \mathrm{E}-02$ & $9.958 \mathrm{E}-02$ \\
\hline \hline
\end{tabular}

* EXACT is the name of the mathematical model (van Genuchten and Alves, 1982)

** Based on Stefhest, 1970 
Table 2-7. Case 1B Results: Concentration of Cs-135 at Time $t=3,000$ Years, Zero Matrix Diffusion, Band Release Mode, Retardation Factor $R=200$.

\begin{tabular}{|c|c|c|c|}
\hline $\begin{array}{c}\text { Distance } \\
\mathrm{x}(\mathrm{m})\end{array}$ & FRACVAL & EXACT' & STEFH"* \\
\hline $\begin{array}{l}4.000 \mathrm{E}+00 \\
8.000 \mathrm{E}+00 \\
1.200 \mathrm{E}+01 \\
1.600 \mathrm{E}+01 \\
2.000 \mathrm{E}+01\end{array}$ & $\begin{array}{l}6.107 E-04 \\
2.436 E-03 \\
6.778 E-03 \\
1.558 E-02 \\
3.125 E-02\end{array}$ & $\begin{array}{l}6.106 \mathrm{E}-04 \\
2.436 \mathrm{E}-03 \\
6.776 \mathrm{E}-03 \\
1.558 \mathrm{E}-02 \\
3.125 \mathrm{E}-02\end{array}$ & $\begin{array}{l}6.107 E-04 \\
2.436 E-03 \\
6.778 E-03 \\
1.558 E-02 \\
3.125 E-02\end{array}$ \\
\hline $\begin{array}{l}2.400 \mathrm{E}+01 \\
2.800 \mathrm{E}+01 \\
3.200 \mathrm{E}+01 \\
3.600 \mathrm{E}+01 \\
4.000 \mathrm{E}+01\end{array}$ & $\begin{array}{l}5.596 \mathrm{E}-02 \\
9.070 \mathrm{E}-02 \\
1.341 \mathrm{E}-01 \\
1.820 \mathrm{E}-01 \\
2.274 \mathrm{E}-01\end{array}$ & $\begin{array}{l}5.596 \mathrm{E}-02 \\
9.071 \mathrm{E}-02 \\
1.341 \mathrm{E}-01 \\
1.820 \mathrm{E}-01 \\
2.275 \mathrm{E}-01\end{array}$ & $\begin{array}{l}5.596 \mathrm{E}-02 \\
9.070 \mathrm{E}-02 \\
1.341 \mathrm{E}-01 \\
1.820 \mathrm{E}-01 \\
2.274 \mathrm{E}-01\end{array}$ \\
\hline $\begin{array}{l}4.400 E+01 \\
4.800 E+01 \\
5.200 E+01 \\
5.600 E+01 \\
6.0(10 E+01\end{array}$ & $\begin{array}{l}2.628 \mathrm{E}-01 \\
2.818 \mathrm{E}-01 \\
2,812 \mathrm{E}-01 \\
2.6^{\prime} 23 \mathrm{E}-01 \\
2.300 \mathrm{E}-01\end{array}$ & $\begin{array}{l}2.629 E-01 \\
2.818 E-01 \\
2.812 E-01 \\
2.624 E-01 \\
2.301 E-01\end{array}$ & $\begin{array}{l}2.628 E-01 \\
2.818 E-01 \\
2.812 E-01 \\
2.623 E-01 \\
2.300 E-01\end{array}$ \\
\hline $\begin{array}{l}6.400 \mathrm{E}+01 \\
6.800 \mathrm{E}+01 \\
7.200 \mathrm{E}+01 \\
7.600 \mathrm{E}+01 \\
8.000 \mathrm{E}+01\end{array}$ & $\begin{array}{l}1.911 E-01 \\
1.525 E-01 \\
1.191 E-01 \\
9.353 E-02 \\
7.616 E-02\end{array}$ & $\begin{array}{l}1.912 E-01 \\
1.526 \mathrm{E}-01 \\
1.192 \mathrm{E}-01 \\
9.367 \mathrm{E}-02 \\
7.631 \mathrm{E}-02\end{array}$ & $\begin{array}{l}1.911 E-01 \\
1.525 E-01 \\
1.191 E-01 \\
9.353 E-02 \\
7.616 E-02\end{array}$ \\
\hline $\begin{array}{l}8.400 \mathrm{E}+01 \\
8.800 \mathrm{E}+01 \\
9.200 \mathrm{E}+01 \\
9.600 \mathrm{E}+01 \\
1.000 \mathrm{E}+02\end{array}$ & $\begin{array}{l}6.590 E-02 \\
6.098 E-02 \\
5.964 E-02 \\
6.041 E-02 \\
6.229 E-02\end{array}$ & $\begin{array}{l}6.605 E-02 \\
6.113 E-02 \\
5.979 E-02 \\
6.056 E-02 \\
6.244 E-02\end{array}$ & $\begin{array}{l}6.590 E-02 \\
6.099 E-02 \\
5.964 E-02 \\
6.041 E-02 \\
6.229 E-02\end{array}$ \\
\hline $\begin{array}{l}1.040 \mathrm{E}+02 \\
1.080 \mathrm{E}+02 \\
1.120 \mathrm{E}+02 \\
1.160 \mathrm{E}+02 \\
1.200 \mathrm{E}+02\end{array}$ & $\begin{array}{l}6.464 E-02 \\
6.711 E-02 \\
6.953 E-02 \\
7.183 E-02 \\
7.397 E-02\end{array}$ & $\begin{array}{l}6.479 E-02 \\
6.726 E-02 \\
6.969 E-02 \\
7.198 E-02 \\
7.412 E-02\end{array}$ & $\begin{array}{l}6.463 E-02 \\
6.711 E-02 \\
6.953 E-02 \\
7.183 E-02 \\
7.397 E-02\end{array}$ \\
\hline $\begin{array}{l}1.240 \mathrm{E}+02 \\
1.280 \mathrm{E}+02 \\
1.320 \mathrm{E}+02 \\
1.360 \mathrm{E}+02 \\
1.400 \mathrm{E}+02\end{array}$ & $\begin{array}{l}7.596 \mathrm{E}-02 \\
7.779 \mathrm{E}-02 \\
7.949 \mathrm{E}-02 \\
8.105 \mathrm{E}-02 \\
8.250 \mathrm{E}-02\end{array}$ & $\begin{array}{l}7.611 \mathrm{E}-02 \\
7.794 \mathrm{E}-02 \\
7.964 \mathrm{E}-02 \\
8.120 \mathrm{E}-02 \\
8.265 \mathrm{E}-02\end{array}$ & $\begin{array}{l}7.596 \mathrm{E}-02 \\
7.780 \mathrm{E}-02 \\
7.949 \mathrm{E}-02 \\
8.105 \mathrm{E}-02 \\
8.250 \mathrm{E}-02\end{array}$ \\
\hline
\end{tabular}


Tuble 2-7. Continued.

\begin{tabular}{|c|c|c|c|}
\hline $\begin{array}{c}\text { Distance } \\
\times(n 1)\end{array}$ & FRACVAL & EXACT* & STEFH** \\
\hline $\begin{array}{l}1.440 \mathrm{E}+02 \\
1.480 \mathrm{E}+02 \\
1.520 \mathrm{E}+02 \\
1.560 \mathrm{E}+02 \\
1.600 \mathrm{E}+02\end{array}$ & $\begin{array}{l}8.383 E-02 \\
8.506 E-02 \\
8.620 E-02 \\
8.725 E-02 \\
8.822 E-02\end{array}$ & $\begin{array}{l}8.398 E-02 \\
8.521 E-02 \\
8.635 E-02 \\
8.740 E-02 \\
8.837 E-02\end{array}$ & $\begin{array}{l}8.383 E-02 \\
8.506 E-02 \\
8.620 E-02 \\
8.725 E-02 \\
8.822 E-02\end{array}$ \\
\hline $\begin{array}{l}1.640 \mathrm{E}+02 \\
1.680 \mathrm{E}+02 \\
1.720 \mathrm{E}+02 \\
1.760 \mathrm{E}+02 \\
1.800 \mathrm{E}+02\end{array}$ & $\begin{array}{l}8.911 E-02 \\
8.994 E-02 \\
9.070 E-02 \\
9.140 E-02 \\
9.205 E-02\end{array}$ & $\begin{array}{l}3.926 \mathrm{E}-02 \\
2.009 \mathrm{E}-02 \\
9.085 \mathrm{E}-02 \\
9.155 \mathrm{E}-02 \\
9.220 \mathrm{E}-02\end{array}$ & $\begin{array}{l}8.911 \mathrm{E}-02 \\
8.994 \mathrm{E}-02 \\
9.070 \mathrm{E}-02 \\
9.140 \mathrm{E}-02 \\
9.205 \mathrm{E}-02\end{array}$ \\
\hline $\begin{array}{l}1.840 \mathrm{E}+02 \\
1.880 \mathrm{E}+02 \\
1.920 \mathrm{E}+02 \\
1.960 \mathrm{E}+02 \\
2.000 \mathrm{E}+02\end{array}$ & $\begin{array}{l}9.265 E-02 \\
9.321 E-02 \\
9.372 E-02 \\
9.419 E-02 \\
9.462 E-02\end{array}$ & $\begin{array}{l}9.280 \mathrm{E}-02 \\
9.336 \mathrm{E}-02 \\
9.387 \mathrm{E}-02 \\
9.434 \mathrm{E}-02 \\
9.477 \mathrm{E}-02\end{array}$ & $\begin{array}{l}9.265 E-02 \\
9.321 E-02 \\
9.372 E-02 \\
9.419 E-02 \\
9.462 E-02\end{array}$ \\
\hline $\begin{array}{l}2.040 \mathrm{E}+02 \\
2.080 \mathrm{E}+02 \\
2.120 \mathrm{E}+02 \\
2.160 \mathrm{E}+02 \\
2.200 \mathrm{E}+02\end{array}$ & $\begin{array}{l}9.503 E-02 \\
9.540 E-02 \\
9.574 E-02 \\
9.605 E-02 \\
9.635 E-02\end{array}$ & $\begin{array}{l}9.518 \mathrm{E}-02 \\
9.555 \mathrm{E}-02 \\
9.589 \mathrm{E}-02 \\
9.621 \mathrm{E}-02 \\
9.650 \mathrm{E}-02\end{array}$ & $\begin{array}{l}9.502 E-02 \\
9.540 E-02 \\
9.574 E-02 \\
9.605 E-02 \\
9.635 E-02\end{array}$ \\
\hline $\begin{array}{l}2.240 \mathrm{E}+02 \\
2.280 \mathrm{E}+02 \\
2.320 \mathrm{E}+02 \\
2.360 \mathrm{E}+02 \\
2.400 \mathrm{E}+02\end{array}$ & $\begin{array}{l}9.662 E-02 \\
9.686 E-02 \\
9.709 E-02 \\
9.731 E-02 \\
9.750 E-02\end{array}$ & $\begin{array}{l}9.677 E-02 \\
9.701 E-02 \\
9.724 E-02 \\
9.746 E-02 \\
9.765 E-02\end{array}$ & $\begin{array}{l}9.662 E-02 \\
9.686 E-02 \\
9.709 E-02 \\
9.731 E-02 \\
9.750 E-02\end{array}$ \\
\hline $\begin{array}{l}2.440 \mathrm{E}+02 \\
2.480 \mathrm{E}+02 \\
2.520 \mathrm{E}+02 \\
2.550 \mathrm{E}+02 \\
2.600 \mathrm{E}+02\end{array}$ & $\begin{array}{l}9.768 E-02 \\
9.785 E-02 \\
9.800 E-02 \\
9.814 E-02 \\
9.828 E-02\end{array}$ & $\begin{array}{l}9.783 \mathrm{E}-02 \\
9.800 \mathrm{E}-02 \\
9.815 \mathrm{E}-02 \\
9.829 \mathrm{E}-02 \\
9.843 \mathrm{E}-02\end{array}$ & $\begin{array}{l}9.768 E-02 \\
9.785 E-02 \\
9.800 E-02 \\
9.814 E-02 \\
9.828 E-02\end{array}$ \\
\hline $\begin{array}{l}2.640 \mathrm{E}+02 \\
2.680 \mathrm{E}+02 \\
2.720 \mathrm{E}+02 \\
2.760 \mathrm{E}+02 \\
2.800 \mathrm{E}+02\end{array}$ & $\begin{array}{l}9.840 E-02 \\
9.851 E-02 \\
9.861 E-02 \\
9.871 E-02 \\
9.879 E-02\end{array}$ & $\begin{array}{l}9.855 \mathrm{E}-02 \\
9.866 \mathrm{E}-02 \\
9.876 \mathrm{E}-02 \\
9.886 \mathrm{E}-02 \\
9.894 \mathrm{E}-02\end{array}$ & $\begin{array}{l}9.840 E-02 \\
9.851 E-02 \\
9.861 E-02 \\
9.871 E-02 \\
9.879 E-02\end{array}$ \\
\hline
\end{tabular}


Table 2.7. Continued.

\begin{tabular}{cccc}
\hline $\begin{array}{c}\text { Distance } \\
\text { X }(\mathrm{m})\end{array}$ & FRACVAL & EXACT* & STEFH \\
\hline $2.840 \mathrm{E}+02$ & $9.888 \mathrm{E}-02$ & $9.903 \mathrm{E}-02$ & $9.888 \mathrm{E}-02$ \\
$2.880 \mathrm{E}+02$ & $9.895 \mathrm{E}-02$ & $9.910 \mathrm{E}-02$ & $9.895 \mathrm{E}-02$ \\
$2.920 \mathrm{E}+02$ & $9.902 \mathrm{E}-02$ & $9.917 \mathrm{E}-02$ & $9.902 \mathrm{E}-02$ \\
$2.960 \mathrm{E}+02$ & $9.908 \mathrm{E}-02$ & $9.923 \mathrm{E}-02$ & $9.908 \mathrm{E}-02$ \\
$3.000 \mathrm{E}+02$ & $9.914 \mathrm{E}-02$ & $9.929 \mathrm{E}-02$ & $9.914 \mathrm{E}-02$ \\
& & & \\
$3.040 \mathrm{E}+02$ & $9.920 \mathrm{E}-02$ & $9.935 \mathrm{E}-02$ & $9.920 \mathrm{E}-02$ \\
$3.080 \mathrm{E}+02$ & $9.925 \mathrm{E}-02$ & $9.940 \mathrm{E}-02$ & $9.925 \mathrm{E}-02$ \\
$3.120 \mathrm{E}+02$ & $9.929 \mathrm{E}-02$ & $9.944 \mathrm{E}-02$ & $9.929 \mathrm{E}-02$ \\
$3.160 \mathrm{E}+02$ & $9.934 \mathrm{E}-02$ & $9.949 \mathrm{E}-02$ & $9.934 \mathrm{E}-02$ \\
$3.200 \mathrm{E}+02$ & $9.938 \mathrm{E}-02$ & $9.953 \mathrm{E}-02$ & $9.938 \mathrm{E}-02$ \\
& & & \\
$3.240 \mathrm{E}+02$ & $9.941 \mathrm{E}-02$ & $9.956 \mathrm{E}-02$ & $9.941 \mathrm{E}-02$ \\
$3.280 \mathrm{E}+02$ & $9.945 \mathrm{E}-02$ & $9.960 \mathrm{E}-02$ & $9.945 \mathrm{E}-02$ \\
$3.320 \mathrm{E}+02$ & $9.948 \mathrm{E}-02$ & $9.963 \mathrm{E}-02$ & $9.948 \mathrm{E}-02$ \\
$3.360 \mathrm{E}+02$ & $9.951 \mathrm{E}-02$ & $9.966 \mathrm{E}-02$ & $9.951 \mathrm{E}-02$ \\
$3.400 \mathrm{E}+02$ & $9.953 \mathrm{E}-02$ & $9.968 \mathrm{E}-02$ & $9.953 \mathrm{E}-02$ \\
& & & \\
$3.440 \mathrm{E}+02$ & $9.956 \mathrm{E}-02$ & $9.971 \mathrm{E}-02$ & $9.956 \mathrm{E}-02$ \\
$3.480 \mathrm{E}+02$ & $9.958 \mathrm{E}-02$ & $9.973 \mathrm{E}-02$ & $9.958 \mathrm{E}-02$ \\
$3.520 \mathrm{E}+02$ & $9.960 \mathrm{E}-02$ & $9.975 \mathrm{E}-02$ & $9.960 \mathrm{E}-02$ \\
$3.560 \mathrm{E}+02$ & $9.962 \mathrm{E}-02$ & $9.977 \mathrm{E}-02$ & $9.962 \mathrm{E}-02$ \\
$3.600 \mathrm{E}+02$ & $9.964 \mathrm{E}-02$ & $9.979 \mathrm{E}-02$ & $9.964 \mathrm{E}-02$ \\
& & & \\
$3.640 \mathrm{E}+02$ & $9.965 \mathrm{E}-02$ & $9.980 \mathrm{E}-02$ & $9.965 \mathrm{E}-02$ \\
$3.680 \mathrm{E}+02$ & $9.967 \mathrm{E}-02$ & $9.982 \mathrm{E}-02$ & $9.967 \mathrm{E}-02$ \\
$3.720 \mathrm{E}+02$ & $9.968 \mathrm{E}-02$ & $9.983 \mathrm{E}-02$ & $9.968 \mathrm{E}-02$ \\
$3.760 \mathrm{E}+02$ & $9.969 \mathrm{E}-02$ & $9.985 \mathrm{E}-02$ & $9.969 \mathrm{E}-02$ \\
$3.800 \mathrm{E}+02$ & $9.971 \mathrm{E}-02$ & $9.986 \mathrm{E}-02$ & $9.971 \mathrm{E}-02$ \\
& & & \\
$3.840 \mathrm{E}+02$ & $9.972 \mathrm{E}-02$ & $9.987 \mathrm{E}-02$ & $9.972 \mathrm{E}-02$ \\
$3.880 \mathrm{E}+02$ & $9.973 \mathrm{E}-02$ & $9.988 \mathrm{E}-02$ & $9.973 \mathrm{E}-02$ \\
$3.920 \mathrm{E}+02$ & $9.974 \mathrm{E}-02$ & $9.989 \mathrm{E}-02$ & $9.974 \mathrm{E}-02$ \\
$3.960 \mathrm{E}+02$ & $9.975 \mathrm{E}-02$ & $9.990 \mathrm{E}-02$ & $9.975 \mathrm{E}-02$ \\
$4.000 \mathrm{E}+02$ & $9.975 \mathrm{E}-02$ & $9.990 \mathrm{E}-02$ & $9.975 \mathrm{E}-02$ \\
\hline \hline
\end{tabular}

- EXACT is the name of the mathematical model (van Genuchten and Alves, 1982)

"* Based on Stefhest, 1970 


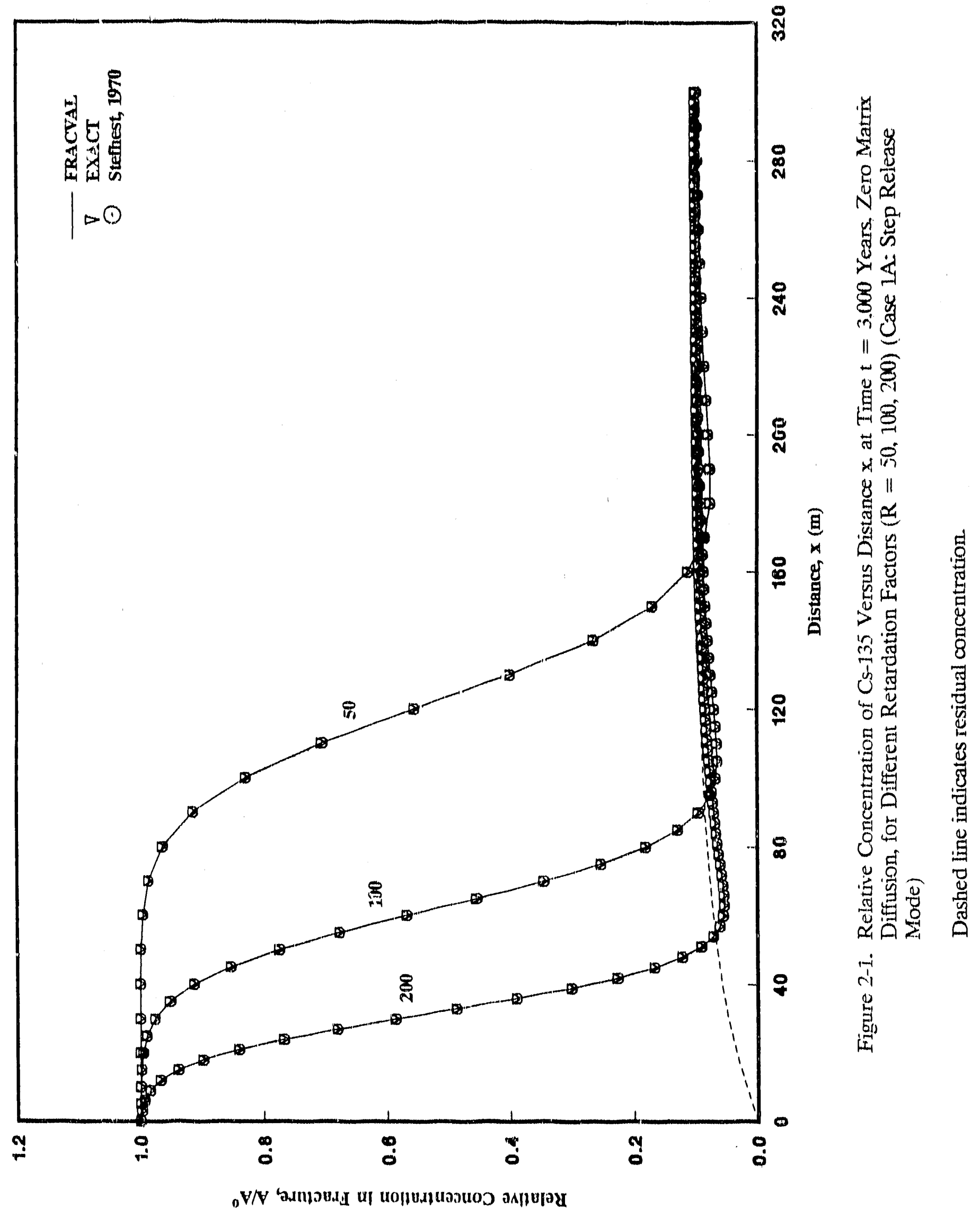




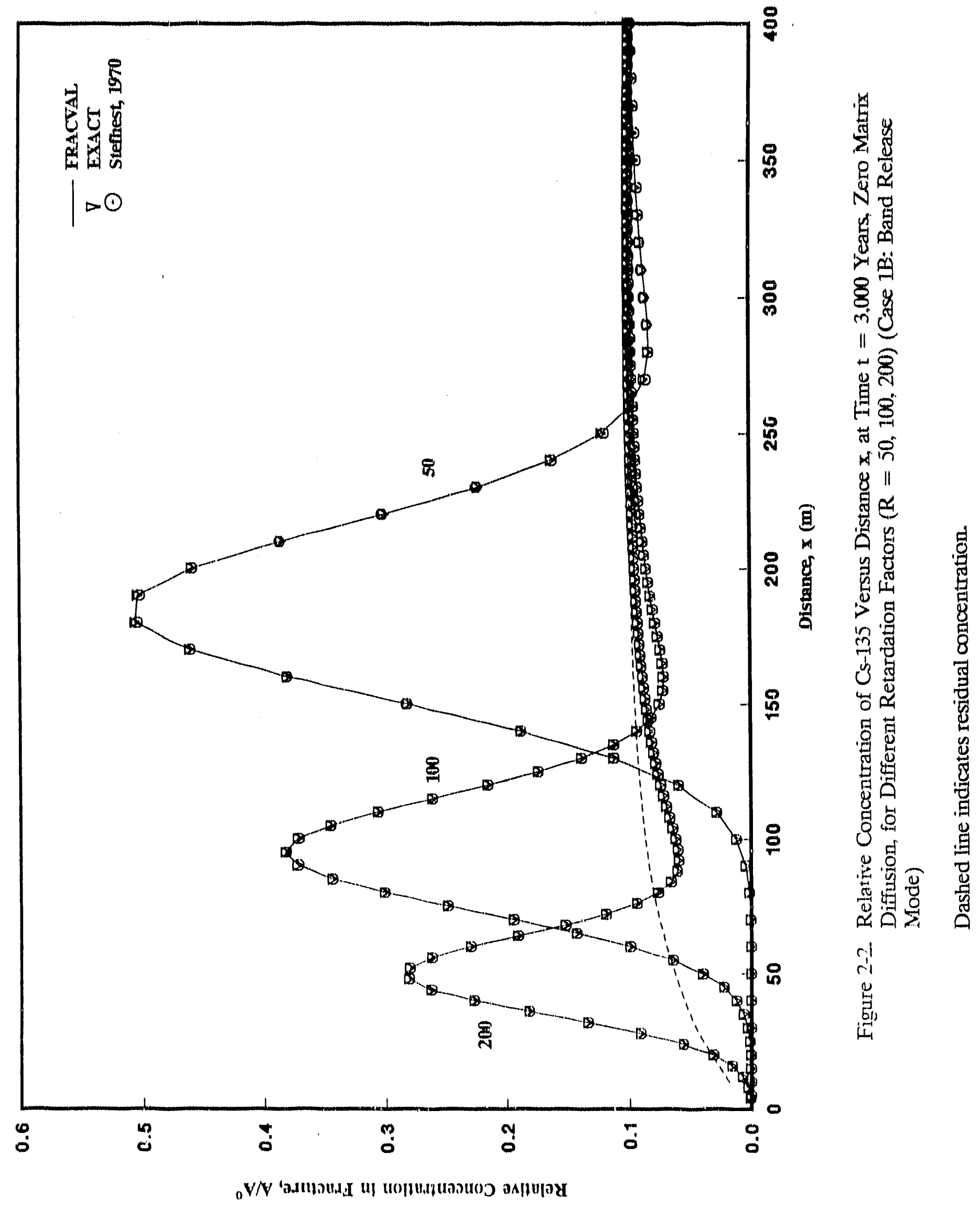


'Tablo 2.8. Input Parmotors for Caso 2

\begin{tabular}{|c|c|}
\hline Spocias & Np.237 \\
\hline 'T'ypo ol' Rulansos Modo & Stop \\
\hline 'T'1/2 & $2.14 \times 10^{11} \mathrm{yr}$ \\
\hline$\Lambda^{(1) *}$ & 1,0 \\
\hline$u_{1}^{*}$ & 0.0 \\
\hline$\| z^{*}$ & 0.0 \\
\hline a & 0.0 \\
\hline$b_{1}^{*}$ & 0.0 \\
\hline$D_{p}$ & $0.01 \mathrm{~m}^{2} / \mathrm{yr}$ \\
\hline$u$ & $10.0 \mathrm{~m} / \mathrm{yr}$ \\
\hline$D_{x}$ & $1.0 \mathrm{~m}^{2} / \mathrm{yr}$ \\
\hline b) & $0.005 \mathrm{~m}$ \\
\hline R & 1.0 \\
\hline$R^{\prime}$ & $50,100,200$ \\
\hline
\end{tabular}

*(urbilrury units of activily/l, i) 
Tuble 249, Case 2 Results: Concentration of Np-237 in the Fracture, at Time $t=10,000$ Years, Stop ki lesase Mode, Retardation Factor R 1

\begin{tabular}{cccc}
\hline $\begin{array}{c}\text { Distance } \\
\mathbf{x}(\mathrm{m})\end{array}$ & FRACVAI, & \multicolumn{3}{c}{ STEFH } & FRACFLO \\
\hline $1.000 \mathrm{E}+01$ & $9.956 \mathrm{E}-01$ & $9.956 \mathrm{E}-01$ & $9.956 \mathrm{E}-01$ \\
$1.500 \mathrm{E}+01$ & $9.951 \mathrm{E}-01$ & $9.951 \mathrm{E}-01$ & $9.951 \mathrm{E}-01$ \\
$2.000 \mathrm{E}+01$ & $9.945 \mathrm{E}-01$ & $9.945 \mathrm{E}-01$ & $9.945 \mathrm{E}-01$ \\
$3.000 \mathrm{E}+01$ & $9.934 \mathrm{E}-01$ & $9.934 \mathrm{E}-01$ & $9.934 \mathrm{E}-01$ \\
$4.000 \mathrm{E}+01$ & $9.923 \mathrm{E}-01$ & $9.923 \mathrm{E}-01$ & $9.923 \mathrm{E}-01$ \\
& & & \\
$5.000 \mathrm{E}+01$ & $9.911 \mathrm{E}-01$ & $9.911 \mathrm{E}-01$ & $9.911 \mathrm{E}-01$ \\
$6.000 \mathrm{E}+01$ & $9.900 \mathrm{E}-01$ & $9.900 \mathrm{E}-01$ & $9.900 \mathrm{E}-01$ \\
$7.000 \mathrm{E}+01$ & $9.889 \mathrm{E}-01$ & $9.889 \mathrm{E}-01$ & $9.889 \mathrm{E}-01$ \\
$8.000 \mathrm{E}+01$ & $9.878 \mathrm{E}-01$ & $9.878 \mathrm{E}-01$ & $9.878 \mathrm{E}-01$ \\
$9.000 \mathrm{E}+01$ & $9.866 \mathrm{E}-01$ & $9.866 \mathrm{E}-01$ & $9.866 \mathrm{E}-01$ \\
& & & \\
$1.000 \mathrm{E}+02$ & $9.855 \mathrm{E}-01$ & $9.855 \mathrm{E}-01$ & $9.855 \mathrm{E}-01$ \\
$1.500 \mathrm{E}+02$ & $9.799 \mathrm{E}-01$ & $9.799 \mathrm{E}-01$ & $9.799 \mathrm{E}-01$ \\
$2.000 \mathrm{E}+02$ & $9.743 \mathrm{E}-01$ & $9.743 \mathrm{E}-01$ & $9.743 \mathrm{E}-01$ \\
$3.000 \mathrm{E}+02$ & $9.630 \mathrm{E}-01$ & $9.630 \mathrm{E}-01$ & $9.630 \mathrm{E}-01$ \\
$4.000 \mathrm{E}+02$ & $9.517 \mathrm{E}-01$ & $9.517 \mathrm{E}-01$ & $9.517 \mathrm{E}-01$ \\
& & & \\
$5.000 \mathrm{E}+02$ & $9.404 \mathrm{E}-01$ & $9.404 \mathrm{E}-01$ & $9.404 \mathrm{E}-01$ \\
$6.000 \mathrm{E}+02$ & $9.292 \mathrm{E}-01$ & $9.292 \mathrm{E}-01$ & $9.292 \mathrm{E}-01$ \\
$7.000 \mathrm{E}+02$ & $9.179 \mathrm{E}-01$ & $9.179 \mathrm{E}-01$ & $9.179 \mathrm{E}-01$ \\
$8.000 \mathrm{E}+02$ & $9.066 \mathrm{E}-01$ & $9.066 \mathrm{E}-01$ & $9.066 \mathrm{E}-01$ \\
$9.000 \mathrm{E}+02$ & $8.954 \mathrm{E}-01$ & $8.954 \mathrm{E}-01$ & $8.954 \mathrm{E}-01$ \\
& & & \\
$1.000 \mathrm{E}+03$ & $8.841 \mathrm{E}-01$ & $8.841 \mathrm{E}-01$ & $8.841 \mathrm{E}-01$ \\
$1.500 \mathrm{E}+03$ & $8.281 \mathrm{E}-01$ & $8.281 \mathrm{E}-01$ & $8.281 \mathrm{E}-01$ \\
$2.000 \mathrm{E}+03$ & $7.726 \mathrm{E}-01$ & $7.726 \mathrm{E}-01$ & $7.726 \mathrm{E}-01$ \\
$3.000 \mathrm{E}+03$ & $6.645 \mathrm{E}-01$ & $6.645 \mathrm{E}-01$ & $6.645 \mathrm{E}-01$ \\
$4.000 \mathrm{E}+03$ & $5.619 \mathrm{E}-01$ & $5.619 \mathrm{E}-01$ & $5.619 \mathrm{E}-01$ \\
& & & \\
$5.000 \mathrm{E}+03$ & $4.667 \mathrm{E}-01$ & $4.667 \mathrm{E}-01$ & $4.667 \mathrm{E}-01$ \\
$6.000 \mathrm{E}+03$ & $3.802 \mathrm{E}-01$ & $3.802 \mathrm{E}-01$ & $3.802 \mathrm{E}-01$ \\
$7.000 \mathrm{E}+03$ & $3.037 \mathrm{E}-01$ & $3.037 \mathrm{E}-01$ & $3.037 \mathrm{E}-01$ \\
$8.000 \mathrm{E}+03$ & $2.374 \mathrm{E}-01$ & $2.374 \mathrm{E}-01$ & $2.374 \mathrm{E}-01$ \\
$9.000 \mathrm{E}+03$ & $1.815 \mathrm{E}-01$ & $1.815 \mathrm{E}-01$ & $1.815 \mathrm{E}-01$ \\
& & & \\
$1.000 \mathrm{E}+04$ & $1.356 \mathrm{E}-01$ & $1.356 \mathrm{E}-01$ & $1.356 \mathrm{E}-01$ \\
$1.500 \mathrm{E}+04$ & $2.133 \mathrm{E}-02$ & $2.133 \mathrm{E}-02$ & $2.133 \mathrm{E}-02$ \\
$2.000 \mathrm{E}+04$ & $1.561 \mathrm{E}-03$ & $1.561 \mathrm{E}-03$ & $1.561 \mathrm{E}-03$ \\
$3.000 \mathrm{E}+04$ & $3.959 \mathrm{E}-07$ & $3.963 \mathrm{E}-07$ & $3.959 \mathrm{E}-07$ \\
$4.000 \mathrm{E}+04$ & $2.842 \mathrm{E}-13$ & $-1.130 \mathrm{E}-11$ & $2.842 \mathrm{E}-13$ \\
\hline
\end{tabular}

" Based on Stefhest, 1970 
Tuble 2.10. Case 2 Results: Concentration of $\mathrm{Np}-237$ in the Fracture, at Time $t=10,000$ Years, Step Release Mode, Retardation Factor $R=100$

\begin{tabular}{cccc}
\hline $\begin{array}{c}\text { Distance } \\
\mathbf{x}(\mathrm{m})\end{array}$ & FRACVAL & \multicolumn{1}{c}{ STEFH* } & FRACFLO \\
\hline $1.000 \mathrm{E}+01$ & $9.855 \mathrm{E}-01$ & $9.855 \mathrm{E}-01$ & $9.855 \mathrm{E}-01$ \\
$1.500 \mathrm{E}+01$ & $9.799 \mathrm{E}-01$ & $9.799 \mathrm{E}-01$ & $9.799 \mathrm{E}-01$ \\
$2.000 \mathrm{E}+01$ & $9.743 \mathrm{E}-01$ & $9.743 \mathrm{E}-01$ & $9.743 \mathrm{E}-01$ \\
$3.000 \mathrm{E}+01$ & $9.630 \mathrm{E}-01$ & $9.630 \mathrm{E}-01$ & $9.630 \mathrm{E}-01$ \\
$4.000 \mathrm{E}+01$ & $9.518 \mathrm{E}-01$ & $9.518 \mathrm{E}-01$ & $9.518 \mathrm{E}-01$ \\
& & & \\
$5.000 \mathrm{E}+01$ & $9.406 \mathrm{E}-01$ & $9.406 \mathrm{E}-01$ & $9.406 \mathrm{E}-01$ \\
$6.000 \mathrm{E}+01$ & $9.293 \mathrm{E}-01$ & $9.293 \mathrm{E}-01$ & $9.293 \mathrm{E}-01$ \\
$7.000 \mathrm{E}+01$ & $9.181 \mathrm{E}-01$ & $9.181 \mathrm{E}-01$ & $9.181 \mathrm{E}-01$ \\
$8.000 \mathrm{E}+01$ & $9.069 \mathrm{E}-01$ & $9.069 \mathrm{E}-01$ & $9.069 \mathrm{E}-01$ \\
$9.000 \mathrm{E}+01$ & $8.958 \mathrm{E}-01$ & $8.958 \mathrm{E}-01$ & $8.958 \mathrm{E}-01$ \\
& & & \\
$1.000 \mathrm{E}+02$ & $8.846 \mathrm{E}-01$ & $8.846 \mathrm{E}-01$ & $8.846 \mathrm{E}-01$ \\
$1.500 \mathrm{E}+02$ & $8.292 \mathrm{E}-01$ & $8.292 \mathrm{E}-01$ & $8.292 \mathrm{E}-01$ \\
$2.000 \mathrm{E}+02$ & $7.746 \mathrm{E}-01$ & $7.746 \mathrm{E}-01$ & $7.746 \mathrm{E}-01$ \\
$3.000 \mathrm{E}+02$ & $6.688 \mathrm{E}-01$ & $6.688 \mathrm{E}-01$ & $6.688 \mathrm{E}-01$ \\
$4.000 \mathrm{E}+02$ & $5.690 \mathrm{E}-01$ & $5.690 \mathrm{E}-01$ & $5.690 \mathrm{E}-01$ \\
& & & \\
$5.000 \mathrm{E}+02$ & $4.769 \mathrm{E}-01$ & $4.769 \mathrm{E}-01$ & $4.769 \mathrm{E}-01$ \\
$6.000 \mathrm{E}+02$ & $3.935 \mathrm{E}-01$ & $3.935 \mathrm{E}-01$ & $3.935 \mathrm{E}-01$ \\
$7.000 \mathrm{E}+02$ & $3.195 \mathrm{E}-01$ & $3.195 \mathrm{E}-01$ & $3.195 \mathrm{E}-01$ \\
$8.000 \mathrm{E}+02$ & $2.552 \mathrm{E}-01$ & $2.552 \mathrm{E}-01$ & $2.552 \mathrm{E}-01$ \\
$9.000 \mathrm{E}+02$ & $2.005 \mathrm{E}-01$ & $2.005 \mathrm{E}-01$ & $2.005 \mathrm{E} \cdots 01$ \\
& & & \\
$1.000 \mathrm{E}+03$ & $1.548 \mathrm{E}-01$ & $1.548 \mathrm{E}-01$ & $1.548 \mathrm{E}-01$ \\
$1.500 \mathrm{E}+03$ & $3.251 \mathrm{E}-02$ & $3.251 \mathrm{E}-02$ & $3.251 \mathrm{E}-02$ \\
$2.000 \mathrm{E}+03$ & $4.277 \mathrm{E}-03$ & $4.277 \mathrm{E}-03$ & $4.277 \mathrm{E}-03$ \\
$3.000 \mathrm{E}+03$ & $1.664 \mathrm{E}-05$ & $1.664 \mathrm{E}-05$ & $1.664 \mathrm{E}-05$ \\
$4.000 \mathrm{E}+03$ & $7.972 \mathrm{E}-09$ & $7.972 \mathrm{E}-09$ & $9.972 \mathrm{E}-09$ \\
& & & \\
$5.000 \mathrm{E}+03$ & $4.255 \mathrm{E}-13$ & $3.524 \mathrm{E}-13$ & $4.255 \mathrm{E}-13$ \\
$6.000 \mathrm{E}+03$ & $2.322 \mathrm{E}-18$ & $-2.249 \mathrm{E}-14$ & $2.322 \mathrm{E}-18$ \\
$7.000 \mathrm{E}+03$ & $1.195 \mathrm{E}-24$ & $6.985 \mathrm{E}-16$ & $1.195 \mathrm{E}-24$ \\
$8.000 \mathrm{0}+03$ & $5.349 \mathrm{E}-32$ & $1.831 \mathrm{E}-17$ & $5.349 \mathrm{E}-32$ \\
$9.000 \mathrm{E}+03$ & $1.922 \mathrm{E}-40$ & $-2.212 \mathrm{E}-18$ & $1.922 \mathrm{E}-40$ \\
& & & \\
$1.000 \mathrm{E}+04$ & $5.102 \mathrm{E}-50$ & $-7.266 \mathrm{E}-20$ & $5.102 \mathrm{E}-50$ \\
\hline
\end{tabular}

* Based on Stefhest, 1970 
Table 2.11. Case 2 Results: Concentration of Np-237 in the Fracture, at Time $t=10,000$ Years, Step Release Mode, Retardation Factor $R=10,000$

\begin{tabular}{cccc}
\hline \hline $\begin{array}{c}\text { Distance } \\
\mathbf{x}(\mathrm{m})\end{array}$ & FRACVAL & STEFH* & FRACFLO \\
\hline $1.000 \mathrm{E}+01$ & $8.847 \mathrm{E}-01$ & $8.847 \mathrm{E}-01$ & $8.847 \mathrm{E}-01$ \\
$1.500 \mathrm{E}+01$ & $8.294 \mathrm{E}-01$ & $8.294 \mathrm{E}-01$ & $8.294 \mathrm{E}-01$ \\
$2.000 \mathrm{E}+01$ & $7.748 \mathrm{E}-01$ & $7.748 \mathrm{E}-01$ & $7.748 \mathrm{E}-01$ \\
$3.000 \mathrm{E}+01$ & $6.693 \mathrm{E}-01$ & $6.693 \mathrm{E}-01$ & $6.693 \mathrm{E}-01$ \\
$4.000 \mathrm{E}+01$ & $5.700 \mathrm{E}-01$ & $5.700 \mathrm{E}-01$ & $5.700 \mathrm{E}-01$ \\
& & & \\
$5.000 \mathrm{E}+01$ & $4.783 \mathrm{E}-01$ & $4.783 \mathrm{E}-01$ & $4.783 \mathrm{E}-01$ \\
$6.000 \mathrm{E}+01$ & $3.953 \mathrm{E}-01$ & $3.953 \mathrm{E}-01$ & $3.953 \mathrm{E}-01$ \\
$1.000 \mathrm{E}+01$ & $3.217 \mathrm{E}-01$ & $3.217 \mathrm{E}-01$ & $3.217 \mathrm{E}-01$ \\
$8.000 \mathrm{E}+01$ & $2.576 \mathrm{E}-01$ & $2.576 \mathrm{E}-01$ & $2.576 \mathrm{E}-01$ \\
$9.000 \mathrm{E}+01$ & $2.030 \mathrm{E}-01$ & $2.030 \mathrm{E}-01$ & $2.030 \mathrm{E}-01$ \\
& & & \\
$1.000 \mathrm{E}+02$ & $1.574 \mathrm{E}-01$ & $1.574 \mathrm{E}-01$ & $1.574 \mathrm{E}-01$ \\
$1.500 \mathrm{E}+02$ & $3.418 \mathrm{E}-02$ & $3.418 \mathrm{E}-02$ & $3.41 .8 \mathrm{E}-02$ \\
$2.000 \mathrm{E}+02$ & $4.786 \mathrm{E}-03$ & $4.786 \mathrm{E}-03$ & $4.786 \mathrm{E}-03$ \\
$3.000 \mathrm{E}+02$ & $2.393 \mathrm{E}-05$ & $2.393 \mathrm{E}-05$ & $2.393 \mathrm{E}-05$ \\
$4.000 \mathrm{E}+02$ & $1.863 \mathrm{E}-08$ & $1.863 \mathrm{E}-08$ & $1.863 \mathrm{E}-08$ \\
& & & \\
$5.000 \mathrm{E}+02$ & $2.218 \mathrm{E}-12$ & $2.090 \mathrm{E}-12$ & $2.218 \mathrm{E}-12$ \\
$6.000 \mathrm{E}+02$ & $4.031 \mathrm{E}-17$ & $-1.528 \mathrm{E}-14$ & $4.031 \mathrm{E}-17$ \\
$7.000 \mathrm{E}+02$ & $1.124 \mathrm{E}-22$ & $1.062 \mathrm{E}-15$ & $1.124 \mathrm{E}-22$ \\
$8.000 \mathrm{E}+02$ & $4.854 \mathrm{E}-29$ & $-3.246 \mathrm{E}-17$ & $4.854 \mathrm{E}-29$ \\
$9.000 \mathrm{E}+02$ & $3.277 \mathrm{E}-36$ & $1.737 \mathrm{E}-18$ & $3.277 \mathrm{E}-36$ \\
& & & \\
$1.000 \mathrm{E}+03$ & $3.499 \mathrm{E}-44$ & $-3.555 \mathrm{E}-19$ & $3.499 \mathrm{E}-44$ \\
\hline
\end{tabular}

" Based on Stefhesi, 1970 
Table 2-12. Case 2 Results: Concentration of Np-237 in the Rock Matrix, at Time $t=10,000$ Years, Step Release Mode, Retardation Factor $\mathbf{R}^{\prime}=1$

\begin{tabular}{|c|c|c|c|}
\hline $\begin{array}{c}\text { Distance } \\
\mathbf{x}(\mathrm{m})\end{array}$ & FRACVAL & STEFH* & FRACFLO \\
\hline $\begin{array}{l}1.000 \mathrm{E}-02 \\
1.500 \mathrm{E}-02 \\
2.000 \mathrm{E}-02 \\
3.000 \mathrm{E}-02 \\
4.000 \mathrm{E}-02\end{array}$ & $\begin{array}{l}9.852 \mathrm{E}-01 \\
9.850 \mathrm{E}-01 \\
9.847 \mathrm{E}-01 \\
9.841 \mathrm{E}-01 \\
9.835 \mathrm{E}-01\end{array}$ & $\begin{array}{l}9.852 \mathrm{E}-01 \\
9.850 \mathrm{E}-01 \\
0.847 \mathrm{E}-01 \\
9.841 \mathrm{E}-01 \\
9.835 \mathrm{E}-01\end{array}$ & $\begin{array}{l}9.852 \mathrm{E}-01 \\
9.850 \mathrm{E}-01 \\
9.847 \mathrm{E}-01 \\
9.841 \mathrm{E}-01 \\
9.835 \mathrm{E}-01\end{array}$ \\
\hline $\begin{array}{l}5.000 \mathrm{E}-\mathrm{.52} \\
6.000 \mathrm{E}-02 \\
7.000 \mathrm{E}-02 \\
8.000 \mathrm{E}-02 \\
9.000 \mathrm{E}-02\end{array}$ & $\begin{array}{l}9.830 \mathrm{E}-01 \\
9.824 \mathrm{E}-01 \\
9.819 \mathrm{E}-01 \\
9.813 \mathrm{E}-01 \\
9.807 \mathrm{E}-01\end{array}$ & $\begin{array}{l}9.830 \mathrm{E}-01 \\
9.824 \mathrm{E}-01 \\
9.819 \mathrm{E}-01 \\
9.813 \mathrm{E}-01 \\
9.807 \mathrm{E}-01\end{array}$ & $\begin{array}{l}9.830 E-01 \\
9.824 E-01 \\
9.819 E-01 \\
9.813 E-01 \\
9.807 E-01\end{array}$ \\
\hline $\begin{array}{l}1.000 E-01 \\
1.500 E-01 \\
2.000 E-01 \\
3.000 E-01 \\
4.000 E-01\end{array}$ & $\begin{array}{l}9.802 E-01 \\
9.774 E-01 \\
9.745 E-01 \\
9.689 E-01 \\
9.633 E-01\end{array}$ & $\begin{array}{l}9.802 E-01 \\
9.774 E-01 \\
9.745 E-01 \\
9.689 E-01 \\
9.633 E-01\end{array}$ & $\begin{array}{l}9.802 E-01 \\
9.774 E-01 \\
9.745 E-01 \\
9.689 E-01 \\
9.633 E-01\end{array}$ \\
\hline $\begin{array}{l}5.000 E-01 \\
6.000 E-01 \\
7.000 E-01 \\
8.000 E-01 \\
9.000 E-01\end{array}$ & $\begin{array}{l}9.577 E-01 \\
9.521 E-01 \\
9.464 E-01 \\
9.408 E-01 \\
9.352 E-01\end{array}$ & $\begin{array}{l}9.577 E-01 \\
9.521 E-01 \\
9.464 E-01 \\
9.408 E-01 \\
9.352 E-01\end{array}$ & $\begin{array}{l}9.577 E-01 \\
9.521 E-01 \\
9.464 E-01 \\
9.408 E-01 \\
9.352 E-01\end{array}$ \\
\hline $\begin{array}{l}1.000 \mathrm{E}+00 \\
1.500 \mathrm{E}+00 \\
2.000 \mathrm{E}+00 \\
3.000 \mathrm{E}+00 \\
4.000 \mathrm{E}+00\end{array}$ & $\begin{array}{l}9.296 \mathrm{E}-01 \\
9.016 \mathrm{E}-01 \\
8.738 \mathrm{E}-01 \\
8.185 \mathrm{E}-01 \\
7.642 \mathrm{E}-01\end{array}$ & $\begin{array}{l}9.296 \mathrm{E}-01 \\
9.016 \mathrm{E}-01 \\
8.738 \mathrm{E}-01 \\
8,185 \mathrm{E}-01 \\
7.642 \mathrm{E}-01\end{array}$ & $\begin{array}{l}9.296 E-01 \\
9.016 E-01 \\
8.738 E-01 \\
8.185 E-01 \\
7.642 E-01\end{array}$ \\
\hline $\begin{array}{l}5.000 \mathrm{E}+00 \\
6.000 \mathrm{E}+00 \\
7.000 \mathrm{E}+00 \\
8.000 \mathrm{E}+00 \\
9.000 \mathrm{E}+00\end{array}$ & $\begin{array}{l}7.109 \mathrm{E}-01 \\
6.591 \mathrm{E}-01 \\
6.088 \mathrm{E}-01 \\
5.603 \mathrm{E}-01 \\
5.137 \mathrm{E}-01\end{array}$ & $\begin{array}{l}7.109 E-01 \\
6.591 E-01 \\
6.088 E-01 \\
5.603 E-01 \\
5.137 E-01\end{array}$ & $\begin{array}{l}7.109 E-01 \\
6.591 E-01 \\
6.088 E-01 \\
5.603 E-01 \\
5.137 E-01\end{array}$ \\
\hline $\begin{array}{l}1.000 \mathrm{E}+01 \\
1.500 \mathrm{E}+01 \\
2.000 \mathrm{E}+01 \\
3.000 \mathrm{E}+01 \\
4.000 \mathrm{E}+01\end{array}$ & $\begin{array}{l}4.692 \mathrm{E}-01 \\
2.815 \mathrm{E}-01 \\
1.526 \mathrm{E}-01 \\
3.256 \mathrm{E}-02 \\
4.446 \mathrm{E}-03\end{array}$ & $\begin{array}{l}4.692 \mathrm{E}-01 \\
2.815 \mathrm{E}-01 \\
1.526 \mathrm{E}-01 \\
3.256 \mathrm{E}-02 \\
4.446 \mathrm{E}-03\end{array}$ & $\begin{array}{l}4.692 \mathrm{E}-01 \\
2.815 \mathrm{E}-01 \\
1.526 \mathrm{E}-01 \\
3.256 \mathrm{E}-02 \\
4.446 \mathrm{E}-03\end{array}$ \\
\hline
\end{tabular}


Table 2-12. Continued.

\begin{tabular}{cccc}
\hline $\begin{array}{c}\text { Distance } \\
\mathbf{x}(\mathrm{m})\end{array}$ & FRACVAL & STEFH* $^{*}$ & FRACFLO \\
\hline $5.000 \mathrm{E}+01$ & $3.824 \mathrm{E}-04$ & $3.824 \mathrm{E}-04$ & $3.824 \mathrm{E}-04$ \\
$6.000 \mathrm{E}+01$ & $2.051 \mathrm{E}-05$ & $2.051 \mathrm{E}-05$ & $2.051 \mathrm{E}-05$ \\
$7.000 \mathrm{E}+01$ & $6.812 \mathrm{E}-07$ & $6.812 \mathrm{E}-07$ & $6.812 \mathrm{E}-07$ \\
$8.000 \mathrm{E}+01$ & $1.395 \mathrm{E}-08$ & $1.395 \mathrm{E}-08$ & $1.395 \mathrm{E}-08$ \\
$9.000 \mathrm{E}+01$ & $1.754 \mathrm{E}-10$ & $1.755 \mathrm{E}-10$ & $1.754 \mathrm{E}-10$ \\
$1.000 \mathrm{E}+02$ & $1.352 \mathrm{E}-12$ & $1.244 \mathrm{E}-12$ & $1.352 \mathrm{E}-12$ \\
\hline
\end{tabular}

- Based on Stefhest, 1970 
Table 2-13. Case 2 Results: Concentration of Np-237 in the Rock Matrix, at Time $t=10,000$ Years, Step Release Mode, Retardation Factor R' $=100$

\begin{tabular}{cccc}
\hline \hline $\begin{array}{c}\text { Distance } \\
\text { x }(\mathrm{m})\end{array}$ & FRACVAL & STEFH* & FRACFLO \\
\hline $1.000 \mathrm{E}-02$ & $8.818 \mathrm{E}-01$ & $8.818 \mathrm{E}-01$ & $8.818 \mathrm{E}-01$ \\
$1.500 \mathrm{E}-02$ & $8.790 \mathrm{E}-01$ & $8.790 \mathrm{E}-01$ & $8.790 \mathrm{E}-01$ \\
$2.000 \mathrm{E}-02$ & $8.763 \mathrm{E}-01$ & $8.763 \mathrm{E}-01$ & $8.763 \mathrm{E}-01$ \\
$3.000 \mathrm{E}-02$ & $8.707 \mathrm{E}-01$ & $8.707 \mathrm{E}-01$ & $8.707 \mathrm{E}-01$ \\
$4.000 \mathrm{E}-02$ & $8.652 \mathrm{E}-01$ & $8.652 \mathrm{E}-01$ & $8.652 \mathrm{E}-01$ \\
& & & \\
$5.000 \mathrm{E}-02$ & $8.596 \mathrm{E}-01$ & $8.596 \mathrm{E}-01$ & $8.596 \mathrm{E}-01$ \\
$6.000 \mathrm{E}-02$ & $8.541 \mathrm{E}-01$ & $8.541 \mathrm{E}-01$ & $8.541 \mathrm{E}-01$ \\
$7.000 \mathrm{E}-02$ & $8.485 \mathrm{E}-01$ & $8.485 \mathrm{E}-01$ & $8.485 \mathrm{E}-01$ \\
$8.000 \mathrm{E}-02$ & $8.430 \mathrm{E}-01$ & $8.430 \mathrm{E}-01$ & $8.430 \mathrm{E}-01$ \\
$9.000 \mathrm{E}-02$ & $8.375 \mathrm{E}-01$ & $8.375 \mathrm{E}-01$ & $8.375 \mathrm{E}-01$ \\
& & & \\
$1.000 \mathrm{E}-01$ & $8.320 \mathrm{E}-01$ & $8.320 \mathrm{E}-01$ & $8.320 \mathrm{E}-01$ \\
$1.500 \mathrm{E}-01$ & $8.046 \mathrm{E}-01$ & $8.046 \mathrm{E}-01$ & $8.046 \mathrm{E}-01$ \\
$2.000 \mathrm{E}-01$ & $7.774 \mathrm{E}-01$ & $7.774 \mathrm{E}-01$ & $7.774 \mathrm{E}-01$ \\
$3.000 \mathrm{E}-01$ & $7.239 \mathrm{E}-01$ & $7.239 \mathrm{E}-01$ & $7.239 \mathrm{E}-01$ \\
$4.000 \mathrm{E}-01$ & $6.716 \mathrm{E}-01$ & $6.716 \mathrm{E}-01$ & $6.716 \mathrm{E}-01$ \\
& & & \\
$5.000 \mathrm{E}-01$ & $6.209 \mathrm{E}-01$ & $6.209 \mathrm{E}-01$ & $6.209 \mathrm{E}-01$ \\
$6.000 \mathrm{E}-01$ & $5.720 \mathrm{E}-01$ & $5.720 \mathrm{E}-01$ & $5.720 \mathrm{E}-01$ \\
$7.000 \mathrm{E}-01$ & $5.249 \mathrm{E}-01$ & $5.249 \mathrm{E}-01$ & $5.249 \mathrm{E}-01$ \\
$8.000 \mathrm{E}-01$ & $4.799 \mathrm{E}-01$ & $4.799 \mathrm{E}-01$ & $4.799 \mathrm{E}-01$ \\
$9.000 \mathrm{E}-01$ & $4.371 \mathrm{E}-01$ & $4.371 \mathrm{E}-01$ & $4.371 \mathrm{E}-01$ \\
& & & \\
$1.000 \mathrm{E}+00$ & $3.966 \mathrm{E}-01$ & $3.966 \mathrm{E}-01$ & $3.966 \mathrm{E}-01$ \\
$1.500 \mathrm{E}+00$ & $2.297 \mathrm{E}-01$ & $2.297 \mathrm{E}-01$ & $2.297 \mathrm{E}-01$ \\
$2.000 \mathrm{E}+00$ & $1.201 \mathrm{E}-01$ & $1.201 \mathrm{E}-01$. & $1.201 \mathrm{E}-01$ \\
$3.000 \mathrm{E}+00$ & $2.373 \mathrm{E}-02$ & $2.373 \mathrm{E}-02$ & $2.373 \mathrm{E}-02$ \\
$4.000 \mathrm{E}+00$ & $2.990 \mathrm{E}-03$ & $2.990 \mathrm{E}-0.3$ & $2.990 \mathrm{E}-03$ \\
& & & \\
$5.000 \mathrm{E}+00$ & $2.369 \mathrm{E}-04$ & $2.369 \mathrm{E}-04$ & $2.369 \mathrm{E}-04$ \\
$6.000 \mathrm{E}+00$ & $1.169 \mathrm{E}-05$ & $1.169 \mathrm{E}-05$ & $1.169 \mathrm{E}-05$ \\
$7.000 \mathrm{E}+00$ & $3.568 \mathrm{E}-07$ & $3.568 \mathrm{E}-07$ & $3.568 \mathrm{E}-07$ \\
$8.000 \mathrm{E}+00$ & $6.709 \mathrm{E}-09$ & $6.710 \mathrm{E}-09$ & $6.709 \mathrm{E}-09$ \\
$9.000 \mathrm{E}+00$ & $7.746 \mathrm{E}-11$ & $7.725 \mathrm{E}-11$ & $7.746 \mathrm{E}-11$ \\
& & & \\
$1.000 \mathrm{E}+01$ & $5.479 \mathrm{E}-13$ & $5.371 \mathrm{E}-13$ & $5.479 \mathrm{E}-13$ \\
\hline \hline
\end{tabular}

* Based on Stefhest, 1970 
Table 2-14. Case 2 Results: Concentration of Np-237 in the Rock Matrix, at Time $t=10,000$ Years, Step

Release Mode, Retardation Factor $R^{\prime}=10,000$

\begin{tabular}{cccc}
\hline $\begin{array}{c}\text { Distance } \\
\mathbf{x}(\mathrm{m})\end{array}$ & FRACVAL & STEFH" & FRACFLO \\
\hline $1.000 \mathrm{E}-02$ & $1.473 \mathrm{E}-01$ & $1.473 \mathrm{E}-01$ & $1.473 \mathrm{E}-01$ \\
$1.500 \mathrm{E}-02$ & $1.377 \mathrm{E}-01$ & $1.377 \mathrm{E}-01$ & $1.377 \mathrm{E}-01$ \\
$2.000 \mathrm{E}-02$ & $1.286 \mathrm{E}-01$ & $1.286 \mathrm{E}-01$ & $1.286 \mathrm{E}-01$ \\
$3.000 \mathrm{E}-02$ & $1.118 \mathrm{E}-01$ & $1.118 \mathrm{E}-01$ & $1.118 \mathrm{E}-01$ \\
$4.000 \mathrm{E}-02$ & $9.676 \mathrm{E}-02$ & $9.676 \mathrm{E}-0.2$ & $9.676 \mathrm{E}-02$ \\
& & & \\
$5.000 \mathrm{E}-02$ & $8.339 \mathrm{E}-02$ & $8.339 \mathrm{E}-02$ & $8.339 \mathrm{E}-02$ \\
$6.000 \mathrm{E}-02$ & $7.156 \mathrm{E}-02$ & $7.156 \mathrm{E}-02$ & $7.156 \mathrm{E}-02$ \\
$7.000 \mathrm{E}-02$ & $6.114 \mathrm{E}-02$ & $6.114 \mathrm{E}-02$ & $6.114 \mathrm{E}-02$ \\
$8.000 \mathrm{E}-02$ & $5.201 \mathrm{E}-02$ & $5.201 \mathrm{E}-02$ & $5.201 \mathrm{E}-02$ \\
$9.000 \mathrm{E}-02$ & $4.405 \mathrm{E}-02$ & $4.405 \mathrm{E}-02$ & $4.405 \mathrm{E}-02$ \\
& & & \\
$1.000 \mathrm{E}-01$ & $3.714 \mathrm{E}-02$ & $3.714 \mathrm{E}-02$ & $3.714 \mathrm{E}-02$ \\
$1.500 \mathrm{E}-01$ & $1.481 \mathrm{E}-02$ & $1.481 \mathrm{E}-02$ & $1.481 \mathrm{E}-0.2$ \\
$2.000 \mathrm{E}-01$ & $5.271 \mathrm{E}-03$ & $5.271 \mathrm{E}-03$ & $5.271 \mathrm{E}-03$ \\
$3.000 \mathrm{E}-01$ & $4.726 \mathrm{E}-04$ & $4.726 \mathrm{E}-04$ & $4.726 \mathrm{E}-04$ \\
$4.000 \mathrm{E}-01$ & $2.648 \mathrm{E}-0.5$ & $2.648 \mathrm{E}-05$ & $2.648 \mathrm{E}-0.5$ \\
& & & \\
$5.000 \mathrm{E}-01$ & $9.212 \mathrm{E}-07$ & $9.212 \mathrm{E}-07$ & $9.212 \mathrm{E}-07$ \\
$6.000 \mathrm{E}-01$ & $1.986 \mathrm{E}-08$ & $1.980 \mathrm{E}-08$ & $1.980 \mathrm{E}-08$ \\
$7.000 \mathrm{E}-01$ & $2.619 \mathrm{E}-10$ & $2.622 \mathrm{E}-10$ & $2.619 \mathrm{E}-10$ \\
$8.000 \mathrm{E}-01$ & $2.128 \mathrm{E}-12$ & $1.973 \mathrm{E}-12$ & $2.128 \mathrm{E}-12$ \\
$9.000 \mathrm{E}-01$ & $1.060 \mathrm{E}-14$ & $7.621 \mathrm{E}-14$ & $1.060 \mathrm{E}-14$ \\
& & & \\
$1.000 \mathrm{E}+00$ & $3.230 \mathrm{E}-17$ & $-1.697 \mathrm{E}-14$ & $3.230 \mathrm{E}-17$ \\
\hline
\end{tabular}

* Based on Stefhest, 1970 


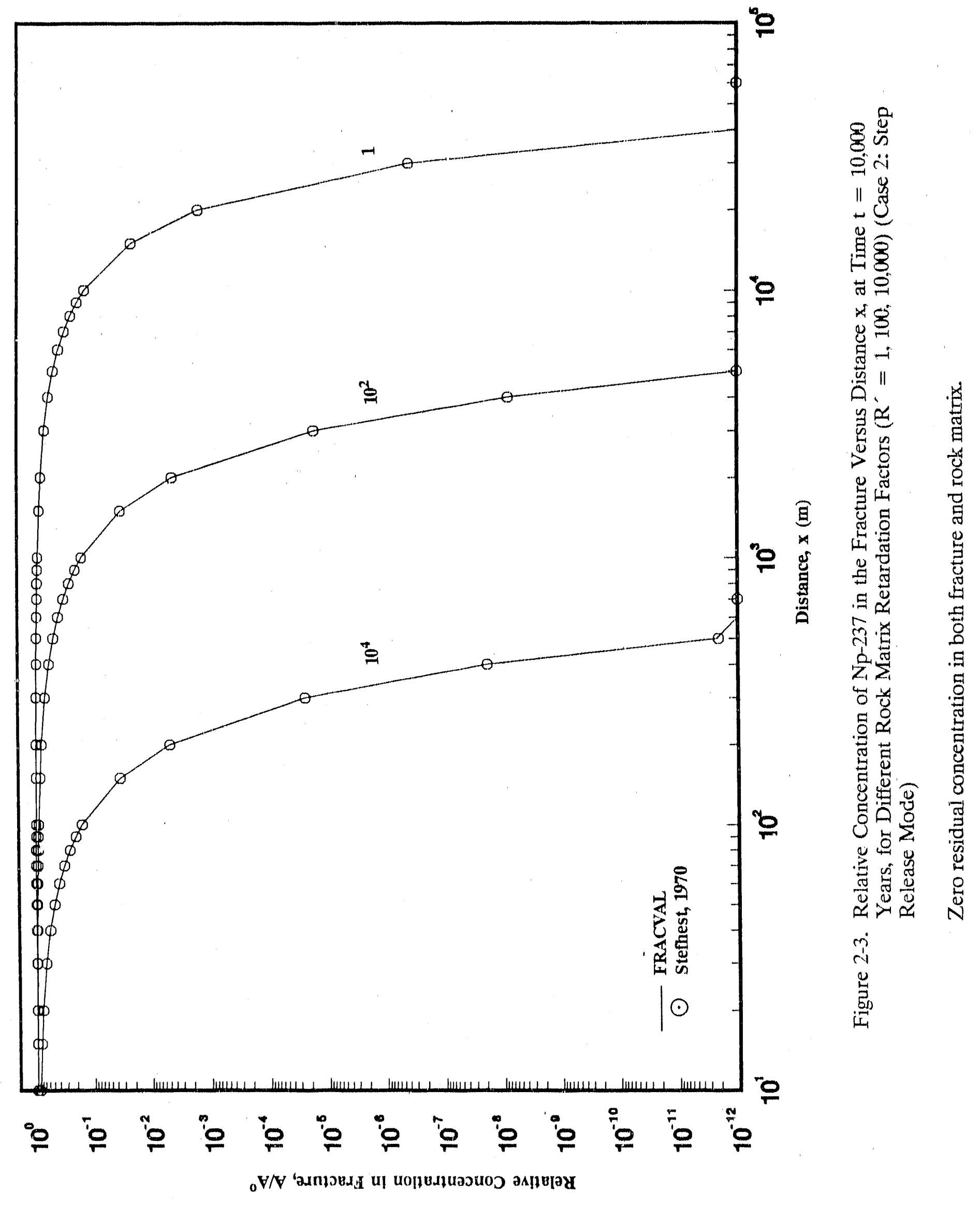




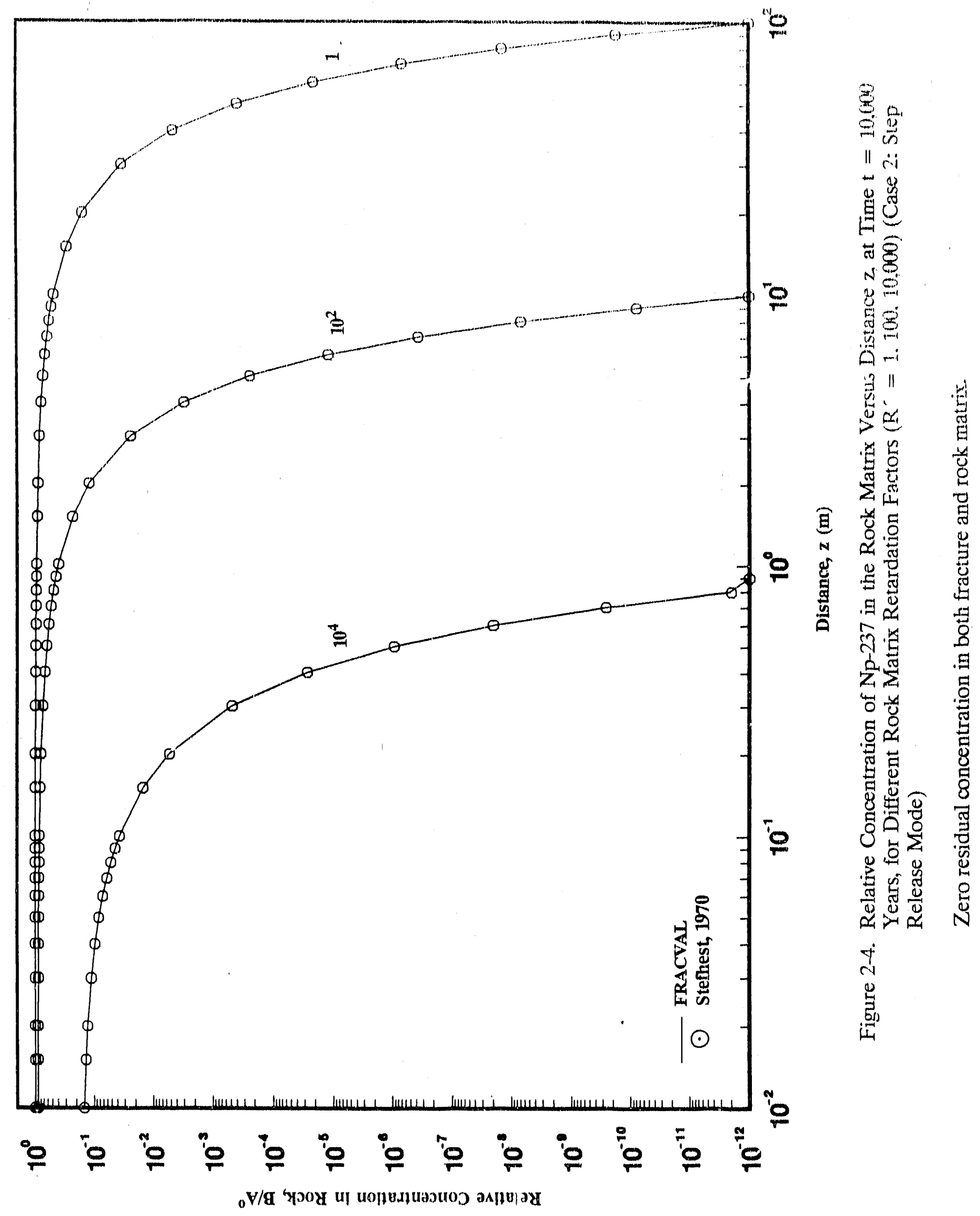




\section{2 .3 Cano 3 Rosults}

Case 3 oxaminos the concontration of $\mathrm{Np}-237$ for a non-zoro rostdual concontralion distribution

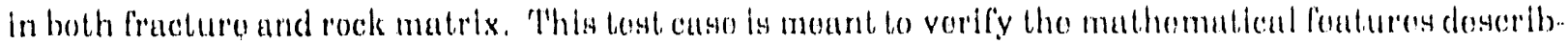

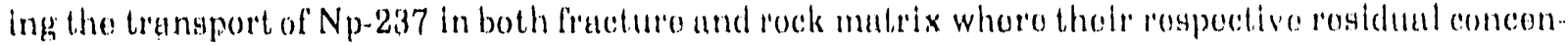

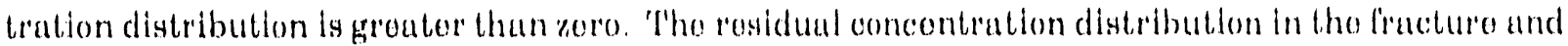
rock matrix at timo oquals aoro is assumod to be non-uniform in tho case of tho first modium (soo Ficuation 2-7) and uniform (seo licpuation 2 8) in the case of the second modium. Solute rolonsod at the

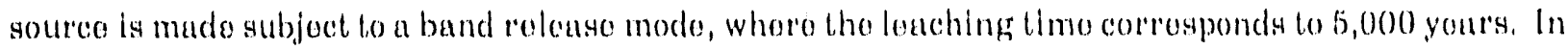
the simulations reported horo, throe values of the rock matrix rotardation factor woro investigatod. Based on tho data prosentod in T'able 2-15, the numorical rosults roportod in Tables 2-16 through 2-18 (fracturo) und T'ables $2-19$ through $2-21$ (rock matrix) show that both solutions WRACVAL and S'l'LIH aro in excollent agreoment.

Figures 2-5 and 2-6 show the temporal rolative concontration of $\mathrm{Np} 237$ obsorved in tho fracture at a distance of $100 \mathrm{~m}$ downstrea from the source and the spatial distribution in tho rock mulrix observed at lime corresponding to 10,000 yoars. 'Threo simulations wero performed corresponding to three values of the rock rolardation finctor $\left(R^{\prime}=1,100\right.$, and 10,000$)$.

\subsubsection{Cano 4 Results}

Caso 4 examines the influence of the hydrodynamic disporsion coefficients on the spatial behavior of $\mathrm{Np}-237$ in both fracture and rock matrix. In the course of these investigations, the disporsionfreosolution as reported in Section 2.1 was also vorifled. As in the fornor tost case, a non zero rosidual concentration is assumed, with a non-uniform distribution in the fracture and uniform in the rock matrix. Solute relouse from the source is subject to a band roloase mode, where the loaching time corresponds 601,000 yoars. In these simulations fivo values of the hydrodynamic disporsion wero investigated. Based on tho data prosented in 'Table 2.22, the numorical results reported in Tables 2.23 through 2227 (fracture) and Tables 2 -28 through 2-32 (rock matrix) show that, both solutions FRACVAL and S'I'LFH aro in excellont agroomont.

Figures 2.7 and 2.8 show the spatial relative concontration ol $\mathrm{Np}$-2.37 obsorvod in the fracture and in the rock matrix at a distance of 100 m downstroam from tho sourco for a simulation timo corresponding to 5,000 yoar's. 'The influonce of the hydrodynamic dispersion coofliciont bocomes noticouble when it exceeds the value of $100 \mathrm{~m} 2 / \mathrm{yr}$. The concontration in the fracture far downstres on from tho source registers a drop from its original value of approximatedy $1.5 \times 10^{-4}$ down to a value of $1.051 \mathrm{x}$ $10^{-5}$ (see figuro 2-7). 'I'his confirms theory, which for the choice of solected parmoler values reluted to the residual concontration distribution in both media, suggests that in tho absence of solute roleased 
'T'ablo 2-15. Input Paramalors for' Caso is

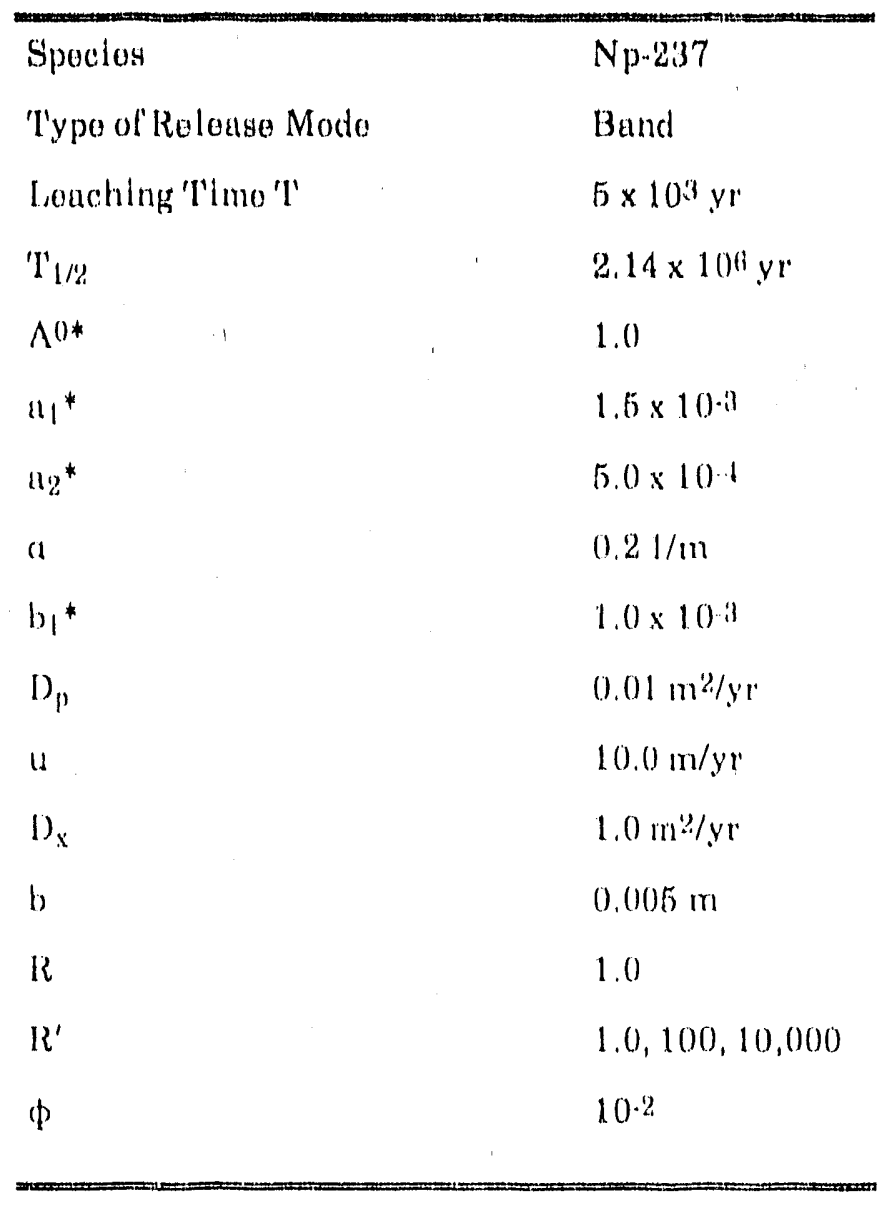

*(arbitrary unlts of activity/L,3) 
T'able 2.16, Case 3 Rosults: Concentration of $\mathrm{Np}-237$ in the Fracture, at $\mathrm{x}=100 \mathrm{~m}$, Bund Rolouso Mode, and Rotardation Factor $R^{\prime}=1$

\begin{tabular}{|c|c|c|c|c|c|}
\hline $\begin{array}{l}\text { Tlme } \\
t(y r)\end{array}$ & FRACVAL & STEFH" & $\begin{array}{l}\text { Tlimo } \\
t\left(y r^{\prime}\right)\end{array}$ & FRACVAL & S'IEHI' \\
\hline $1,000 \mathrm{E}+00$ & $1.405 \mathrm{E}-0.3$ & $1.405 E-03$ & $5.000 \mathrm{E}+0.3$ & $9,82,4 \mathrm{E}-01$ & $9.82 .5 \mathrm{E}-0.1$ \\
\hline $1.500 \mathrm{E}+00$ & $1.387 \mathrm{E}-03$ & $1,387 \mathrm{E}-0.3$ & $6.0(0) \mathrm{E}+03$ & $2.124 \mathrm{E}-02$ & $2.12 .51-(12$ \\
\hline $2.000 \mathrm{E}+00$ & $1.373 E-03$ & $1.373 \mathrm{E}-0.3$ & $7.0 \times 0012+0.3$ & $1.178 \mathrm{E}-(12$ & $1.178 \mathrm{E}-(12$ \\
\hline $3,000 \mathrm{E}+100$ & $1.3 .52 \mathrm{E}-03$ & $1.356 \mathrm{E}-03$ & $8,0000 E+03$ & $8.001 \mathrm{E}-03$ & $8,0(02 \mathrm{E}-0) 3$ \\
\hline $4,(00) E+00$ & $1.335 E-03$ & $1.403 \mathrm{E}-03$ & $9.000 \mathrm{E}+03$ & $5.955 \mathrm{E}-\cdots(13$ & $5.456 \mathrm{E}-(1) 3$ \\
\hline $5.000 \mathrm{E}+00$ & $1.322 E-03$ & $1.057 \mathrm{E}-03$ & $1.000 \mathrm{E}+04$ & $4.679 \mathrm{E}-03$ & $4.680 \mathrm{E}-(0.3$ \\
\hline $6.000 \mathrm{E}+00$ & $1.310 \mathrm{E}-03$ & $1.450 \mathrm{E}-03$ & $1.500 \mathrm{E}+04$ & $2.071 E \ldots 03$ & $2.072 \mathrm{E}-0.3$ \\
\hline $7.000 \mathrm{E}+00$ & $1.301 E-03$ & $7,417 E-04$ & $2.000 \mathrm{E}+0.4$ & $1.235 \mathrm{E}-0.3$ & $1.235 E-03$ \\
\hline $8.000 \mathrm{E}+00$ & $1.293 \mathrm{E}-03$ & $4.895 E-03$ & $3,000 \mathrm{E}+04$ & $6.220 \mathrm{E}-04$ & $6.226 \mathrm{E}-(04$ \\
\hline $9.000 \mathrm{E}+00$ & $1.369 \mathrm{E}-03$ & $-8.316 \mathrm{E}-03$ & $4.0000 \mathrm{E}+04$ & $3,898 \mathrm{E}-04$ & $3.902 \mathrm{E}-04$ \\
\hline $1.000 \mathrm{E}+01$ & $2.109 E-02$ & $3,294 \mathrm{E}-02$ & $5.000 \mathrm{E}+04$ & $2.732 E-04$ & $2.736 \mathrm{E}-04$ \\
\hline $1.500 \mathrm{E}+01$ & $5.262 \mathrm{E}-01$ & $5.276 \mathrm{E}-01$ & $6.000 \mathrm{E}+04$ & $2.051 \mathrm{E}-04$ & $2.055 E-04$ \\
\hline $2.000 \mathrm{E}+01$ & $6.546 \mathrm{E}-01$ & $6.555 \mathrm{E}-01$ & $7.000 \mathrm{E}+04$ & $1.612 E-04$ & $1.616 \mathrm{E}-04$ \\
\hline $3.000 \mathrm{E}+01$ & $7.519 \mathrm{E}-0.1$ & $7.519 \mathrm{E}-01$ & $8.0001 \mathrm{E}+04$ & $1.311 E-04$ & $1.314 E-04$ \\
\hline $4.000 \mathrm{E}+01$ & $7.964 \mathrm{E}-01$ & $7.964 \mathrm{E}-01$ & $9.000 \mathrm{E}+04$ & $1.093 \mathrm{E}-04$ & $1.096 \mathrm{E}-04$ \\
\hline $5.000 \mathrm{E}+01$ & $8.232 E-01$ & $8.232 \mathrm{E}-01$ & $1.0000 \mathrm{E}+05$ & $9,291 \mathrm{E}-0.5$ & $9.321 \mathrm{E}-0.5$ \\
\hline $6.000 \mathrm{E}+01$ & $8.416 \mathrm{E}-01$ & $8.416 \mathrm{E}-01$ & $1.500 \mathrm{E}+05$ & $4.998 E-05$ & $5.022 \mathrm{E}-0.5$ \\
\hline $7.000 \mathrm{E}+01$ & $8.552 \mathrm{E}-01$ & $8.552 \mathrm{E}-01$ & $2.0000 E+05$ & $3.229 \mathrm{E}-05$ & $3.249 \mathrm{E}-\cdots 0.5$ \\
\hline $8.000 \mathrm{E}+01$ & $8.659 \mathrm{E}-01$ & $8.659 \mathrm{E}-01$ & $3.000 \mathrm{E}+05$ & $1.748 E-05$ & $1.765 \mathrm{E}-0.5$ \\
\hline $9.000 \mathrm{E}+01$ & $8.744 \mathrm{E}-01$ & $8.745 \mathrm{E}-01$ & $4.000 \mathrm{E}+05$ & $1.132 E-05$ & $1.1 .46 \mathrm{E}-0.5$ \\
\hline $1.000 E+02$ & $8.816 \mathrm{E}-01$ & $8.816 \mathrm{E}-01$ & $5.000 \mathrm{E}+05$ & $8.076 \mathrm{E} \cdots 06$ & $8.194 E-06$ \\
\hline $1.500 \mathrm{E}+02$ & $9.049 \mathrm{E}-01$ & $9.049 \mathrm{E}-01$ & $6.000 \mathrm{E}+05$ & $6.124 \mathrm{E}-06$ & $6.228 \mathrm{E}-06$ \\
\hline $2.000 \mathrm{E}+02$ & $9.183 \mathrm{E}-01$ & $9.183 \mathrm{E}-0.1$ & $7.000 \mathrm{E}+05$ & $4.842 \mathrm{E}-06$ & $4.935 \mathrm{E}-06$ \\
\hline $3.000 E+02$ & $9.338 E-01$ & $9.338 E--01$ & $8.000 E+0.5$ & $3.945 E-06$ & $4.031 E-06$ \\
\hline $4.000 \mathrm{E}+02$ & $9.428 \mathrm{E}-01$ & $428 \mathrm{E}-01$ & $9.000 \mathrm{E}+05$ & $3.290 E-06$ & $3.368 \mathrm{E}-06$ \\
\hline $5.000 \mathrm{E}+02$ & $9.490 \mathrm{E}-01$ & $9.490 \mathrm{E}-01$ & 1.00NDE + 06 & $2.793 \mathrm{E}-06$ & $2.864 \mathrm{E}-06$ \\
\hline $6.000 \mathrm{E}+02$ & $9.534 \mathrm{E}-01$ & $9.534 \mathrm{E}-01$ & & & \\
\hline $7.000 \mathrm{E}+02$ & $9.569 E-01$ & $9.569 \mathrm{E}-01$ & & & \\
\hline $8.000 \mathrm{E}+02$ & $9.597 E-01$ & $9.597 \mathrm{E}-01$ & & & \\
\hline $9.000 \mathrm{E}+02$ & $9.619 \mathrm{E}-01$ & $9.619 \mathrm{E}-01$ & & & \\
\hline $1.000 \mathrm{E}+03$ & $9.639 \mathrm{E}-01$ & $9.639 \mathrm{E}-01$ & & & \\
\hline $1.500 \mathrm{E}+03$ & $9.703 E-01$ & $9.703 \mathrm{E}-01$ & & & \\
\hline $2.000 \mathrm{E}+03$ & $9.741 E-0.1$ & $9.7 / 41 E-01$ & & & \\
\hline $3.000 E+03$ & $9.784 \mathrm{E}-01$ & $9.784 \mathrm{E}-01$ & & & \\
\hline $4.000 \mathrm{E}+03$ & $9.809 E-01$ & $9.809 \mathrm{E}-01$ & & & \\
\hline
\end{tabular}

* Based on Stefhest, 1970) 
Tablo 2.17. Caso 3 Results: Concontration of Np-2377 in tho Fracture, at $x=100 \mathrm{~m}$, Bund Roloase Modo, and Roturdation Factor $\mathbb{R}^{\prime}=10$ 1.X)

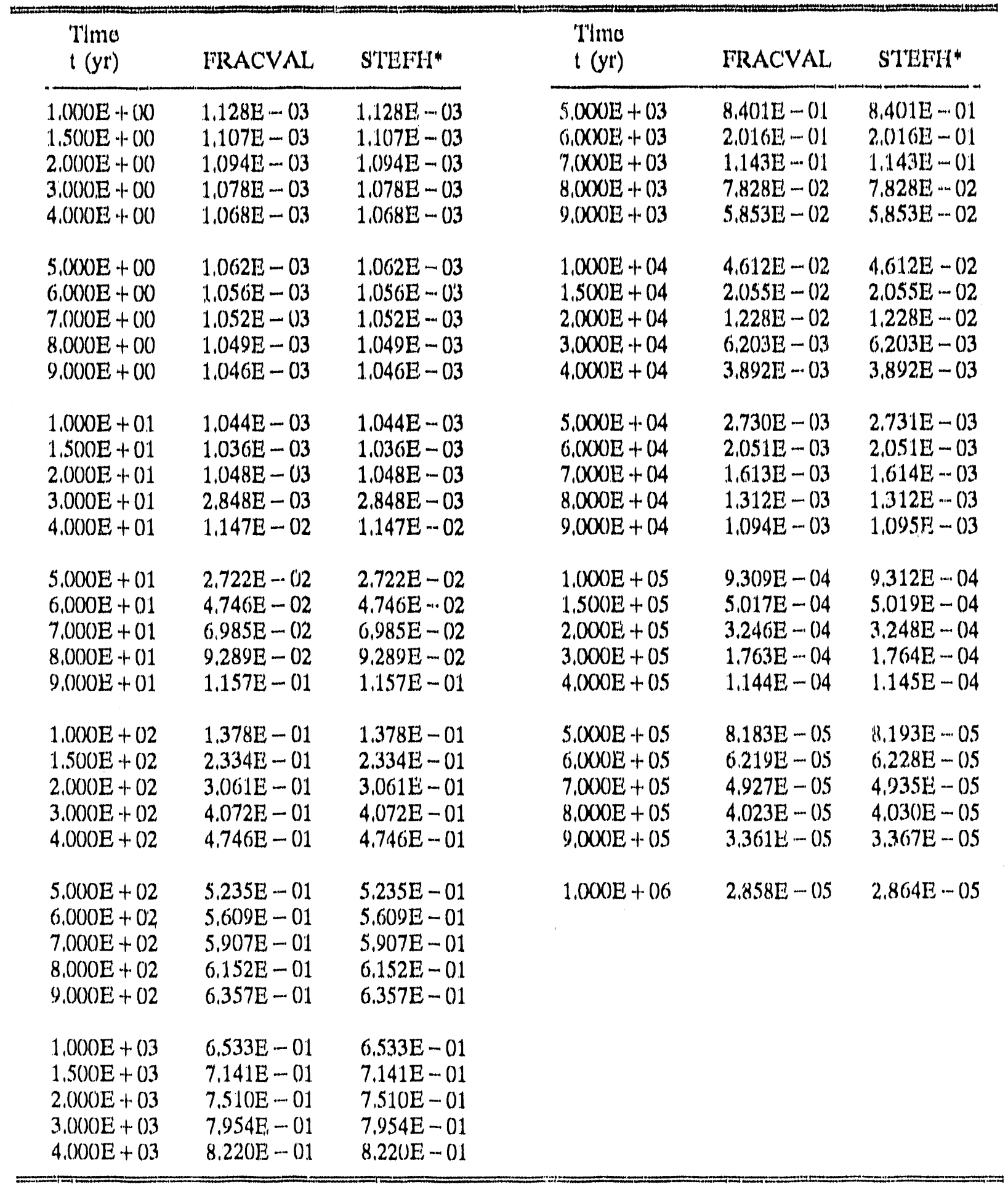

* Based on Stefhest, 1970 


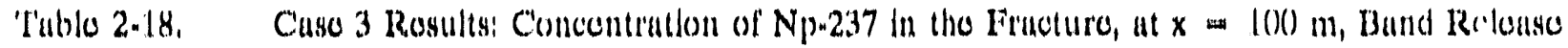
Modo, and Roturdation F'actor $R^{\prime}$ ta $10,(X X)$

\begin{tabular}{|c|c|c|c|c|c|}
\hline $\begin{array}{l}\text { I'Imo } \\
l\left(y t^{\prime}\right)\end{array}$ & FRACVAL. & S'IEFH" & $\begin{array}{l}\text { I'lmo } \\
t\left(y r^{\prime}\right)\end{array}$ & FRACVAL & S'LEH' \\
\hline $1,(0)(0) \mathrm{E}+0(0)$ & $1.014 E-0.3$ & $1,014 \mathrm{E}-0.3$ & $5 .(X) X E+0) 3$ & $4,7(12 \mathrm{E}-(12$ & $4.7(1212-\cdots(02$ \\
\hline $1.5(x) \mathrm{E} ?+00$ & $1.012 \mathrm{E}-\cdots, 03$ & $1,(012 \mathrm{E}-103$ & $(i,(X)(X) E+0.3$ & $6,9361 \mathrm{-1}-12$ & $(0,936 \mathrm{E}-\cdots(12$ \\
\hline $2.00(0)+00$ & $1.010 \mathrm{E}-03$ & $1.010 \mathrm{E}-03$ & $7,(X X) E+0) 3$ & $9.0671-02$ & $9,(0,57 \mathrm{E},-12$ \\
\hline $3,(0)(0) E+0)$ & $1.008 \mathrm{E}-0.3$ & $1,008 \mathrm{E}-0.3$ & $8 .(X) 1)+(1) 3$ & $1,04913,-0.1$ & $1,040 \mathrm{E}-0.01$ \\
\hline $4,000 \mathrm{E}+0(0)$ & $1.007 \mathrm{E}-03$ & $1,007 \mathrm{E}-0.3$ & $9 .(0)(X) E+03$ & $1.1131, \cdots(01$ & $1,113 E-(01$ \\
\hline $5.00013+00$ & $1.006 \mathrm{E}-03$ & $1,006 \mathrm{E}-0.3$ & $1.000) \mathrm{E}_{2}+04$ & $1,123 \mathrm{E}-01$ & $1.123 \mathrm{E}-01$ \\
\hline $6,000 \mathrm{E}+00$ & $1,006 \mathrm{E}-03$ & $1.006 \mathrm{E}-03$ & $1.50(0) \mathrm{E}+0.4$ & $9.107 \mathrm{E}-02$ & $9,107 \mathrm{E}-(12$ \\
\hline $7.000 \mathrm{E}+00$ & $1,005 \mathrm{E}-03$ & $1 .(00.5 \mathrm{E}-0.03$ & $2.000 \mathrm{E}+04$ & $(0,922 \mathrm{E}-(12$ & $(0.922 \mathrm{E}-02$ \\
\hline $8,000 \mathrm{E}+00$ & $1,0(0.5 \mathrm{E}-03$ & $1,00.5 \mathrm{E}-0.3$ & $3.000 \mathrm{E}+04$ & $4.323 \mathrm{E}-02$ & $4.323 \mathrm{E}-02$ \\
\hline $9.000 \mathrm{E}+00$ & $1.005 \mathrm{EE}-0.03$ & $1,005 \mathrm{E}-03$ & $4.000 \mathrm{E}+04$ & $2.991 \mathrm{E}-02$ & $2.991 \mathrm{E} \quad 02$ \\
\hline $1.000 \mathrm{E}+01$ & $1.004 \mathrm{E}-0.3$ & $1,004 E-0,3$ & $5.000 \mathrm{E}+0.4$ & $2.220 \mathrm{E}-02$ & $2.220 \mathrm{E}-02$ \\
\hline $1.500 \mathrm{E}+01$ & $1,004 \mathrm{~F}:-03$ & $1.004 E-03$ & $6.000 \mathrm{E}+04$ & $1,72,9 \mathrm{E} \cdots 02$ & $1,729 \mathrm{E}-0.02$ \\
\hline $2,000 \mathrm{E}+01$ & $1.003 E-03$ & $1.003 \mathrm{E}-0.3$ & $7.00(X) E+04$ & $1,396 \mathrm{E}-02$ & $1.396 E-02$ \\
\hline $3,000 \mathrm{E}+01$ & $1.003 \mathrm{E}-03$ & $1.003 E=03$ & 8.0 CX)E +04 & $1.157 \mathrm{E}-02$ & $1,157 E-02$ \\
\hline $4.000 \mathrm{E}+01$ & $1.0(12 \mathrm{E} \cdots 03$ & $1,002 \mathrm{E}-0.3$ & $9.0(0) E+0.4$ & $9.794 \mathrm{E}-03$ & $9.794 \mathrm{E}-0.3$ \\
\hline $5.000 \mathrm{E}+01$ & $1.002 \mathrm{E}-0.3$ & $1.002 \mathrm{E} \cdots 03$ & $1,0(x) E+05$ & $8,430 \mathrm{E} \cdots 03$ & $8,430 \mathrm{E} \cdots 03$ \\
\hline $6.000 \mathrm{E}+01$ & $1.002 E-03$ & $1 .(0) 2 E-03$ & $1.500 E+05$ & $4.704 \mathrm{E}-0.3$ & $4,7(04 E-0) 3$ \\
\hline $7.000 \mathrm{E}+01$ & $1.002 E-03$ & $1.002 \mathrm{E}-03$ & $2,000 \mathrm{E}+05$ & $3,096 \mathrm{E}-03$ & $3,(096 \mathrm{E}-03$ \\
\hline $8,000 \mathrm{E}+01$ & $1.002 \mathrm{E}-0.3$ & $1 .(0) 2 \mathrm{E}-03$ & $3.00(0) \mathrm{E}+0.5$ & $1.710 \mathrm{E}-03$ & $1.710 \mathrm{E}-03$ \\
\hline $9.0(0) \mathrm{E}+01$ & $1.001 \mathrm{E}-03$ & $1,001 \mathrm{E}-03$ & $4,0000 \mathrm{E}+05$ & $1.120 \mathrm{E}-03$ & $1,120 \mathrm{E},-0.3$ \\
\hline $1.000 \mathrm{E}+(02$ & $1 .(0) 1 E-03$ & $1,001 \mathrm{E}-0.3$ & $5.00(0) \mathrm{E}+0.5$ & $8.0491-04$ & $8.049 \mathrm{~A}-1-04$ \\
\hline $1.500 \mathrm{E}+02$ & $1,001 E-03$ & $1 .(0) 1 \mathrm{E}-0.3$ & $6.000 \mathrm{E}+0.5$ & $0.138 E-04$ & $6.138 \mathrm{E}-04$ \\
\hline $2.000 \mathrm{E}+012$ & $1.0(0) 1 E-03$ & $1.001 \mathrm{E}-0.3$ & $7,0000 \mathrm{E}+0.5$ & $4.875 E-04$ & $4.875 \mathrm{E},-04$ \\
\hline $3.000 \mathrm{E}+02$ & $1.001 \mathrm{E}-03$ & $1.001 \mathrm{E}-03$ & $8.000 \mathrm{E}+0.5$ & $3.988 \mathrm{E}-04$ & $3,988 \mathrm{E}-0.14$ \\
\hline $4.000 \mathrm{E}+(02$ & $1 .(0) 1 E-03$ & $1.001 E-03$ & $9.0000 \mathrm{E}+0.5$ & $3.336 \mathrm{E} \cdots(14$ & $3,337 \mathrm{E}-04$ \\
\hline $5,000 \mathrm{E}+02$ & $1.000 \mathrm{E}-03$ & $1.000 \mathrm{E}-03$ & $1.000 . E+06$ & $2.841 \mathrm{E}-04$ & $2.841 \mathrm{E}-(04$ \\
\hline $6.000 \mathrm{E}+02$ & $1.000 \mathrm{E}-03$ & $1.000 \mathrm{E}-0.03$ & $1.500 \mathrm{E}+06$ & $1.506 \mathrm{E}-0.04$ & $1.506 \mathrm{E}-04$ \\
\hline $7.000 \mathrm{E}+02$ & $1 .(000 \mathrm{E}-03$ & $1.000 \mathrm{E}-03$ & $2.0500 \mathrm{E}+06$ & $9.369 \mathrm{E}-0.5$ & $0.369 \mathrm{E}-0.5$ \\
\hline $8.0(0) \mathrm{E}+02$ & $1.001 \mathrm{E}-(1) 3$ & $1.001 E-0.3$ & $3,000 \mathrm{E}+06$ & $4.513 E \cdots 0.5$ & $4.51 .3 E-0.5$ \\
\hline $9.000 \mathrm{E}+02$ & $1.004 \mathrm{E}:-03$ & $1.004 E-03$ & $4,000 \mathrm{E}+06$ & $2.507 \mathrm{E}-0.5$ & $2.507 \mathrm{E}-0.5$ \\
\hline $1.000 \mathrm{E}+03$ & $1.011 \mathrm{E}-03$ & $1 .(011 \mathrm{E}-0.03$ & $5 .(0) 0 \mathrm{E}+06$ & $1,497 \mathrm{E} \cdots 05$ & $1.497 \mathrm{E}-0.5$ \\
\hline $1.500 \mathrm{E}+0.3$ & $1.298 \mathrm{E}-03$ & $1.298 \mathrm{E}-03$ & $6.0(x) E+06$ & $9.339 \mathrm{E}-06$ & $9.340 \mathrm{E}-06$ \\
\hline 2.000E +03 & $2.691 \mathrm{E}-03$ & $2.691 \mathrm{E}-0.3$ & 7.00()$E+06$ & $5.992 \mathrm{E}-06$ & $5.993 \mathrm{E}-06$ \\
\hline $3.000 \mathrm{E}+03$ & $1.117 E-02$ & $1.117 \mathrm{E}-02$ & $8.0000 \mathrm{E}+06$ & $3,921 \mathrm{E}-06$ & $3.921 E--06$ \\
\hline \multirow[t]{2}{*}{$4.000 \mathrm{E}+03$} & $2.683 \mathrm{E}-02$ & $2.683 \mathrm{E}-02$ & 9.0 XOOE + 06 & $2,603 \mathrm{E}-0) 6$ & $2,604 \mathrm{E}-\cdots 06$ \\
\hline & & & $1.000 \mathrm{E}+(07$ & $1.748 \mathrm{E}-106$ & $1.748 \mathrm{E}-0.0$ \\
\hline
\end{tabular}

* Based on Stefhest, 1970 
Tuble 2-19. Casc 3 Results: Concentration of Np.237 in the Rook Matrix, at $x=100 \mathrm{~m}$, Time $\mathrm{t}=10,000$ Yoars, Band Roleaso Modo, Rotardation Factor $R^{\prime}=1$

\begin{tabular}{|c|c|c|c|c|c|}
\hline $\begin{array}{c}\text { Distunco } \\
x(\mathrm{~m})\end{array}$ & FRACVAL & S'TEFH' & $\begin{array}{c}\text { Distance } \\
x(m)\end{array}$ & FRACVAL & STEFII* \\
\hline $\begin{array}{l}1.000 \mathrm{E}-02 \\
1.500 \mathrm{E}-02 \\
2.00 \mathrm{OOE}-02 \\
3 .(0 \mathrm{OONE}-02 \\
4.000 \mathrm{E}-02\end{array}$ & $\begin{array}{l}4.796 E-03 \\
4.913 E-03 \\
5.030 E-03 \\
5.263 E-13 \\
5.497 E-03\end{array}$ & $\begin{array}{l}4.796 \mathrm{E}-03 \\
4.913 \mathrm{E}-03 \\
5.030 \mathrm{E}-03 \\
5.263 \mathrm{E}-03 \\
5.497 \mathrm{E}-03\end{array}$ & $\begin{array}{l}5.000 \mathrm{E}+00 \\
6.000 \mathrm{E}+00 \\
7.000 \mathrm{E}+00 \\
8.000 \mathrm{E}+00 \\
9.000 \mathrm{E}+00\end{array}$ & $\begin{array}{l}1.101 E-01 \\
1.250 E-01 \\
1.393 E-01 \\
1.500 E-01 \\
1.580 E-01\end{array}$ & $\begin{array}{l}1.101 E-01 \\
1.259 E-01 \\
1.393 E-01 \\
1.500 E-01 \\
1.580 E-01\end{array}$ \\
\hline $\begin{array}{l}5.000 \mathrm{E}-02 \\
6.000 \mathrm{E}-02 \\
7.000 \mathrm{E}-02 \\
8.000 \mathrm{E}-02 \\
9.000 \mathrm{E}-02\end{array}$ & $\begin{array}{l}5.731 E-013 \\
5.965 E-03 \\
6.199 E-03 \\
6.433 E-03 \\
6.667 E-03\end{array}$ & $\begin{array}{l}5.731 E-03 \\
5.965 E-03 \\
6.199 E-03 \\
6.433 E-03 \\
6.667 E-03\end{array}$ & $\begin{array}{l}1.000 \mathrm{E}+01 \\
1.500 \mathrm{E}+01 \\
2.000 \mathrm{E}+01 \\
3.000 \mathrm{E}+01 \\
4.000 \mathrm{E}+01\end{array}$ & $\begin{array}{l}1.633 E-01 \\
1.543 E-01 \\
1.103 E-01 \\
3.102 E-02 \\
5.381 E-03\end{array}$ & $\begin{array}{l}1.633 E-01 \\
1.543 E-01 \\
1.103 E-01 \\
3.102 E-02 \\
5.381 E-03\end{array}$ \\
\hline $\begin{array}{l}1.000 E-01 \\
1.500 E-01 \\
2.000 E-01 \\
3.000 E-01 \\
4.000 E-01\end{array}$ & $\begin{array}{l}6.900 E-03 \\
8.069 E-03 \\
9.237 E-03 \\
1.157 E-02 \\
1.390 E-02\end{array}$ & $\begin{array}{l}6.900 E-03 \\
8.069 E-03 \\
9.237 E-03 \\
1.157 E-02 \\
1.390 E-02\end{array}$ & $\begin{array}{l}5.000 \mathrm{E}+01 \\
6.000 \mathrm{E}+01 \\
7.000 \mathrm{E}+01 \\
8.000 \mathrm{E}+01 \\
9.000 \mathrm{E}+01\end{array}$ & $\begin{array}{l}1.378 E-03 \\
1.017 E-03 \\
9.974 E-04 \\
9.968 E-04 \\
9.968 E-04\end{array}$ & $\begin{array}{l}1.378 E-03 \\
1.017 E-03 \\
9.974 E-04 \\
9.968 E-04 \\
9.968 E-04\end{array}$ \\
\hline $\begin{array}{l}5.000 \mathrm{E}-01 \\
6.000 \mathrm{E}-01 \\
7.000 \mathrm{E}-01 \\
8.000 \mathrm{E}-01 \\
9.000 \mathrm{E}-01\end{array}$ & $\begin{array}{l}1.623 E-02 \\
1.856 E-02 \\
2.088 E-02 \\
2.320 E-02 \\
2.551 E-02\end{array}$ & $\begin{array}{l}1.623 E-02 \\
1.856 E-02 \\
2.088 E-02 \\
2.320 E-02 \\
2.551 E-02\end{array}$ & $1.000 \mathrm{n} 7+02$ & $9.968 \mathrm{E}-04$ & $9.968 \mathrm{E}-04$ \\
\hline $\begin{array}{l}1.000 \mathrm{E}+00 \\
1.500 \mathrm{E}+00 \\
2.000 \mathrm{E}+00 \\
3.000 \mathrm{E}+00 \\
4.000 \mathrm{E}+00\end{array}$ & $\begin{array}{l}2.782 E-02 \\
3.925 E-02 \\
5.046 E-02 \\
7.201 E-02 \\
9.202 E-02\end{array}$ & $\begin{array}{l}2.782 \mathrm{E}-02 \\
3.925 \mathrm{E}-02 \\
5.046 \mathrm{E}-02 \\
7.201 \mathrm{E}-02 \\
9.202 \mathrm{E}-02\end{array}$ & & & \\
\hline
\end{tabular}

* Based on Stefhest, 1970 
T'uble 2420. Case 3 Results: Concentration of Npw237 in the Rock Matrix, at $x=100 \mathrm{~m}$, Time $t=10,000$ Years, Band Release Modo, Retardation Factor R' 100

\begin{tabular}{|c|c|c|c|c|c|}
\hline $\begin{array}{l}\text { Distance } \\
\mathrm{x}(\mathrm{m})\end{array}$ & FRACVAL & STEFH* & $\begin{array}{c}\text { Distance } \\
\mathrm{x}(\mathrm{m})\end{array}$ & FRACVAI & S'TEFH" $^{\prime}$ \\
\hline $\begin{array}{l}1.000 \mathrm{E}-02 \\
1.500 \mathrm{E}-02 \\
2.000 \mathrm{E}-02 \\
3.000 \mathrm{E}-02 \\
4.000 \mathrm{E}-02\end{array}$ & $\begin{array}{l}4.723 E-02 \\
4.835 E-02 \\
4.946 E-02 \\
5.168 E-02 \\
5.388 E-02\end{array}$ & $\begin{array}{l}4.723 \mathrm{E}-02 \\
4.83,5 \mathrm{E}-02 \\
4.946 \mathrm{E}-02 \\
5.168 \mathrm{E}-02 \\
5.388 \mathrm{E}-02\end{array}$ & $\begin{array}{l}1.000 \mathrm{E}+00 \\
1.500 \mathrm{E}+00 \\
2.000 \mathrm{E}+\infty 0 \\
3.000 \mathrm{E}+\infty \\
4.000 \mathrm{E}+00\end{array}$ & $\begin{array}{l}1.663 \mathrm{E}-0.1 \\
1,410 \mathrm{E}-01 \\
9,303 \mathrm{E}-0.2 \\
2.332 \mathrm{E}-02 \\
3.957 \mathrm{E}-03\end{array}$ & $\begin{array}{l}1.663 \mathrm{E}-01 \\
1.410 \mathrm{E}-01 \\
9.303 \mathrm{E}-02 \\
2.332 \mathrm{E}-02 \\
3.957 \mathrm{E}-0.3\end{array}$ \\
\hline $\begin{array}{l}5.000 \mathrm{E}-02 \\
6.000 \mathrm{E}-02 \\
7,000 \mathrm{E}-02 \\
8.000 \mathrm{E}-02 \\
9.000 \mathrm{E}-02\end{array}$ & $\begin{array}{l}5.608 \mathrm{E}-02 \\
5.826 \mathrm{E}-02 \\
6.043 \mathrm{E}-02 \\
6.258 \mathrm{E}-02 \\
6.473 \mathrm{E}-02\end{array}$ & $\begin{array}{l}5.608 \mathrm{E}-02 \\
5.826 \mathrm{E}-02 \\
6.043 \mathrm{E}-02 \\
6.258 \mathrm{E}-02 \\
6.473 \mathrm{E}-02\end{array}$ & $\begin{array}{l}5.000 \mathrm{E}+100 \\
6.000 \mathrm{E}+00 \\
7.000 \mathrm{E}+00 \\
8.000 \mathrm{E}+00 \\
9.000 \mathrm{E}+00\end{array}$ & $\begin{array}{l}1.233 \mathrm{E}-0.03 \\
1.008 \mathrm{E}-03 \\
9.971 \mathrm{E}-04 \\
9.968 \mathrm{E}-04 \\
9.968 \mathrm{E}-04\end{array}$ & $\begin{array}{l}1.233 \mathrm{E}-03 \\
1.008 \mathrm{E}-03 \\
9.971 \mathrm{E}-04 \\
9.968 \mathrm{E}-04 \\
9.968 \mathrm{E}-04\end{array}$ \\
\hline $\begin{array}{l}1,000 \mathrm{E}-01 \\
1.500 \mathrm{E}-01 \\
2.000 \mathrm{E}-0.01 \\
3.000 \mathrm{E}-01 \\
4.000 \mathrm{E}-01\end{array}$ & $\begin{array}{l}6.686 E-02 \\
7.727 E-02 \\
8.728 E-02 \\
1.059 E-01 \\
1.223 E-01\end{array}$ & $\begin{array}{l}6.686 \mathrm{E}-0.2 \\
7.727 \mathrm{E}-02 \\
8.728 \mathrm{E}-02 \\
1.059 \mathrm{E}-01 \\
1.223 \mathrm{E}-01\end{array}$ & $1.000 \mathrm{E}+01$ & $9.968 \mathrm{E}-04$ & $9,968 \mathrm{E}-04$ \\
\hline $\begin{array}{l}5.000 \mathrm{E}-01 \\
6.000 \mathrm{E}-01 \\
7.000 \mathrm{E}-01 \\
8.000 \mathrm{E}-01 \\
9.000 \mathrm{E}-01\end{array}$ & $\begin{array}{l}1.362 E-01 \\
1.476 E-01 \\
1.563 E-01 \\
1.622 E-01 \\
1.655 E-01\end{array}$ & $\begin{array}{l}1.362 E-01 \\
1.476 E-01 \\
1.563 E-01 \\
1.622 E-01 \\
1.655 E-01\end{array}$ & & & \\
\hline
\end{tabular}

* Based on Stefhest, 1970 
Table 2-21. Case 3 Results: Concentration of Np-237 in the Rock Matrix, at $x=100 \mathrm{~m}$, Time $t=10,000$ Years, Band Release Mode, Retardation Factor $\mathbf{R}^{\prime}=10,000$

\begin{tabular}{|c|c|c|c|c|c|}
\hline $\begin{array}{c}\text { Distance } \\
\mathbf{x}(\mathrm{m})\end{array}$ & FRACVAL & STEFH* & $\begin{array}{c}\text { Distance } \\
\mathrm{x}(\mathrm{m})\end{array}$ & RACVAL & STEFH ${ }^{*}$ \\
\hline $\begin{array}{l}1.000 \mathrm{E}-02 \\
1.500 \mathrm{E}-02 \\
2.000 \mathrm{E}-02 \\
3.000 \mathrm{E}-02 \\
4.000 \mathrm{E}-02\end{array}$ & $\begin{array}{l}1.073 E-01 \\
1.024 E-01 \\
9.750 E-02 \\
8.787 E-02 \\
7.859 E-02\end{array}$ & $\begin{array}{r}1.073 \mathrm{E}-01 \\
24 \mathrm{E}-01 \\
-750 \mathrm{E}-02 \\
8.787 \mathrm{E}-02 \\
7.859 \mathrm{E}-02\end{array}$ & $\begin{array}{l}1.000 \mathrm{E}-01 \\
1.500 \mathrm{E}-01 \\
2.000 \mathrm{E}-01 \\
3.000 \mathrm{E}-01 \\
4.000 \mathrm{E}-01\end{array}$ & $\begin{array}{l}3.485 \mathrm{E}-02 \\
1.521 \mathrm{E}-02 \\
6.181 \mathrm{E}-03 \\
1.468 \mathrm{E}-03 \\
1.023 \mathrm{E}-03\end{array}$ & $\begin{array}{l}3.48, \mathrm{~J}-02 \\
1.521 \mathrm{E}-02 \\
6.181 \mathrm{E}-03 \\
1.468 \mathrm{E}-03 \\
1.023 \mathrm{E}-03\end{array}$ \\
\hline $\begin{array}{l}5.000 \mathrm{E}-02 \\
6.000 \mathrm{E}-02 \\
7.000 \mathrm{E}-02 \\
8.000 \mathrm{E}-02 \\
9.000 \mathrm{E}-02\end{array}$ & $\begin{array}{l}6.978 E-02 \\
6.151 E-02 \\
5.387 E-02 \\
4.687 E-02 \\
4.053 E-02\end{array}$ & $\begin{array}{l}6.978 \mathrm{E}-02 \\
6.151 \mathrm{E}-02 \\
5.387 \mathrm{E}-02 \\
4.687 \mathrm{E}-02 \\
4.053 \mathrm{E}-02\end{array}$ & $\begin{array}{l}5.000 \mathrm{E}-01 \\
6.000 \mathrm{E}-01 \\
7.000 \mathrm{E}-01 \\
8.000 \mathrm{E}-01 \\
9.000 \mathrm{E}-01\end{array}$ & $\begin{array}{l}9.977 \mathrm{E}-04 \\
9.968 \mathrm{E}-04 \\
9.968 \mathrm{E}-04 \\
9.968 \mathrm{E}-04 \\
9.968 \mathrm{E}-04\end{array}$ & $\begin{array}{l}9.977 E-04 \\
9.968 E-04 \\
9.968 E-04 \\
9.968 E-04 \\
9.968 E-04\end{array}$ \\
\hline & & & $1.000 \mathrm{E}+00$ & $9.968 \mathrm{E}-04$ & $9.968 \mathrm{E}-04$ \\
\hline
\end{tabular}

* Based on Stefhest, 1970 


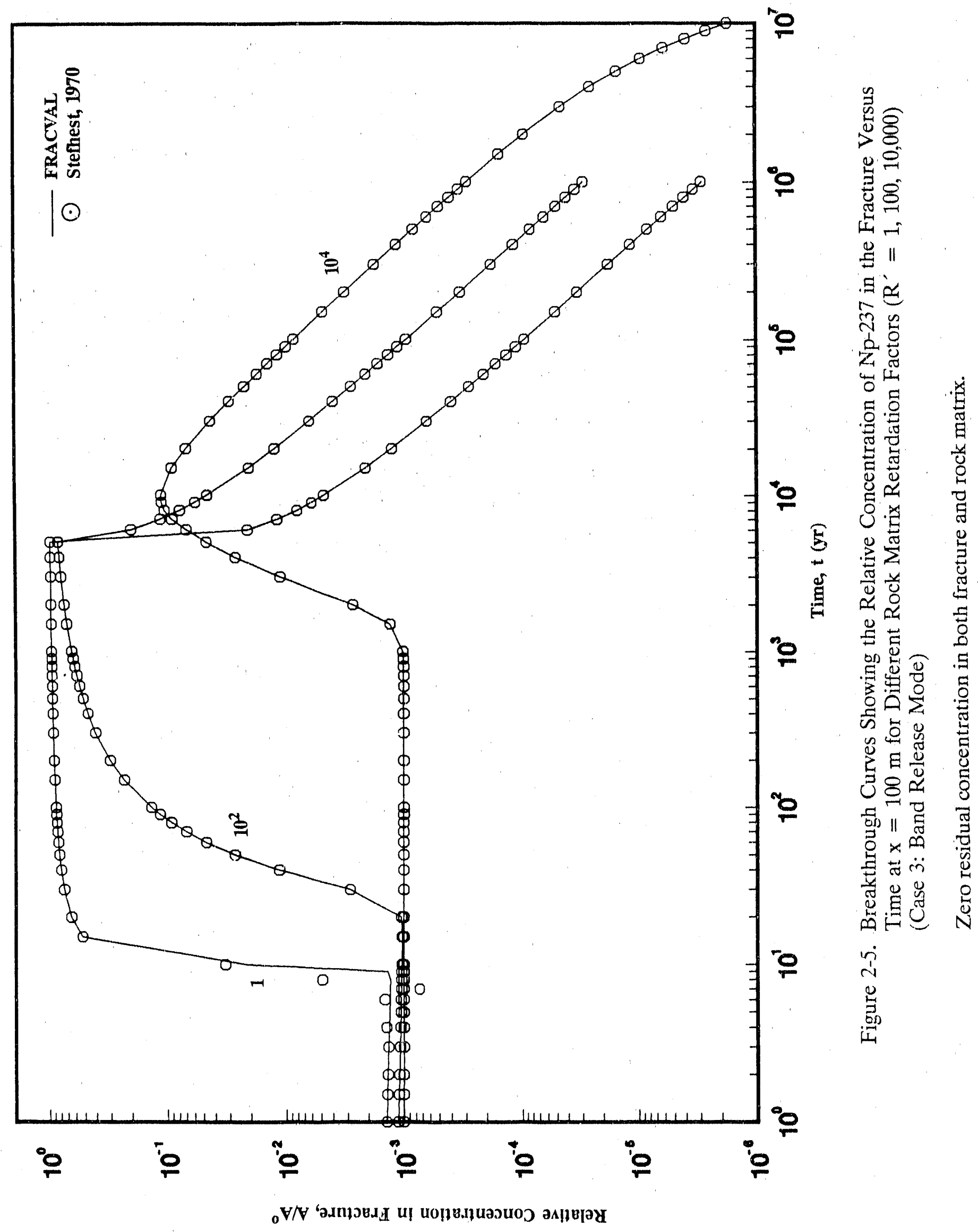




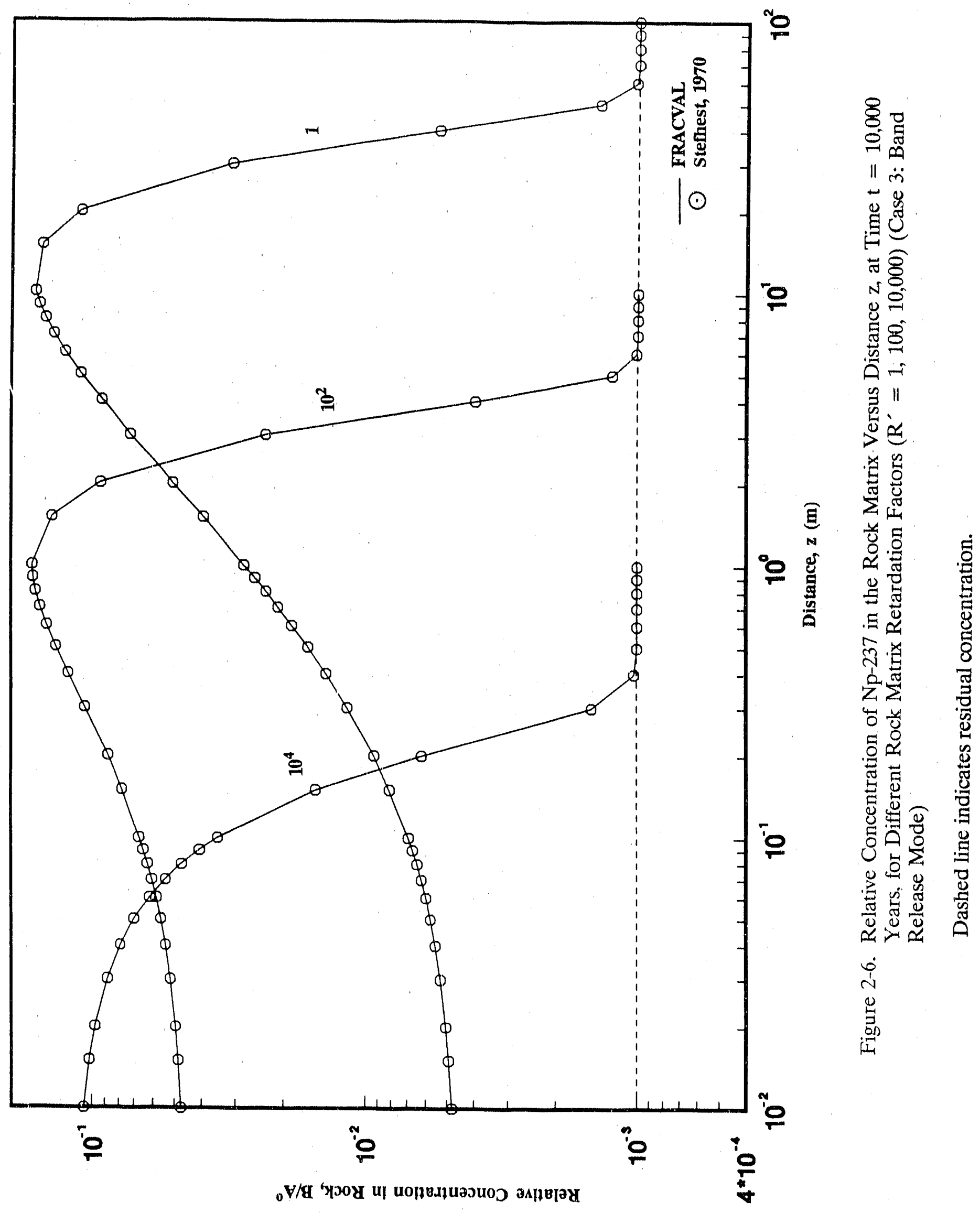


Table 2-22. Input Parameters for Case 4

\begin{tabular}{ll}
\hline \hline Species & Np-237 \\
Type of Release Mode & Band \\
Leaching Time T' & $10^{3} \mathrm{yr}$ \\
$\mathrm{T}_{1 / 2}$ & $2.14 \times 10^{6} \mathrm{yr}$ \\
$\mathrm{A}^{*}$ & 1.0 \\
$\mathrm{a}_{1}{ }^{*}$ & $1.5 \times 10^{-4}$ \\
$\mathrm{a}_{2}{ }^{*}$ & $-\left(0.5 \times 10^{-4}\right)^{* *}$ \\
$\mathrm{a}$ & $0.21 / \mathrm{m}$ \\
$\mathrm{b}_{1}{ }^{*}$ & $1.0 \times 10^{-5}$ \\
$\mathrm{D}_{\mathrm{p}}$ & $0.01 \mathrm{~m} / \mathrm{yr}$ \\
$\mathrm{u}$ & $10.0 \mathrm{~m} / \mathrm{yr}$ \\
$\mathrm{D}_{\mathrm{x}}$ & $0,10,100$, \\
& $1,000,10,000$ \\
$\mathrm{~b}$ & $\mathrm{~m} 2 / \mathrm{yr}$ \\
$\mathrm{R}$ & $0.005 \mathrm{~m}$ \\
$\mathrm{R}^{\prime}$ & 3.0 \\
$\mathrm{t}$ & 1,000 \\
\hline & $5,000 \mathrm{yr}$ \\
\hline
\end{tabular}

*(arbitrary units of activity/Li?)

**'This indicates that in Equation 2-7 the constant

$+\mathrm{a}_{2}$ becomes - $\mathrm{a}_{2}$.

at the source, the concentration gradient is such that transfer of $\mathrm{Np}-237$ must proceed from fracture to rock matrix until the concentration equalizes on each side of the fracture rock interface. 
Table 2-23. Case 4 Results: Concentration of Np-237 in the Fracture, at Time $t=5,0(0)$ Years, Band Release Mode, Zero Dispersion, $D_{x}=0 \mathrm{~m}^{2} / \mathrm{yr}$

\begin{tabular}{ccc}
\hline & & \\
\hline & & \\
Distance & & \\
$\mathbf{x}(\mathrm{m})$ & FRACVAL & STEFH* \\
\hline & & \\
$1.000 \mathrm{E}+00$ & $5.948 \mathrm{E}-04$ & $5.948 \mathrm{E}-04$ \\
$1.500 \mathrm{E}+00$ & $8.922 \mathrm{E}-04$ & $8.922 \mathrm{E}-04$ \\
$2.000 \mathrm{E}+00$ & $1.190 \mathrm{E}-03$ & $1.190 \mathrm{E}-03$ \\
$3.000 \mathrm{E}+00$ & $1.784 \mathrm{E}-03$ & $1.784 \mathrm{E}-03$ \\
$4.000 \mathrm{E}+00$ & $2.379 \mathrm{E}-03$ & $2.379 \mathrm{E}-03$ \\
& & \\
$5.000 \mathrm{E}+00$ & $2.973 \mathrm{E}-03$ & $2.973 \mathrm{E}-03$ \\
$6.000 \mathrm{E}+00$ & $3.568 \mathrm{E}-03$ & $3.568 \mathrm{E}-03$ \\
$7.000 \mathrm{E}+00$ & $4.161 \mathrm{E}-03$ & $4.161 \mathrm{E}-03$ \\
$8.000 \mathrm{E}+00$ & $4.755 \mathrm{E}-03$ & $4.755 \mathrm{E}-03$ \\
$9.000 \mathrm{E}+00$ & $5.348 \mathrm{E}-03$ & $5.348 \mathrm{E}-03$ \\
& & \\
$1.000 \mathrm{E}+01$ & $5.940 \mathrm{E}-03$ & $5.940 \mathrm{E}-03$ \\
$1.500 \mathrm{E}+01$ & $8.889 \mathrm{E}-03$ & $8.889 \mathrm{E}-03$ \\
$2.000 \mathrm{E}+01$ & $1.181 \mathrm{E}-02$ & $1.181 \mathrm{E}-02$ \\
$3.000 \mathrm{E}+01$ & $1.754 \mathrm{E}-02$ & $1.754 \mathrm{E}-02$ \\
$4.000 \mathrm{E}+01$ & $2.304 \mathrm{E}-02$ & $2.304 \mathrm{E}-02$ \\
& & \\
$5.000 \mathrm{E}+01$ & $2.825 \mathrm{E}-02$ & $2.825 \mathrm{E}-02$ \\
$6.000 \mathrm{E}+01$ & $3.310 \mathrm{E}-02$ & $3.310 \mathrm{E}-02$ \\
$7.000 \mathrm{E}+01$ & $3.754 \mathrm{E}-02$ & $3.754 \mathrm{E}-02$ \\
$8.000 \mathrm{E}+01$ & $4.151 \mathrm{E}-02$ & $4.151 \mathrm{E}-02$ \\
$9.000 \mathrm{E}+01$ & $4.499 \mathrm{E}-02$ & $4.499 \mathrm{E}-02$ \\
& & \\
$1.000 \mathrm{E}+02$ & $4.793 \mathrm{E}-02$ & $4.793 \mathrm{E}-02$ \\
$1.500 \mathrm{E}+02$ & $5.440 \mathrm{E}-02$ & $5.440 \mathrm{E}-02$ \\
$2.000 \mathrm{E}+02$ & $4.893 \mathrm{E}-02$ & $4.893 \mathrm{E}-02$ \\
$3.000 \mathrm{E}+02$ & $2.360 \mathrm{E}-02$ & $2.360 \mathrm{E}-02$ \\
$4.000 \mathrm{E}+02$ & $6.364 \mathrm{E}-03$ & $6.364 \mathrm{E}-03$ \\
& & \\
$5.000 \mathrm{E}+02$ & $1.019 \mathrm{E}-03$ & $1.019 \mathrm{E}-03$ \\
$6.000 \mathrm{E}+02$ & $1.073 \mathrm{E}-04$ & $1.073 \mathrm{E}-04$ \\
$7.000 \mathrm{E}+02$ & $1.623 \mathrm{E}-05$ & $1.623 \mathrm{E}-05$ \\
$8.000 \mathrm{E}+02$ & $1.072 \mathrm{E}-05$ & $1.072 \mathrm{E}-05$ \\
$9.000 \mathrm{E}+02$ & $1.052 \mathrm{E}-05$ & $1.052 \mathrm{E}-05$ \\
$1.000 \mathrm{E}+03$ & $1.051 \mathrm{E}-05$ & $1.051 \mathrm{E}-05$ \\
$1.500 \mathrm{E}+03$ & $1.051 \mathrm{E}-05$ & $1.051 \mathrm{E}-05$ \\
$2.000 \mathrm{E}+03$ & $1.051 \mathrm{E}-05$ & $1.051 \mathrm{E}-05$ \\
\hline
\end{tabular}

* Based on Stefhest, 1970 
Table 2-24. Case 4 Results: Concentration of Np-237 in the Fracture, at Time $t=5,000$ Years, Band Release Mode, $D_{x}=10 \mathrm{~m}^{2} / \mathrm{yr}$

\begin{tabular}{lll}
\hline & & \\
Distance & & \\
$\mathbf{x}(\mathrm{m})$ & FRACVAL & STEFH \\
\hline & & \\
\hline & & \\
$1.000 \mathrm{E}+00$ & $5.946 \mathrm{E}-04$ & $5.946 \mathrm{E}-04$ \\
$1.500 \mathrm{E}+00$ & $8.919 \mathrm{E}-04$ & $8.919 \mathrm{E}-04$ \\
$2.000 \mathrm{E}+00$ & $1.189 \mathrm{E}-03$ & $1.189 \mathrm{E}-03$ \\
$3.000 \mathrm{E}+00$ & $1.783 \mathrm{E}-03$ & $1.783 \mathrm{E}-03$ \\
$4.000 \mathrm{E}+00$ & $2.378 \mathrm{E}-03$ & $2.378 \mathrm{E}-03$ \\
& & \\
$5.000 \mathrm{E}+00$ & $2.971 \mathrm{E}-03$ & $2.971 \mathrm{E}-03$ \\
$6.000 \mathrm{E}+00$ & $3.564 \mathrm{E}-03$ & $3.564 \mathrm{E}-03$ \\
$7.000 \mathrm{E}+00$ & $4.157 \mathrm{E}-03$ & $4.157 \mathrm{E}-03$ \\
$8.000 \mathrm{E}+00$ & $4.749 \mathrm{E}-03$ & $4.749 \mathrm{E}-03$ \\
$9.000 \mathrm{E}+00$ & $5.341 \mathrm{E}-03$ & $5.341 \mathrm{E}-03$ \\
& & \\
$1.000 \mathrm{E}+01$ & $5.931 \mathrm{E}-03$ & $5.931 \mathrm{E}-03$ \\
$1.500 \mathrm{E}+01$ & $8.871 \mathrm{E}-03$ & $8.871 \mathrm{E}-03$ \\
$2.000 \mathrm{E}+01$ & $1.178 \mathrm{E}-02$ & $1.178 \mathrm{E}-02$ \\
$3.000 \mathrm{E}+01$ & $1.747 \mathrm{E}-02$ & $1.747 \mathrm{E}-02$ \\
$4.000 \mathrm{E}+01$ & $2.292 \mathrm{E}-02$ & $2.292 \mathrm{E}-02$ \\
& & \\
$5.000 \mathrm{E}+01$ & $2.807 \mathrm{E}-02$ & $2.807 \mathrm{E}-02$ \\
$6.000 \mathrm{E}+01$ & $3.285 \mathrm{E}-02$ & $3.285 \mathrm{E}-02$ \\
$7.000 \mathrm{E}+01$ & $3.721 \mathrm{E}-02$ & $3.721 \mathrm{E}-02$ \\
$8.000 \mathrm{E}+01$ & $4.111 \mathrm{E}-02$ & $4.111 \mathrm{E}-02$ \\
$9.000 \mathrm{E}+01$ & $4.451 \mathrm{E}-02$ & $4.451 \mathrm{E}-02$ \\
& & \\
$1.000 \mathrm{E}+02$ & $4.738 \mathrm{E}-02$ & $4.738 \mathrm{E}-02$ \\
$1.500 \mathrm{E}+02$ & $5.368 \mathrm{E}-02$ & $5.368 \mathrm{E}-02$ \\
$2.000 \mathrm{E}+02$ & $4.841 \mathrm{E}-02$ & $4.841 \mathrm{E}-02$ \\
$3.000 \mathrm{E}+02$ & $2.394 \mathrm{E}-02$ & $2.394 \mathrm{E}-02$ \\
$4.000 \mathrm{E}+02$ & $6.854 \mathrm{E}-03$ & $6.854 \mathrm{E}-03$ \\
& & \\
$5.000 \mathrm{E}+02$ & $1.217 \mathrm{E}-03$ & $1.217 \mathrm{E}-03$ \\
$6.000 \mathrm{E}+02$ & $1.463 \mathrm{E}-04$ & $1.463 \mathrm{E}-04$ \\
$7.000 \mathrm{E}+02$ & $2.051 \mathrm{E}-05$ & $2.051 \mathrm{E}-05$ \\
$8.000 \mathrm{E}+02$ & $1.100 \mathrm{E}-05$ & $1.100 \mathrm{E}-05$ \\
$9.000 \mathrm{E}+02$ & $1.053 \mathrm{E}-05$ & $1.053 \mathrm{E}-05$ \\
$1.000 \mathrm{E}+03$ & $1.051 \mathrm{E}-05$ & $1.051 \mathrm{E}-05$ \\
$1.500 \mathrm{E}+03$ & $1.051 \mathrm{E}-05$ & $1.051 \mathrm{E}-05$ \\
$2.000 \mathrm{E}+03$ & $1.051 \mathrm{E}-05$ & $1.051 \mathrm{E}-05$ \\
$3.000 \mathrm{E}+03$ & $1.051 \mathrm{E}-05$ & $1.051 \mathrm{E}-05$ \\
\hline \hline
\end{tabular}

* Based on Stefhest, 1970 
Table 2-25. Case 4 Results: Concentration of Np-237 in the Fracture, at Time $\mathrm{t}=5,000$ Years, Band Release Mode, $\mathrm{D}_{\mathrm{x}}=100 \mathrm{~m}^{2} / \mathrm{yr}$

\begin{tabular}{ccc}
\hline & & \\
\hline & & \\
Distance & & \\
$\mathbf{x}(\mathrm{m})$ & FRACVAL & STEFH \\
\hline & & \\
$1.000 \mathrm{E}+00$ & $5.810 \mathrm{E}-04$ & $5.810 \mathrm{E}-04$ \\
$1.500 \mathrm{E}+00$ & $8.710 \mathrm{E}-04$ & $8.710 \mathrm{E}-04$ \\
$2.000 \mathrm{E}+00$ & $1.161 \mathrm{E}-03$ & $1.161 \mathrm{E}-03$ \\
$3.000 \mathrm{E}+00$ & $1.739 \mathrm{E}-03$ & $1.739 \mathrm{E}-03$ \\
$4.000 \mathrm{E}+00$ & $2.316 \mathrm{E}-03$ & $2.316 \mathrm{E}-03$ \\
& & \\
$5.000 \mathrm{E}+00$ & $2.891 \mathrm{E}-03$ & $2.891 \mathrm{E}-03$ \\
$6.000 \mathrm{E}+00$ & $3.464 \mathrm{E}-03$ & $3.464 \mathrm{E}-03$ \\
$7.000 \mathrm{E}+00$ & $4.036 \mathrm{E}-03$ & $4.036 \mathrm{E}-03$ \\
$8.000 \mathrm{E}+00$ & $4.606 \mathrm{E}-03$ & $4.606 \mathrm{E}-03$ \\
$9.000 \mathrm{E}+00$ & $5.174 \mathrm{E}-03$ & $5.174 \mathrm{E}-03$ \\
& & \\
$1.000 \mathrm{E}+01$ & $5.741 \mathrm{E}-03$ & $5.741 \mathrm{E}-03$ \\
$1.500 \mathrm{E}+01$ & $8.541 \mathrm{E}-03$ & $8.541 \mathrm{E}-03$ \\
$2.000 \mathrm{E}+01$ & $1.129 \mathrm{E}-02$ & $1.129 \mathrm{E}-02$ \\
$3.000 \mathrm{E}+01$ & $1.657 \mathrm{E}-02$ & $1.657 \mathrm{E}-02$ \\
$4.000 \mathrm{E}+01$ & $2.156 \mathrm{E}-02$ & $2.156 \mathrm{E}-02$ \\
& & \\
$5.000 \mathrm{E}+01$ & $2.618 \mathrm{E}-02$ & $2.618 \mathrm{E}-02$ \\
$6.000 \mathrm{E}+01$ & $3.042 \mathrm{E}-02$ & $3.042 \mathrm{E}-02$ \\
$7.000 \mathrm{E}+01$ & $3.423 \mathrm{E}-02$ & $3.423 \mathrm{E}-02$ \\
$8.000 \mathrm{E}+01$ & $3.760 \mathrm{E}-02$ & $3.760 \mathrm{E}-02$ \\
$9.000 \mathrm{E}+01$ & $4.051 \mathrm{E}-02$ & $4.051 \mathrm{E}-02$ \\
$1.000 \mathrm{E}+02$ & $4.296 \mathrm{E}-02$ & $4.296 \mathrm{E}-02$ \\
$1.500 \mathrm{E}+02$ & $4.855 \mathrm{E}-02$ & $4.855 \mathrm{E}-02$ \\
$2.000 \mathrm{E}+02$ & $4.504 \mathrm{E}-02$ & $4.504 \mathrm{E}-02$ \\
$3.000 \mathrm{E}+02$ & $2.627 \mathrm{E}-02$ & $2.627 \mathrm{E}-02$ \\
$4.000 \mathrm{E}+02$ & $1.045 \mathrm{E}-02$ & $1.045 \mathrm{E}-02$ \\
& & \\
$5.000 \mathrm{E}+02$ & $3.068 \mathrm{E}-03$ & $3.068 \mathrm{E}-03$ \\
$6.000 \mathrm{E}+02$ & $7.001 \mathrm{E}-04$ & $7.001 \mathrm{E}-04$ \\
$7.000 \mathrm{E}+02$ & $1.339 \mathrm{E}-04$ & $1.339 \mathrm{E}-04$ \\
$8.000 \mathrm{E}+02$ & $2.840 \mathrm{E}-05$ & $2.840 \mathrm{E}-05$ \\
$9.000 \mathrm{E}+02$ & $1.265 \mathrm{E}-05$ & $1.265 \mathrm{E}-05$ \\
& & \\
$1.000 \mathrm{E}+03$ & $1.073 \mathrm{E}-05$ & $1.073 \mathrm{E}-05$ \\
$1.500 \mathrm{E}+03$ & $1.051 \mathrm{E}-05$ & $1.051 \mathrm{E}-05$ \\
$2.000 \mathrm{E}+03$ & $1.051 \mathrm{E}-05$ & $1.051 \mathrm{E}-05$ \\
$3.000 \mathrm{E}+03$ & $1.051 \mathrm{E}-05$ & $1.051 \mathrm{E}-05$ \\
$4.000 \mathrm{E}+03$ & $1.051 \mathrm{E}-05$ & $1.051 \mathrm{E}-05$ \\
\hline
\end{tabular}

* Based on Stefhest, 1970 
Table 2.26. Case 4 Results: Concentration of Np-237 in the Fracture, ut Time $1=5,000$ Years, Bund Release Mode, $D_{x}=1,000 \mathrm{~m}^{2} / \mathrm{yr}$

\begin{tabular}{|c|c|c|c|c|c|}
\hline $\begin{array}{l}\text { Distance } \\
\mathrm{x}(\mathrm{m})\end{array}$ & FRACVAL & STEFH* & $\begin{array}{l}\text { Distance } \\
\mathrm{x}(\mathrm{m})\end{array}$ & FRACVAL & STEFH* \\
\hline $1.000 \mathrm{E}+00$ & $3.764 \mathrm{E}-04$ & $3.765 \mathrm{E}-04$ & $1,000 \mathrm{E}+02$ & $2.684 E-112$ & $2.684 \mathrm{E}-(12$ \\
\hline $1.500 \mathrm{E}+00$ & $5.639 \mathrm{E}-04$ & $5.639 \mathrm{E}-04$ & $1.500 \mathrm{E}+02$ & $3.273 \mathrm{E}-02$ & $3.273 E-02$ \\
\hline $2.000 \mathrm{E}+00$ & $7.508 \mathrm{E}-04$ & $7.508 \mathrm{E}-04$ & $2.0 \mathrm{OOOE}+02$ & $3.473 E-02$ & $3.473 \mathrm{E}-(12$ \\
\hline $3.000 \mathrm{E}+00$ & $1.123 \mathrm{E}-03$ & $1.123 \mathrm{E}-03$ & $3.000 \mathrm{E}+02$ & $3.111 \mathrm{E}-02$ & $3.111 \mathrm{E}-0)^{2}$ \\
\hline $4.000 E+00$ & $1.493 E-03$ & $1.493 \mathrm{E}-03$ & $4.000 \mathrm{E}+02$ & $2.310 \mathrm{E}-02$ & $2.310 \mathrm{E}-02$ \\
\hline $5.000 \mathrm{E}+00$ & $1.861 \mathrm{E}-03$ & $1.861 E-03$ & $5.000 \mathrm{E}+02$ & $1.511 \mathrm{E}-02$ & $1.511 E-(02$ \\
\hline $6.000 \mathrm{E}+00$ & $2.226 \mathrm{E}-03$ & $2.226 \mathrm{E}-03$ & $6.0000 \mathrm{E}+02$ & $8.973 E \cdots() 3$ & $8.973 E-03$ \\
\hline $7.000 \mathrm{E}+00$ & $2.589 E-03$ & $2.589 \mathrm{E}-03$ & $7.000 \mathrm{E}+02$ & $4.920 \mathrm{E}-03$ & $4.920 E-(13$ \\
\hline $8.000 \mathrm{E}+00$ & $2.951 \mathrm{E}-03$ & $2.951 \mathrm{E}-03$ & $8.000 \mathrm{E}+02$ & $2.522 \mathrm{E}-03$ & $2.522 \mathrm{E}-03$ \\
\hline $9.000 \mathrm{E}+00$ & $3.310 \mathrm{E}-03$ & $3.310 \mathrm{E}-03$ & $9.000 \mathrm{E}+02$ & $1.220 \mathrm{E}-03$ & $1.220 \mathrm{E}-03$ \\
\hline $1.000 \mathrm{E}+01$ & $3.666 \mathrm{E}-03$ & $3.666 \mathrm{E}-03$ & $1.000 \mathrm{E}+03$ & $5.620 \mathrm{E}-04$ & $5.620 \mathrm{E}-04$ \\
\hline $1.500 \mathrm{E}+01$ & $5.417 E-03$ & $5.417 \mathrm{E}-03$ & $1.500 \mathrm{E}+03$ & $1.604 E-05$ & $1.604 E-05$ \\
\hline $2.000 \mathrm{E}+01$ & $7.113 E-03$ & $7.113 E-03$ & $2.000 E+03$ & $1.054 \mathrm{E}-0.5$ & $1.054 E-05$ \\
\hline $3.000 \mathrm{E}+01$ & $1.034 \mathrm{E}-02$ & $1.034 \mathrm{E}-02$ & $3.000 \mathrm{E}+03$ & $1.051 \mathrm{E}-0.5$ & $1.051 \mathrm{E}-0.5$ \\
\hline $4.000 \mathrm{E}+01$ & $1.334 E-02$ & $1.334 \mathrm{E}-02$ & $4.000 \mathrm{E}+03$ & $1.051 \mathrm{E}-05$ & $1.051 \mathrm{E}-05$ \\
\hline $5.000 \mathrm{E}+01$ & $1.612 \mathrm{E}-02$ & $1.612 \mathrm{E}-02$ & $5.000 \mathrm{E}+03$ & $1.051 E-0.5$ & $1.051 E-05$ \\
\hline $6.000 \mathrm{E}+01$ & $1.869 E-02$ & $1.869 \mathrm{E}-02$ & $6.000 E+03$ & $1.051 \mathrm{E}-0.0$ & $1.051 E-05$ \\
\hline $7.000 \mathrm{E}+01$ & $2.104 E-02$ & $2.104 \mathrm{E}-02$ & $7.000 \mathrm{E}+03$ & $1.051 \mathrm{E}-05$ & $1.051 \mathrm{E}-05$ \\
\hline $8.000 \mathrm{E}+0.1$ & $2.318 \mathrm{E}-02$ & $2.318 \mathrm{E}-02$ & $8.000 \mathrm{E}+03$ & $1.051 E-0.5$ & $1.051 \mathrm{E}-0.5$ \\
\hline \multirow[t]{2}{*}{$9.000 \mathrm{E}+01$} & $2.511 E-02$ & $2.511 \mathrm{E}-02$ & $9.000 \mathrm{E}+03$ & $1.051 \mathrm{E}-05$ & $1.051 E-05$ \\
\hline & & & $1.000 \mathrm{E}+04$ & $1.051 \mathrm{E}-0.5$ & $1.051 E-05$ \\
\hline
\end{tabular}

* Based on Stefhest, 1970 
Table 2-27. Case 4 Results: Concentration of Np-237 in the Fracture, at Time $t=5,000$ Years, Band Releaso Mode, $D_{x}=10,000 \mathrm{~m}^{2} / \mathrm{yr}$

\begin{tabular}{|c|c|c|c|c|c|}
\hline $\begin{array}{c}\text { Distance } \\
\mathrm{x}(\mathrm{m})\end{array}$ & FRACVAL & STEFH* & $\begin{array}{l}\text { Distance } \\
\mathbf{x}(\mathrm{m})\end{array}$ & FRACVAL & STEFH* \\
\hline $1.000 \mathrm{E}+00$ & $1.372 E-04$ & $1.376 \mathrm{E}-04$ & $1.000 E+02$ & $1.161 \mathrm{E}-02$ & $1.161 \mathrm{E}-02$ \\
\hline $1.500 \mathrm{E}+00$ & $2.058 \mathrm{E}-04$ & $2.063 \mathrm{E}-04$ & $1.500 \mathrm{E}+02$ & $1.590 \mathrm{E}-02$ & $1.590 \mathrm{E}-02$ \\
\hline $2.000 \mathrm{E}+00$ & $2.743 E-04$ & $2.748 \mathrm{E}-04$ & $2.000 E+02$ & $1.930 \mathrm{E}-02$ & $1,930 \mathrm{E}-02$ \\
\hline $3,000 \mathrm{E}+00$ & $4.111 E-04$ & $4.115 E-04$ & $3,000 \mathrm{E}+02$ & $2.381 \mathrm{E}-02$ & $2.381 E-02$ \\
\hline $4.000 \mathrm{E}+00$ & $5.474 E-04$ & $5.478 \mathrm{E}-04$ & $4.000 \mathrm{E}+02$ & $2.583 E-02$ & $2.583 \mathrm{E}-02$ \\
\hline $5.000 \mathrm{E}+00$ & $6.832 \mathrm{E}-04$ & $6.836 \mathrm{E}-04$ & $5.000 \mathrm{E}+02$ & $2.601 E-02$ & $2.601 \mathrm{E}-02$ \\
\hline $6.000 \mathrm{E}+00$ & $8.186 \mathrm{E}-04$ & $8.190 E-04$ & $6.000 \mathrm{E}+02$ & $2.491 E-02$ & $2.491 \mathrm{E}-02$ \\
\hline $7.000 \mathrm{E}+00$ & $9.536 \mathrm{E}-04$ & $9.539 E-04$ & $7.000 \mathrm{E}+02$ & $2.299 \mathrm{E}-02$ & $2.299 \mathrm{E}-02$ \\
\hline $8.000 \mathrm{E}+00$ & $1.088 \mathrm{E}-03$ & $1.088 E-03$ & $8.000 \mathrm{E}+02$ & $2.061 E-02$ & $2.061 \mathrm{E}-02$ \\
\hline $9.000 \mathrm{E}+00$ & $1.222 \mathrm{E}-03$ & $1.222 \mathrm{E}-03$ & $9.000 \mathrm{E}+02$ & $1.804 \mathrm{E}-02$ & $1.804 E-02$ \\
\hline $1.000 \mathrm{E}+01$ & $1.356 \mathrm{E}-03$ & $1.356 \mathrm{E}-03$ & $1.000 E+03$ & $1.548 \mathrm{E}-02$ & $1.548 \mathrm{E}-02$ \\
\hline $1.500 \mathrm{E}+01$ & $2.017 \mathrm{E}-03$ & $2.017 \mathrm{E}-03$ & $1.500 E+03$ & $5.742 E-03$ & $5.742 \mathrm{E}-03$ \\
\hline $2.000 \mathrm{E}+01$ & $2.667 \mathrm{E}-03$ & $2.667 E-03$ & $2.000 E+03$ & $1.628 E-03$ & $1.628 \mathrm{E}-03$ \\
\hline $3.000 E+01$ & $3.933 E-03$ & $3.933 E-03$ & $3.000 \mathrm{E}+03$ & $8.447 \mathrm{E}-05$ & $8.447 \mathrm{E}-0.5$ \\
\hline $4.000 \mathrm{E}+01$ & $5.155 E-03$ & $5.155 E-03$ & $4.000 E+03$ & $1.247 \mathrm{E}-0.5$ & $1.247 \mathrm{E}-05$ \\
\hline $5,000 E+01$ & $6.335 E-03$ & $6.335 E-03$ & $5.000 \mathrm{E}+03$ & $1.055 E-05$ & $1.055 \mathrm{E}-05$ \\
\hline $6.0010 \mathrm{E}+01$ & $7.471 E-03$ & $7.471 E-03$ & $6.000 \mathrm{E}+03$ & $1.051 E-05$ & $1.051 \mathrm{E}-05$ \\
\hline $7,000 \mathrm{E}+01$ & $8.566 E-03$ & $8.566 E-03$ & $7.000 \mathrm{E}+03$ & $1.051 \mathrm{E}-05$ & $1.051 \mathrm{E}-0.5$ \\
\hline $8.00013+01$ & $9.620 \mathrm{E}-03$ & $9.620 E-03$ & $8.000 \mathrm{E}+03$ & $1.051 E-05$ & $1.051 \mathrm{E}-0.5$ \\
\hline $9.000 \mathrm{E}+01$ & $1.063 \mathrm{E}-02$ & $1.063 E-02$ & $9.000 \mathrm{E}+03$ & $1.051 \mathrm{E}-0.5$ & $1.051 E-0.5$ \\
\hline & & & $1.000 \mathrm{E}+04$ & $1.051 E-05$ & $1.051 \mathrm{E}-05$ \\
\hline
\end{tabular}

* Based on Stefhest, 1970 
Tuble 2.28. Case 4 Results: Concentration of Npu237 ln the Rock Mutrix, at $x=100 \mathrm{~m}$, 'I'me $1=5,0(0)$ Years, Band Release Mode, Zero Disperston, D $0 \mathrm{~m}^{2} / \mathrm{yr}^{\prime}$

\begin{tabular}{|c|c|c|c|c|c|}
\hline $\begin{array}{c}\text { Distance } \\
\times(\mathrm{m})\end{array}$ & FRACVAL & S'TEFH* & $\begin{array}{l}\text { Distance } \\
x(\mathrm{~m})\end{array}$ & FRACVAL. & S'TELH" \\
\hline $1.0000 \mathrm{E}-02$ & $4.857 \mathrm{E}-02$ & $4.857 \mathrm{E}-02$ & $1.000 \mathrm{E}+00$ & $1.380 \mathrm{E}-() 4$ & $1,3801 \mathrm{E}-0.4$ \\
\hline $1.500 \mathrm{E}-02$ & $4.917 \mathrm{E}-02$ & $4.917 \mathrm{E}-02$ & $1.500 \mathrm{E}+00$ & $1.006 \mathrm{E}-0.5$ & $1,006 \mathrm{E}-0.5$ \\
\hline $2.000 E-02$ & $4.974 \mathrm{E}-02$ & $4,974 \mathrm{E}-02$ & $2 .(00) \mathrm{E}+(0)$ & $9,984 \mathrm{E}-06$ & $9.984 \mathrm{E}-1) 6$ \\
\hline $3.000 \mathrm{E}-02$ & $5.078 \mathrm{E}-02$ & $5 .(078 \mathrm{E}-02$ & $3,000 \mathrm{E}+00$ & $0.984 \mathrm{E}-06$ & $9.984 E-06$ \\
\hline $4.000 \mathrm{E}-02$ & $5.168 \mathrm{E}-02$ & $5.168 \mathrm{E}-02$ & $4.000 \mathrm{E}+00$ & $9.984 E-06$ & $9.984 \mathrm{E}-06$ \\
\hline $5.000 \mathrm{E}-02$ & $5.244 \mathrm{E}-02$ & $5,244 E-02$ & $5.000 \mathrm{E}+00)$ & $9,984 \mathrm{E} \cdots 06$ & $9.984 \mathrm{E}-\mathrm{-06}$ \\
\hline $6.000 \mathrm{E}-02$ & $5.306 \mathrm{E}-() 2$ & $5,3() 6 \mathrm{E}-() 2$ & $6,000 \mathrm{E}+00$ & $9,984 \mathrm{E}-06$ & $9.984 \mathrm{E}-\ldots, 06$ \\
\hline $7.000 \mathrm{E}-02$ & $5.354 \mathrm{E}-02$ & $5,354 E-02$ & $7,000 \mathrm{E}+(0)$ & $9.984 \mathrm{E}-06$ & $9.984 \mathrm{E}-\ldots 06$ \\
\hline $8.000 \mathrm{E}-02$ & $5.390 \mathrm{E}-02$ & $5.390 \mathrm{E}-02$ & $8,000 \mathrm{E}+(0)$ & $9.984 E-06$ & $9.984 E-06$ \\
\hline $9,000 \mathrm{E}-02$ & $5.412 \mathrm{E}-02$ & $5.412 \mathrm{E}-02$ & $9,000 \mathrm{E}+00$ & $9.984 E-06$ & $9,984 \mathrm{E}-06$ \\
\hline $1.000 \mathrm{E}-01$ & $5.42 .2 \mathrm{E}-02$ & $5.422 E-(02$ & $1.000 \mathrm{E}+01$ & $9.984 E-06$ & $9.984 \mathrm{E}-06$ \\
\hline $1.500 \mathrm{E}-01$ & $5.294 \mathrm{E}-\mathrm{0}$ & $5.294 \mathrm{E}-02$ & & & \\
\hline $2.000 \mathrm{E}-01$ & $4.921 E-02$ & $4,921 \mathrm{E}-02$ & & & \\
\hline $3.000 E-01$ & $3.738 E-02$ & $3.738 \mathrm{E}-02$ & & & \\
\hline $4.000 \mathrm{E}-01$ & $2.438 E-02$ & $2.438 \mathrm{E}-02$ & & & \\
\hline $5.000 \mathrm{E}-01$ & $1.384 E-02$ & $1.384 \mathrm{E}-02$ & & & \\
\hline $6.000 \mathrm{E}-01$ & $6.899 \mathrm{E}-03$ & $6.899 \mathrm{E}-03$ & & & \\
\hline $7,000 E-01$ & $3.042 E-03$ & $3.042 E-03$ & & & \\
\hline $8.000 E-01$ & $1.194 \mathrm{E}-03$ & $1.194 E-03$ & & & \\
\hline $9.000 \mathrm{E}-01$ & $4.218 \mathrm{E}-04$ & $4.218 \mathrm{E}-04$ & & & \\
\hline
\end{tabular}

* Based on Stefhest, 1970) 
Tablo 2.29, Caso 4 Reosults: Concontration of Np-237 in the Rock Matrix, it

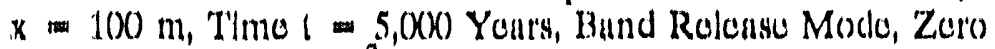
Disporslon, $D=10 \mathrm{~m}^{2} / \mathrm{yr}^{\mathrm{r}}$

\begin{tabular}{|c|c|c|}
\hline $\begin{array}{c}\text { Distance } \\
x(n 1)\end{array}$ & FRACVAL & STEFH* \\
\hline $\begin{array}{l}1.000 E-02 \\
1.500 E-02 \\
2.000 E-102 \\
3.000 E-012 \\
4.000(0 E-02\end{array}$ & $\begin{array}{l}4,802 \mathrm{E}-02 \\
4,86^{\prime} \mathrm{E}-02 \\
4,919 \mathrm{E}-02 \\
5,023 \mathrm{E}-02 \\
5.113 \mathrm{E}-02\end{array}$ & $\begin{array}{l}4.802 E-02 \\
4.862+-02 \\
4.91, E-02 \\
5.023 E-02 \\
5.113 E-02\end{array}$ \\
\hline $\begin{array}{l}5.000 \mathrm{E}-02 \\
6.000 \mathrm{E}-02 \\
7.000 \mathrm{E}-02 \\
8.000 \mathrm{E}-02 \\
9.000 \mathrm{E}-02\end{array}$ & $\begin{array}{l}5.189 \mathrm{E}-02 \\
5.252 \mathrm{E}-02 \\
5.302 \mathrm{E}-02 \\
5.338 \mathrm{E}-02 \\
5.362 \mathrm{E}-02\end{array}$ & $\begin{array}{l}5.189 E-02 \\
5.252 E-02 \\
5.302 E-02 \\
5.338 E-02 \\
5.362 E-02\end{array}$ \\
\hline $\begin{array}{l}1.000 \mathrm{E}-01 \\
1.500 \mathrm{E}-01 \\
2.000 \mathrm{E}-01 \\
3.000 \mathrm{E}-01 \\
4.000 \mathrm{E}-01\end{array}$ & $\begin{array}{l}5.373 E-02 \\
5.255 E-02 \\
4.894 E-02 \\
3.734 E-02 \\
2.448 E-02\end{array}$ & $\begin{array}{l}5.373 E-02 \\
5.255 E-02 \\
4.894 E-02 \\
3.734 E-02 \\
2.448 E-02\end{array}$ \\
\hline $\begin{array}{l}5.000 \mathrm{E}-01 \\
6.000 \mathrm{E}-01 \\
7.000 \mathrm{E}-01 \\
8.000 \mathrm{E}-01 \\
9.000 \mathrm{E}-0.1\end{array}$ & $\begin{array}{l}1.399 E-02 \\
7.023 E-03 \\
3.121 E-03 \\
1.236 E-03 \\
4.405 E-04\end{array}$ & $\begin{array}{l}1.399 \mathrm{E}-02 \\
7.023 \mathrm{E}-03 \\
3.121 \mathrm{E}-03 \\
1.236 \mathrm{E}-03 \\
4.405 \mathrm{E}-04\end{array}$ \\
\hline $\begin{array}{l}1.000 \mathrm{E}+00 \\
1.500 \mathrm{E}+00 \\
2.000 \mathrm{E}+(10) \\
3.000 \mathrm{E}+00 \\
4.000 \mathrm{E}+00)\end{array}$ & $\begin{array}{l}1.451 E-04 \\
1.007 \mathrm{E}-0.5 \\
9.984 \mathrm{E}-06 \\
9.984 \mathrm{E}-06 \\
9.984 \mathrm{E}-06\end{array}$ & $\begin{array}{l}1.451 E-0.4 \\
1.007 E-05 \\
9.984 E-06 \\
9.984 E-06 \\
9.984 E-06\end{array}$ \\
\hline $\begin{array}{l}5.000 \mathrm{E}+00 \\
6.000 \mathrm{E}+00 \\
7.000 \mathrm{E}+00 \\
8.000 \mathrm{E}+00 \\
9.000 \mathrm{E}+00\end{array}$ & $\begin{array}{l}9.984 \mathrm{E}-06 \\
9.984 \mathrm{E}-06 \\
9.984 \mathrm{E}-06 \\
9.984 \mathrm{E}-06 \\
9.984 \mathrm{E}-06\end{array}$ & $\begin{array}{l}9.984 E-06 \\
9.984 E-06 \\
9.984 E-06 \\
9.984 E-06 \\
9.984 E-06\end{array}$ \\
\hline $1.000 \mathrm{E}+01$ & $9.984 E-06$ & $9.984 E-06$ \\
\hline
\end{tabular}

" Based on Stchest, 1970 
'T'ublo 2.30, Caso 4 Rosults: Concontrution of Np.237 in tho Rosk Matrix, at

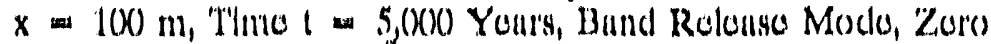
Disporsion, $D=1(0) \mathrm{m}^{2} / \mathrm{yr}$

\begin{tabular}{|c|c|c|}
\hline $\begin{array}{l}\text { Distance } \\
\mathrm{x}(\mathrm{m})\end{array}$ & FRACVAL & STENU* \\
\hline 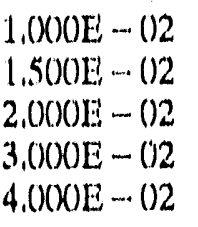 & $\begin{array}{l}4,361 \mathrm{E}-\mathrm{O} 2 \\
4,423 \mathrm{E}-\cdots(02 \\
4,482 \mathrm{E}-(12 \\
4,590 \mathrm{E}-(12 \\
4,(687 \mathrm{E}-\cdots(1)\end{array}$ & 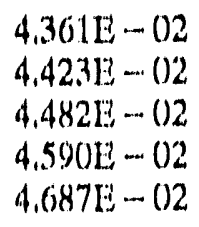 \\
\hline $\begin{array}{l}5.000 \mathrm{E}-02 \\
0.000 \mathrm{E}-02 \\
7.000 \mathrm{E}-02 \\
8.000 \mathrm{E}-02 \\
9.000 \mathrm{E}-02\end{array}$ & $\begin{array}{l}4,772 \mathrm{E}-(12 \\
4,845 \mathrm{E}-\ldots-(12 \\
4,90.5 \mathrm{E}-02 \\
4,955 \mathrm{E}-(12 \\
4,992 \mathrm{E}-(12\end{array}$ & $\begin{array}{l}4,772 \mathrm{E}-(122 \\
4,845 \mathrm{E}-(12 \\
4,9(05 \mathrm{E}-(12 \\
4,955 \mathrm{E}-(12 \\
4,992 \mathrm{E}-(12\end{array}$ \\
\hline $\begin{array}{l}1.000 \mathrm{E}-\ldots .01 \\
1.500 \mathrm{E}-0.1 \\
2.0(0) \mathrm{E}-01 \\
3.000 \mathrm{E}-0.01 \\
4.0(0) \mathrm{E}-01\end{array}$ & $\begin{array}{l}5 .(018 E-\cdots 02 \\
4.987 E-\ldots 2 \\
4.722 E-02 \\
3.735 E-02 \\
2.547 E-02\end{array}$ & $\begin{array}{l}5.018 \mathrm{E}-02 \\
4.987 \mathrm{E}-02 \\
4.722 \mathrm{E}-02 \\
3.735 \mathrm{E}-02 \\
2.547 \mathrm{E}-02\end{array}$ \\
\hline $\begin{array}{l}5.000 \mathrm{E}-01 \\
6.000 \mathrm{E}-01 \\
7.000 \mathrm{E}-01 \\
8.000 \mathrm{E}-01 \\
9.000 \mathrm{E}-01\end{array}$ & $\begin{array}{l}1.519 E-02 \\
7.982 E-0.3 \\
3.722 E-03 \\
1.549 E-03 \\
5.797 E-04\end{array}$ & $\begin{array}{l}1.519 \mathrm{E}-02 \\
7.982 \mathrm{E}-0.3 \\
3.722 \mathrm{E}-03 \\
1.549 \mathrm{E}-0.3 \\
5.797 \mathrm{E}-04\end{array}$ \\
\hline $\begin{array}{l}1.000 \mathrm{E}+00 \\
1.500 \mathrm{E}+00 \\
2.000 \mathrm{E}+00 \\
3 .(000 \mathrm{D}+00 \\
4.000 \mathrm{E}+00\end{array}$ & $\begin{array}{l}1.989 \mathrm{E}-04 \\
1.014 \mathrm{E}-0.5 \\
9.984 \mathrm{E}-06 \\
9.984 \mathrm{E}-06 \\
9.984 \mathrm{E}-06\end{array}$ & $\begin{array}{l}1.989 \mathrm{E}-04 \\
1.014 \mathrm{E}-0.5 \\
9.984 \mathrm{E}-06 \\
9.984 \mathrm{E}-06 \\
9.984 \mathrm{E}-06\end{array}$ \\
\hline $\begin{array}{l}5.000 \mathrm{E}+000 \\
6.000 \mathrm{E}+00 \\
7.000 \mathrm{E}+00 \\
8.000 \mathrm{E}+00 \\
9.000 \mathrm{E}+000\end{array}$ & $\begin{array}{l}9.984 E-06 \\
9.984 E-06 \\
9.984 E-06 \\
9.984 E-06 \\
9.984 E-06\end{array}$ & $\begin{array}{l}9,984 \mathrm{E}-06 \\
9.984 \mathrm{E}-06 \\
0,984 \mathrm{E}-06 \\
9.984 \mathrm{E}-06 \\
9.984 \mathrm{E}-06\end{array}$ \\
\hline $1.000 \mathrm{~L}+01$ & $9.984 \mathrm{E}-06$ & $9.984 E-06$ \\
\hline
\end{tabular}

- Based on Stefhest, 1970 
T'ublo 2.31. Case 4 Results: Concontration of Np.237 in tho Rock Mutrix, at $x=100 \mathrm{~m}$. Times $t=5,000$ Yours, Band Roleaso Modo, Zoro Dlspersion, $D=1,000 \mathrm{~m}^{2} / \mathrm{yr}$

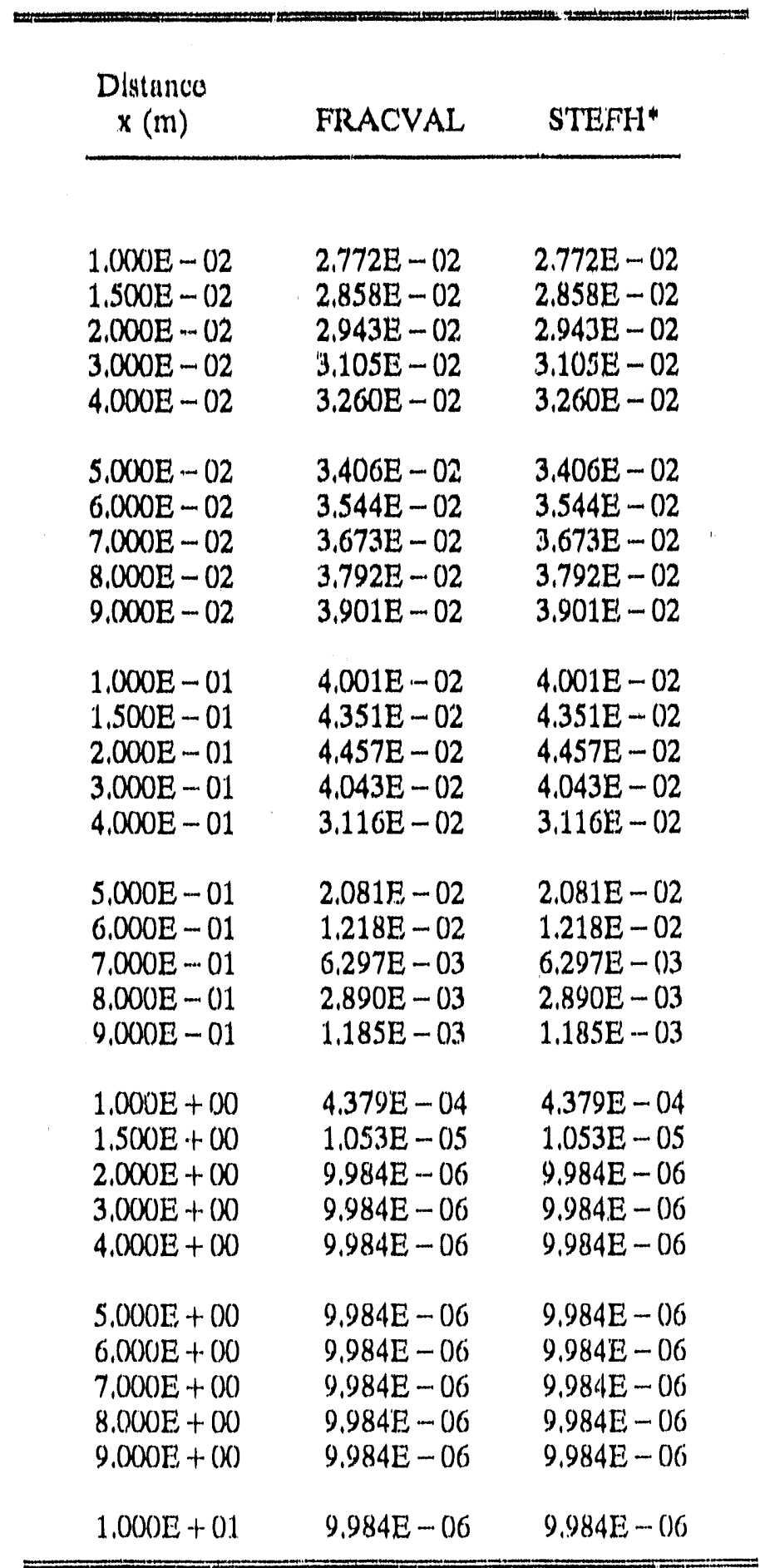

* Based on Stefhest, 1970 
Tublo 2.32, Cuso 4 Results: Concentration of Np-237 in the Rock Matrix, at $x=100 \mathrm{~m}$, T'lno $t$ a 5,000 Yoars, Band Reloaso Moclo, Zor's Dlsparsion, $D=10,000 \mathrm{~m}^{2} / \mathrm{yr}$

\begin{tabular}{|c|c|c|}
\hline $\begin{array}{l}\text { Distunce } \\
\mathrm{x}(\mathrm{m})\end{array}$ & FRACVAL & S'TEFH' \\
\hline 1.000$) E-(02$ & $1.282 \mathrm{E}-02$ & $1.282 \mathrm{E} \cdots 02$ \\
\hline $1.500 \mathrm{E}-02$ & $1.402 \mathrm{E}-02$ & $1.402 \mathrm{E}-02$ \\
\hline $2.000 \mathrm{E}-02$ & $1.521 \mathrm{E}-02$ & $1.521 \mathrm{E}-02$ \\
\hline $3.000 \mathrm{E}-02$ & $1.756 \mathrm{E}-02$ & $1.756 \mathrm{E}, \cdots 02$ \\
\hline $4 .(0)() E-1) 2$ & $1.986 \mathrm{E}-02$ & $1.986 \mathrm{E}-02$ \\
\hline $5.000 \mathrm{E}-02$ & $2.210 E-02$ & $2.210 \mathrm{E}-02$ \\
\hline $6.0000 \mathrm{E}-02$ & $2.428 \mathrm{E}-02$ & $2.428 \mathrm{E}-02$ \\
\hline $7.0000-02$ & $2.639 E-02$ & $2.639 \mathrm{E}-02$ \\
\hline $8.000 E-02$ & $2.842 \mathrm{E}-02$ & $2.842 \mathrm{E}-02$ \\
\hline $9.010 \mathrm{E}-02$ & $3,037 \mathrm{E}-02$ & $3.037 \mathrm{E}-02$ \\
\hline $1.000 \mathrm{E}-01$ & $3.223 E-02$ & $3.223 E-02$ \\
\hline $1.500 \mathrm{E}-01$ & $4.005 \mathrm{E}-02$ & $4.005 \mathrm{E}-02$ \\
\hline $2.000 E-01$ & $4.514 \mathrm{E}-02$ & $4.514 \mathrm{E}-02$ \\
\hline $3.000 \mathrm{E}-01$ & $4.699 E-02$ & $4.699 \mathrm{E}-02$ \\
\hline $4.000 \mathrm{E}-01$ & $4.009 \mathrm{E}-02$ & $4.009 \mathrm{E}-02$ \\
\hline $5.000 \mathrm{E}-0.1$ & $2.910 \mathrm{E}-02$ & $2.910 \mathrm{E}-02$ \\
\hline $6.000 E-01$ & $1.830 \mathrm{E}-02$ & $1.830 \mathrm{E}-02$ \\
\hline $7.000 E-01$ & $1.008 E-02$ & $1.008 \mathrm{E}-02$ \\
\hline $8.000 \mathrm{E}_{-}-01$ & $4.902 \mathrm{E}-03$ & $4.902 E-03$ \\
\hline $9.000 \mathrm{E}-01$ & $2.11 \mathrm{SE}-0.3$ & $2.116 \mathrm{E}-03$ \\
\hline $1.000 \mathrm{E}+00$ & $8.168 E-04$ & $8.168 \mathrm{E}-04$ \\
\hline $1.500 \mathrm{E}+00$ & $1.126 E-05$ & $1.126 \mathrm{E}-05$ \\
\hline $2,000 \mathrm{E}+00$ & $9.984 E-06$ & $9.984 E-06$ \\
\hline $3.000 \mathrm{E}+00$ & $9.984 \mathrm{E}-06$ & $9.984 E-06$ \\
\hline $4.000 \mathrm{E}+100$ & $9.984 \mathrm{E}-06$ & $9.984 E-06$ \\
\hline $5.000 \mathrm{E}+00$ & $0.984 E-06$ & $9.984 \mathrm{E}-06$ \\
\hline $6.000 \mathrm{E}+00$ & $9.984 \mathrm{E}-06$ & $9.984 \mathrm{E}-06$ \\
\hline $7,000 \mathrm{E}+00$ & $9.984 \mathrm{E}-06$ & $9.984 \mathrm{E}-116$ \\
\hline $8.000 E+00$ & $9.984 E-06$ & $9.984 E-06$ \\
\hline $9.000 \mathrm{E}+00$ & $9.984 E-06$ & $9.984 \mathrm{E}-06$ \\
\hline $1.000 \mathrm{E}+01$ & $9.984 \mathrm{E}-06$ & $9.984 \mathrm{E}-06$ \\
\hline
\end{tabular}

* Based on Stefhest, 1970 


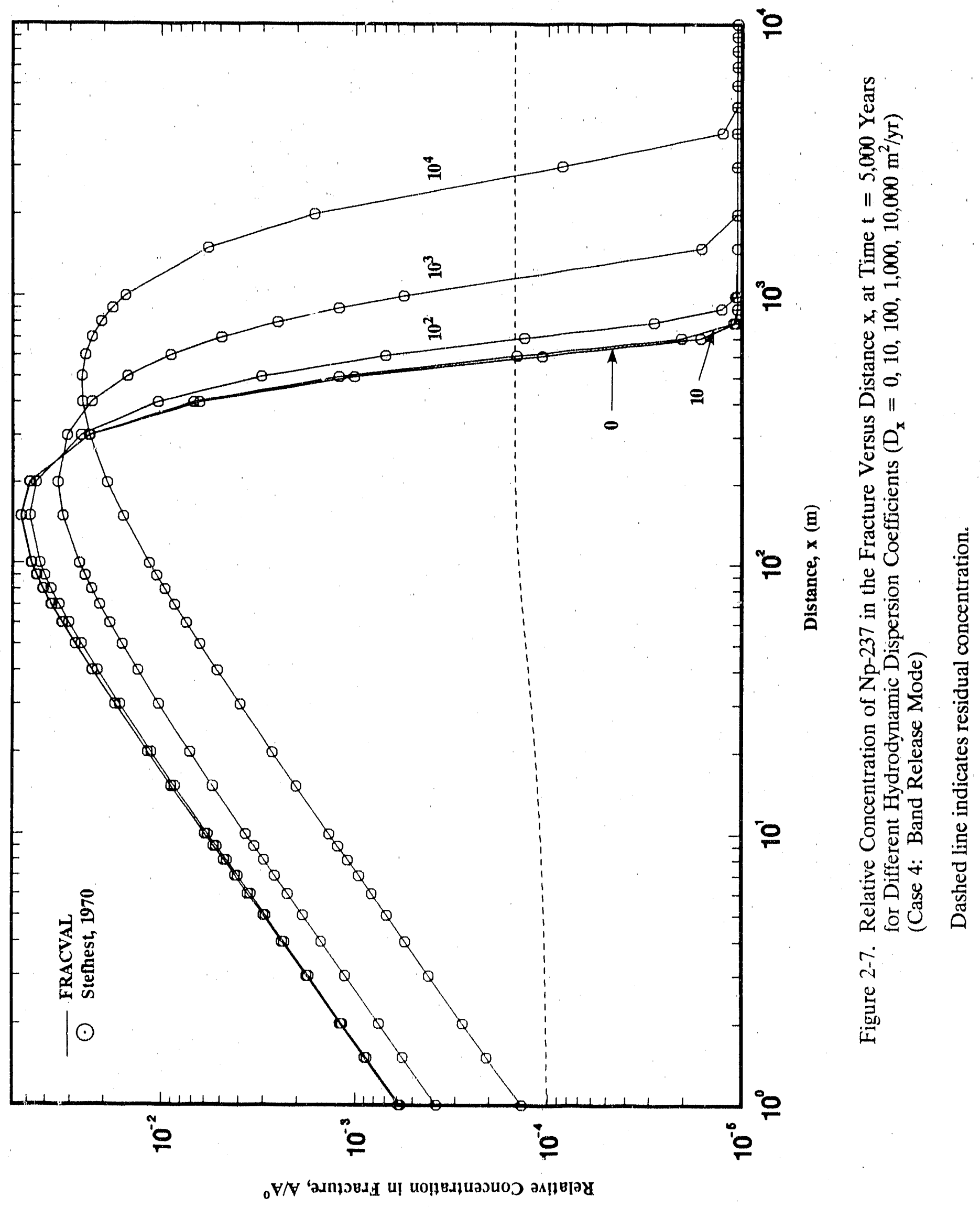




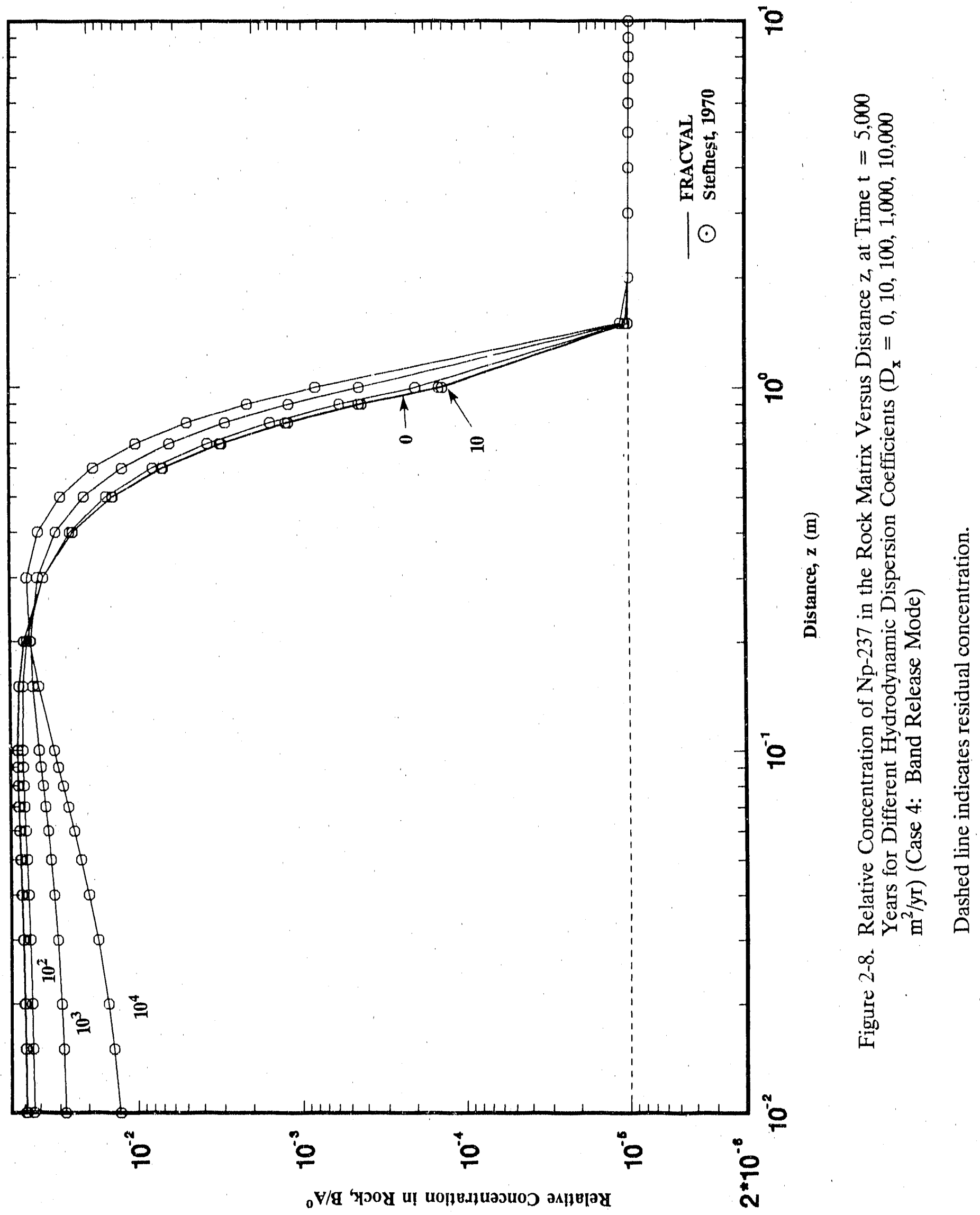




\subsection{SUMMARY AND CONCLUSIONS}

Analytical solutions based on the Laplace transforms have been derived for predicting the onodimensional, transient, advective-dispersive transport of a reacting radionuclide in a discrote planar fracture of constant aperture with infinite rock matrix diffusion. The particular features of these solutions reside in their additional analytical capability designed to handle residual concentrations in both fracture and rock matrix. The latter are represented by a constant and/or a spatially dependent, function in the case of the fracture and a constant in the case of the rock matrix. The closed-form solutions for the case of zero-dispersion have also been presented.

The reported solutions can handle an exponentially decaying source of solute at the upstream end of the fracture, which may be subject either to a step or band release mode. A convergent integration of the solution for the general case was obtained by means of a composite Gauss-Legendre quadrature scheme.

The module of the computer code FRACVAL which evaluates the analytical solutions presented in this report has been successfully tested on the one hand against works published by Van Genuchten and Alves (1982) and Gureghian (1990) dealing with particular aspects of the problem at hand, and by means of a solution based on a numerical inversion of the Laplace transforms proposed by Stefhest (1970) on the other hand.

The solutions presented in this section of the report are primarily related to performance assessment investigations of potential nuclear waste repository sites restricted to typical scenario analyses associated with long-term migration of radionuclides in an idealized fractured rock system. In addition, these solutions can be conveniently used to simulate the behavior of a decaying species in frac. tured rock within a laboratory environment and subsequently through a validation process to provide the investigator with site-specific information regarding the magnitude and importance of the various geochemical parameters gearing the transport process through the fractured rock system of interest. 


\subsection{REFERENCES FOR PAR'I' 1}

Bear, J., 1972. Dynamics of Fluid in Porous Media, American Kilsevior Publishing Co., Now York, NY.

Gradshteyn, I. S., and I. M. Ryzhik, 1980. Table of Integrals, Series and Products, Academic Press, Now York, NY, p. 1160.

Gureghian, A. B., 1990. FRACFLO: Analytical Solutions for 'Two I) Dincensional Iransport of a Decuying Species in a Discrete Planar Fracture and Equidistant Multiple Fiactures with Rock Matrix Diffusion, BMI/OWTD-5, ()ffice of Waste Technology Development, Battelle, Willowbrook, Il..

Gureghian, A. B., 1990. "STEFH: A Solution Based on the Numerical Inversion of the Laplace 'Trans. form for the One-Dimensional 'Transport of a Decaying Species in a Single Fracture with Rock Matrix Diffusion," Draft Cornputer Code. Available from project office.

Gureghian, A.B., and S. Steidl, 1990. FRACVAL User's Guideand XYPL,O'I Vorsion L fo for FRACVAL: Validation (Nonlinear Least Squares Method) of tho Solution of One D) imensional Transport of a Decaying Species in a Discrete Planar Fracture With Rock Matrix Diffusion, Battelle Office of Waste Technology Development, Willowbrook, IL.

Neretnieks, 1., 1980. "Diffusion in the Rock Matrix: An Important Factor in Radionuclide Retardation?" Journal of Geophysical Research, Vol. 85, pp. 4379-4397.

Neretnieks, I., 'T'. Eriksen, and P. 'T'ahtinen, 1982. "'Tracer Movement in a Single Fissue in Cranitic Rock: Some Experimental Results and Their Interpretation," Water Resourcos Rescarch, Vol. 18, No. 4, pp. 849-858.

Stefhest, H., 1970. "Numerical Inversion of laplace 'Iransforms," Commun. ACM, Vol. 13, No. 1, pp. $47-49$.

van Genuchten, M. Th., and W. J. Alves, 1982. Analytical Solutions of the One-I)imensional Convective-Dispersive Solute Transport Equations, U.S. Department of Agrictilture, Technical Bulletin No. 1661. 


\section{APPENIDIXA}

THEOREMS AND LAPLACL TRANSFORMS

In this appendix, a selected number of thooroms, inverse Laplaco transforms, and derivatives are reported (Abramowitz and Stegun, 1972).

\section{A.1 THEOREMS}

The operations for the Laplace transformation reported in Part 1 require, in some cases, the use of the following theorems.

\section{A.1.1 Translation}

$$
L^{-1}\left|e^{-b s} f(s)\right|=F^{\prime}(t-b) U(t-b), b>0
$$

where $U(t)$ is the Heaviside unit step function delined as

$$
U(t)=\begin{aligned}
& 0, t<0 \\
& 1, t>0
\end{aligned}
$$

\section{A.1.2 Linear Transformation}

$$
\text { L. }^{\cdots 1}|f(s-a)|=e^{\text {at }} F(t)
$$

\section{A.1.3 Differentiation}

$$
\begin{gathered}
L^{-1} s f(s)-F^{\prime}(+0)=F^{\prime}(t) \\
L^{-1} s^{n} f(s)-L^{-1} s^{n-1} F^{n}(+0)-L^{,-1} s^{(1-2} F^{\prime}(+0)-\ldots-F^{(n-1)}(+0)=F^{(n)}(t)
\end{gathered}
$$




$$
\begin{aligned}
& \frac{e^{-a \sqrt{s}}}{s} \quad=\operatorname{arfc}\left(\frac{a}{2 \sqrt{t}}\right) \\
& \frac{e^{-a \sqrt{s}}}{\sqrt{s}} \quad=-\frac{1}{\sqrt{\text { IIt }}} \exp \left(-\frac{a^{2}}{4 t}\right) \\
& e^{-a \sqrt{s}} \quad=\frac{a}{2 \sqrt{n t}^{3}} \exp \left(-\frac{a^{2}}{4 t}\right) \\
& \frac{1}{\sqrt{s(a+\sqrt{s})}} . \quad=e^{a^{2} t} \operatorname{erfc} a \sqrt{t} \\
& \frac{e^{-k \sqrt{s}}}{\sqrt{s(a+\sqrt{s})}}(k \geq 0) \quad=e^{a k} e^{a^{2} t} \operatorname{erc}\left(a \sqrt{t}+\frac{k}{2 \sqrt{t}}\right) \\
& \frac{1}{\sqrt{\mathrm{s}}+\mathrm{a}} \quad=\frac{1}{\sqrt{\text { IIt }}}-a e^{a^{2} t} \operatorname{erfc} a \sqrt{t} \\
& \frac{e^{-k \sqrt{s}}}{a+\sqrt{s}}(k \geq 0) \quad=\frac{1}{\sqrt{\text { IIt }}} \exp \left(-\frac{k^{2}}{4 t}\right)-a e^{a k} e^{a^{2} t} \operatorname{erfc}\left(a \sqrt{t}+\frac{k}{2 \sqrt{t}}\right)(\text { A.2-7) } \\
& \frac{e^{-k \sqrt{s}}}{s(a+\sqrt{s})} \quad=\frac{1}{a}\left|\operatorname{erfc} \frac{k}{2 \sqrt{t}}-e^{a k} e^{a^{2} t} \cdot \operatorname{erfc}\left(a \sqrt{t}+\frac{k}{2 \sqrt{t}}\right)\right| \\
& \frac{1}{s(a+\sqrt{s})}=\frac{1}{a}\left|1-e^{a^{2} t} \operatorname{erfc}(a \sqrt{t})\right|
\end{aligned}
$$

\section{A.3 APPENDIX A REFERENCE}

Abramowitz, M., and I. A. Stegun, 1972. Handbook of Mathematical Functions, Dover Publications, Inc., New York, NY. 


\section{APPENDIX B}

\section{QUADRA'TURE}

The solution presented in this report requires for the most part the evaluation of integrals. An exception is made in the case of the dispersion-free solution. The integrals associated with the general solution of Equations 2-30 and 2-34 with limits $(0, \infty)$ are evaluated by means of the Gauss-Legendre quadrature scheme (see Carnahan et al., 1969; Stroud and Secrest, 1966) after transformation of the original limits to linite ones.

\section{B.1 TRANSFORMATION FOR INFINI'IE IN'TEGRALSS ASSOCIATED WITH THE PRESENCE OF LONGITUDINAL DISPERSION}

The integral of interest has the following form

$$
I=\int_{0}^{\infty} f(x) d x
$$

when the dependent variable of the integrand corresponds in our case to o (see Equation 2-15). Hence by virtue of the properties inherent to the Heaviside function (see Equations 2-13), the lower limit, say $o_{\text {min }}$, musit meet the following criterion

$$
\mathrm{t} \geq \mathrm{RX}_{\mathrm{X}}
$$

Substitution for $\mathrm{x}$ given by Equation $229 \mathrm{~b}$. 'The potential lower limit may then be written as

$$
1^{\sigma} \min =\frac{x}{2}\left(\frac{R}{D_{x} t}\right)^{1 / 2}
$$

The choice of the upper and lower limits of integration are also dictated by the condition that the maximum absolute value of the exponential argument appearing in Equation 2-29a written as

$$
\Psi(x, \sigma)=\frac{2}{\sqrt{\pi}} \exp \left[-\left(\sigma-\frac{y}{\sigma}\right)^{2}\right]
$$

with

$$
Y=\frac{u x}{4 D_{x}}
$$

should always be less than or equal to the one allowed by the computer, say AR(x. Hence equating the latter with the argument of the exponential form in Equation B-4, we obtain

$$
\sigma^{4}-(A R G+2 y) \sigma^{2}+y^{2}=0
$$


where the roots of the above equation are given by

$$
\begin{aligned}
& \sigma_{\max }^{2}=\frac{(A R G+2 \gamma)+\left|(A R G+2 \gamma)^{2}-4 y^{2}\right|^{1 / 2}}{2} \\
& 2 \sigma_{\min }^{2}=\frac{(A R G+2 \gamma)-\left|(A R G+2 \gamma)^{2}-4 \gamma^{2}\right|^{1 / 2}}{2}
\end{aligned}
$$

Note that both roots are positive. The potential values for the integration limits given by Equa-

tions B-7 and B-8 are now used to extract suitable upper ( $\left.\sigma_{U L}\right)$ and lower limits $\left(\sigma_{\mathrm{LL}}\right)$. The integration domain is split over two finite intervals, $\mathrm{i}, \mathrm{D},\left[\sigma_{\mathrm{LL}}, \sigma_{I N}\right]$ and $\left[\sigma_{I N}, \sigma_{U L}\right]$, whero $\sigma_{I N}$ will correspond to the value of o in Equation B-6 when function $\psi(x, 0)$ (see Equation 2-29a) is a maximum:

$$
\sigma_{I N}=Y^{1 / 2}, \sigma_{L L}<Y^{1 / 2}<o_{U L}
$$

If the above condition is not satisfied, then

$$
\sigma_{I N}=\frac{1}{2}\left(\sigma_{L L}+\sigma_{U L}\right), Y^{1 / 2}<\sigma_{L L} \text { or } \gamma^{1 / 2}>\sigma_{U L}
$$

where

$$
\begin{aligned}
& \sigma_{L L}=\operatorname{MAX}\left[\sigma_{1} \sigma_{\min } 2^{\sigma_{\min }}\right] \\
& \sigma_{U L}=\operatorname{MAX} I_{1} \sigma_{\min }, \sigma_{\max } l
\end{aligned}
$$

Results yielded by this method proved to be very satisfactory, however, at the expense of "an additional computational effort.

\section{REFERENCES}

Carnahan, B., H. A. Luther, and J. O. Wilkes, 1969. Applied Numerical Methods, John Wiley and Sons, Inc., New York, NY.

Stroud, A. H., and D. Secrest, 1966. Gaussian Quadrature Formulas, Prentico-Hall, Inc, Englewood Cliffs, N.J. 


\section{APPENDIX C}

\section{EVALUATTON OF ERROR PUNCIION AND PRODUC'I OF'

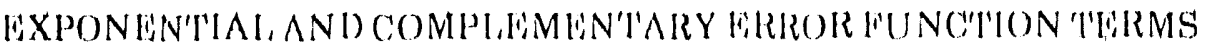

Tho error or probabillty function is definod as

$$
\operatorname{cor}(x)=\frac{2}{\sqrt{11}} \int_{0}^{x} e^{-\xi_{1}^{2}} d \xi
$$

with

$$
\operatorname{arf}^{\prime}(-x)=-\operatorname{arf}(x)
$$

When $\mathrm{x}$ is small the integrand may bo conveniently expanded in a power sories convorgent overywhore and integrated term by torm to yiold

$$
\begin{aligned}
\operatorname{arf}(x)=\frac{2}{\sqrt{\pi}} \mid x & -\frac{x^{3}}{3 \cdot 1 !}+\frac{x^{5}}{5 \cdot 2 !}-\frac{x^{7}}{7 \cdot 3 !}+\ldots \\
& +(-1)^{1 n+1} \frac{x^{2 n+1}}{(2 n+1) \cdot n !}+\ldots \mid
\end{aligned}
$$

A few terms in the expansion are necessary to dotormine tho valuo of erf $x$ to a given number of decimal places. However, as $\mathrm{x}$ becomes large the loss in accuracy must be cornpensatod by a large number of terms which ronders the calculation tedious and impractical. A rational Chohyshob approximation may be used to alleviate this problem when $x \geq 4$ (seo Cody, 1969). Alternatively, the asymp. totic expansion reported by Abramowitz and Stegun (1964) expressed in terms of the complemontary error function erfc ( $\mathrm{x}$ ) (Equation $\mathrm{C}$-1) may also be written as

$$
\operatorname{erf}(x)=1-\operatorname{erfc}(x)
$$

where

$$
\operatorname{arfc}(x)=\frac{2}{\sqrt{I I}} \int_{x}^{\infty} e^{-\xi^{2}} d \xi
$$

In vlew of Equations (C-2) and (C-4), we have

$$
\operatorname{arfc}(-x)=2-\operatorname{erfc}(x)
$$




\section{Asymplotic Expanylon}

The asymptotic uxpansion of orfe( $x$ ) as givon by Abramowita and Stegun (1964) may bo writton as

$$
\operatorname{arfc}(x)=\frac{0^{-x^{2}}}{x \sqrt{11}}\left|1+\sum_{n=1}^{\infty}(-1)^{n} \frac{1 \cdot 3 \ldots(2 n-1)}{\left(2 x^{2}\right)^{n}}\right|+R_{11}(x)
$$

If $R_{n}(x)$ is the romainder after $n$ torms then

$$
R_{n}(x)=(-1)^{n} \frac{1 \cdot 3 \ldots(2 n-1)}{\left(2 x^{2}\right)^{n}} \int_{0}^{(x)} 0^{-1}\left(1+\frac{t}{x^{2}}\right)^{-n-t} d t
$$

$R_{11}(x)$ is less in absolute value than the first noglected torm and of the same sign.

The solution of Equations (2-30) and (2-34) includes terms involving products of un exponential und complementary error functions, respectively. 'Tho ovaluation of such torms is performed by means of function EXP_ERFC in the FRACVAL mathematical modol describod bolow, and which uses the propertios of the error and complemontary error functions outlined above, latting a or $\beta$ correspond to the argument of tho exponontial and complomentary orror function, respectivoly, evaluato

$$
S=a-\beta^{2}
$$

using Eq. (C-7) and noglecting the remuinder term. 'The product of exp(a) and erfe( $(\beta)$ is givon by

$$
\omega a=\frac{\exp (s)}{\beta \sqrt{\pi}}\left|1+\sum_{n=1}^{\infty}(-1)^{n} \frac{1.3 \ldots(2 n-1)}{\left(2 \beta \beta^{2}\right)^{n}}\right|
$$

and with

$$
\mathrm{Vic}_{\mathrm{c}}=\exp (\mathrm{a}) \mathrm{erfc}(\beta)
$$

where erfe( $\beta$ ) is evaluatod based on Cody (1969), the calculation of EXP_ERFC proceods as outlined in Figure C-1. Note that EXMAX is the largest positivo argument of the exponential function allowed by a particular computer. 


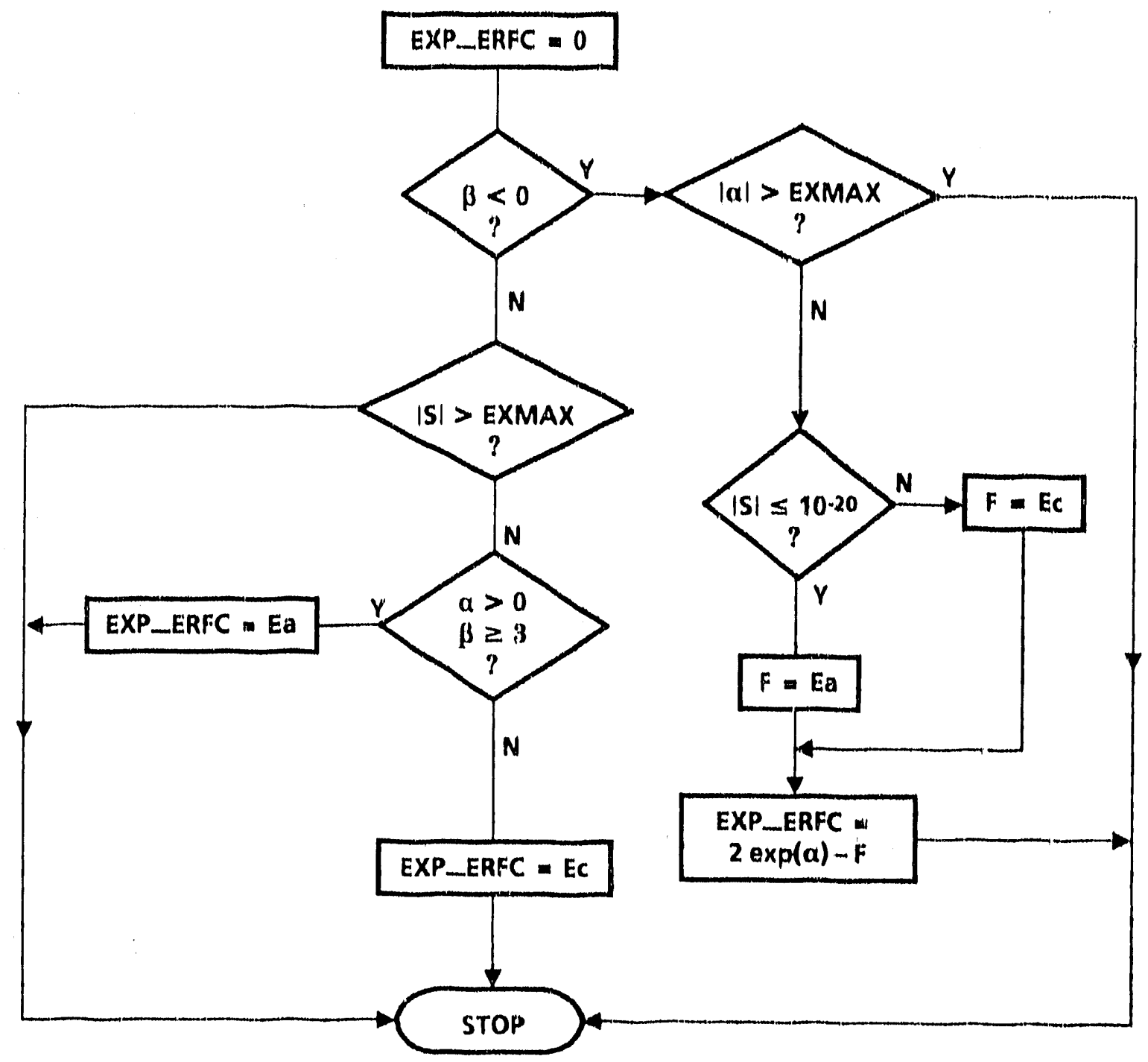

Figure C-1. Bvaluation of EXP_ERFC 


\section{Ruforoncess}

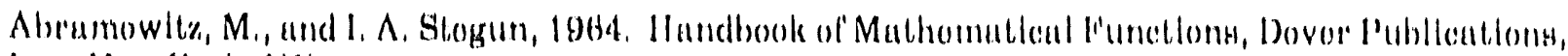
Inc, Now Yor'k, NY.

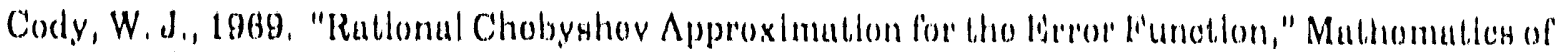
Computation, Vol, 23, No, 107, pp, 831.837. 


\section{APHINIIX D}

\section{NO'I'A'IITNS}

\begin{tabular}{|c|c|}
\hline$\|1\| 2, a$, & 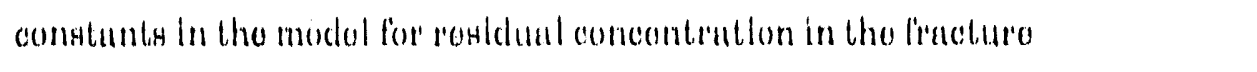 \\
\hline আ, & longlludlnal dlepourylvily, \\
\hline$\Lambda$ & oncontention in tho fracturo \\
\hline$\Lambda^{\prime \prime}$ & concontritlon of the spocies al, the soures at limo equals zaro \\
\hline b & half-thicknoss of tho frachuro \\
\hline$b_{1}$ & rosidual concontration in tho rock matrix \\
\hline 13 & concontration In thos rock matrix \\
\hline$D_{1}$ & molocular diffuslon of nuceledo in wator \\
\hline 1$)_{0}$ & offectlvo diffuston coodficiont in tho rock matrix \\
\hline$D_{\mu}$ & poro diffusivity \\
\hline 1$)_{x}$ & hydrodymumle dispersion oofffelont in tho fructuro \\
\hline$t_{i}$ & goomotric fuctor $\left(\delta_{1} / l^{\prime}, 2\right)$ \\
\hline J & diffusivo rato of nuclide at surfice of fracturo por unit arou of frecturo surface \\
\hline$K_{f^{\prime}}$ & surfaco distribution confliciont in tho fracture \\
\hline$K_{r}$ & distribution cooffictent in the rock matrix \\
\hline K & roturdation factor in tho fracturo \\
\hline $\mathrm{R}^{\prime}$ & rotardation fuctor in tho rock matrix \\
\hline $\mathrm{t}$ & $\operatorname{limo}$ \\
\hline $\mathrm{T}$ & leaching tline \\
\hline $\mathrm{T}_{1 / 2}$ & half-lifo \\
\hline 11 & avorago fluid volocily voctor in the fraoturo \\
\hline$x$ & posltion voctor in the fracture \\
\hline « & position vector in the rock matrix \\
\hline$\delta_{d}$ & constrictivity for diffusion \\
\hline
\end{tabular}


flrst-ordor rato constant for docay

$p_{n}$ rock donsily

t

tortuosity of tho fractures

$\iota^{\prime} \quad$ lortuosity of the rock malrix

$\Phi$

rook porosily 


\section{ÁPPENDIX E}

\section{MODEL,PARAMETERS}

ALFA Constant alpha in the exponential term in the residual concentration model in the fracture $(1 / \mathrm{L})$

CC0 Concentration of the species at the source at time equals zero (units of activity/L3)

CINF(1) Constant in residual concentration model in the fracture (units of activity/L,3)

CINF(2) Cinfficient of exponential term in residual concentration model in the fracture (units of activity/L3)

CINR Residual concentration in the rock matrix (units of activity/L $\mathrm{L}^{3}$ )

DENSR Rock matrix bulk density $\left(\mathrm{M} / \mathrm{L}^{3}\right)$ (used if IDIST $(2)=1$ )

DIFF Molecular diffusion of species in water $\left(\mathrm{L}^{2} / \mathrm{T}\right)$ (used if IDISP $=1$ )

DIFFR Pore diffusivity $\left(\mathrm{L}^{2} / \mathrm{T} / \mathrm{T}\right)(\mathrm{IGEOF}=0)$ or geometric factor $(\mathrm{L} / \mathrm{L})(\mathrm{IGEOF}=1)$

MWTNS(I,J) Dimensions used in the problem; each must be $\leq 12$ characters in length

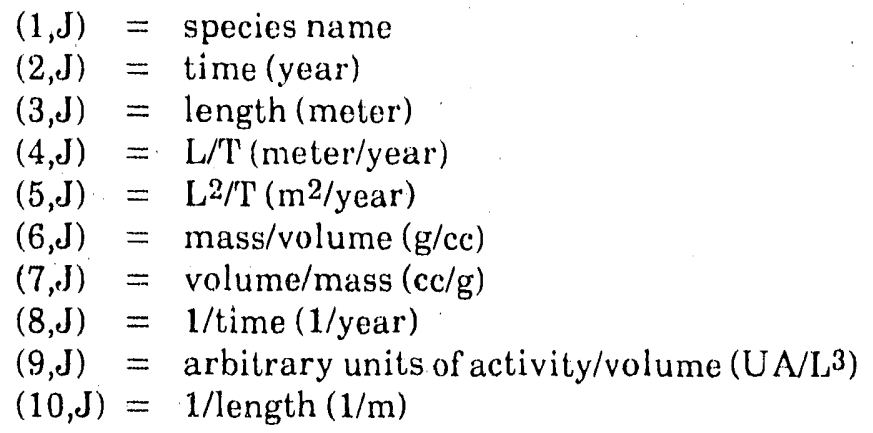

DISPX Hydrodynamic dispersion coefficient $\left(\mathrm{L}^{2} / \mathrm{T}\right)(\mathrm{IDISP}=0)$ or Longitudinal dispersivity $($ L) $($ IDISP $=1)$

DISTRB_F Fracture surface distribution coefficient (L) [IDIST $(1)=1]$

DISTRB_R Rock matrix distribution coefficient (L $3 / \mathrm{M})$ [IDIST $(2)=1]$

EXMAX* Largest allowed magnitude for exponential arguments

HALFL Half-life of species (T)

HALF_ THICK Half-thickness of the fracture (L)

* Computer-dependent. 
- 0 User supplies arrays REFX, REF', and 'TIME, including parameters $N X, N Z$, and $\mathrm{NT}$

$=1$ Automatic generation of arrays RFFX, REFZ, and TIME, including parameters $\mathrm{NX}, \mathrm{NZ}$, and $\mathrm{N}^{\prime} \mathrm{T}$ (see Note)

IBAND

$=0$ Step release mode at source

$=1$ Band release mode at source

ICONCF $\quad=0$ Do not calculate fracture concentrations

$=1$ Do calculate fracture concentrations

ICONCR

$=0$ Do not calculate rock concentrations

$=1$ Do calculate rock concentrations

IDISP

$=0$ DISPX, corresponds to dispersion coefficient in fracture

$=1$ DISPX, corresponds to dispersivity in fracture

IDIST(1) = $=0$ RETARD_F corresponds to retardation factor in fracture

$=1$ RETARD_F corresponds to surface distribution coefficient in fracture, i.e.,

DISTRB F

IDIST(2) $\quad=0$ RETARD_ $R$ corresponds to retardation factor in rock matrix

$=1$ RETARD_R corresponds to distribution coefficient in rock matrix, i.e.,

DISTRB_R

IGAUSL $\quad=0$ Interval of integration constant

$=1$ Interval of integration limits divided in INTERV/2 subintervals

IGEOF $\quad=0$ DIFFR corresponds to pore diffusivity

$=1$ DIFFR corresponds to geometric factor

IGRAPH $\quad=0$ Graphics output disabled

$=1$ Graphics output enabled; formatted graphics written to logical unit 30

INTERV $\quad$ Number of subintervals $\times 2$ for Gauss-Legendre integration (i.e:, $\leq 4$ (used if

IGAUSL $=1)$ )

MSG1

$\leq 80$ characters, first line of graphics output

MSG2

$\leq 80$ characters, second line of graphics output

NMAX Number of Gaussian points for Gauss-Legendre quadrature (can have only values of $4,10,20,60,104$, or 256$)$

NRUNMAX Number of data sets to be run

N'T

$\leq 500$, number of time values to be evaluated (skip if IAUTO $=1$ )

NVAL Index for selecting solution module

$=0$ Option for analytical solution

$=1$ Option for optimization module

NX

$\leq 500$, number of positions to be evaluated in $\mathrm{x}$ direction (skip if IAUTO $=0$ ) 
POROSR Average porosity in rock matrix $\left(\mathrm{L}, 3 / \mathrm{L}^{3}\right)$

REFX(I) $\quad x$-position in space (read if IAUTO $=0$ )

REFZ(I) $\quad$ ¿-position in space (read if IAUTO $=0$ )

RETARD_F Retardation factor IIDIST $(1)=01$ or surface distribution coefficient in the fracture, i.e., DISTRB_F IIDIST'(1) $=1]$

RETARD_R Retardation factor $\mid I D I S T(2)=0$ ] or distribution coefficient in the rock, i.e., DISTRB_R[IDIST $(2)=11$

TIME(I) t-position in time ('T) (read if IAUTO $=0$ )

TIML $\quad$ Leaching time (T) (used if IBAND $=1$ )

TITLE 2 lines, $\leq 80$ characters per line, title of data set

'TOR'T Tortuosity of the fracture (L") (used if IDISP $=1$ )

VELX Average fluid velocity in the fracture $(\mathrm{L} / / \mathrm{T})$

XDISP $\quad$ Longitudinal dispersivity (L) $($ IDISP $=1)$

Note: The following parameters are read in if IAUTO $=1$ in order to generate arrays REFX, REFZ, and TIME, and their associated parameters NX, NZ, and NT:

$\mathrm{X}$

First value of spatial coordinate $x=\operatorname{REFX}(1)$

DX

Spatial increment (skip if $N L O G=1$ )

ENDX

End value of spatial coordinate $x=\operatorname{REFX}(\mathrm{NX})$

Z0

First value of spatial coordinate $\mathrm{z}=\mathrm{REFZ}(1)$

DZ

Spatial increment (skip if NLOG = 1)

ENDZ

End value of spatial coordinate $\mathrm{z}=\operatorname{REFZ}(\mathrm{NZ})$

T0

First value of simulation time $t=\operatorname{TIME}(1)$

D'T

Time increment (skip if $N L O G=1$ )

END'T

Erid value of simulation time $=$ TIME(NZ)

NLOG $\quad=0$ Positions in space or time are equally spaced

$=1$ Log scale used for splitting space or time arrays (i.e., 10 divisions per log cycle) 

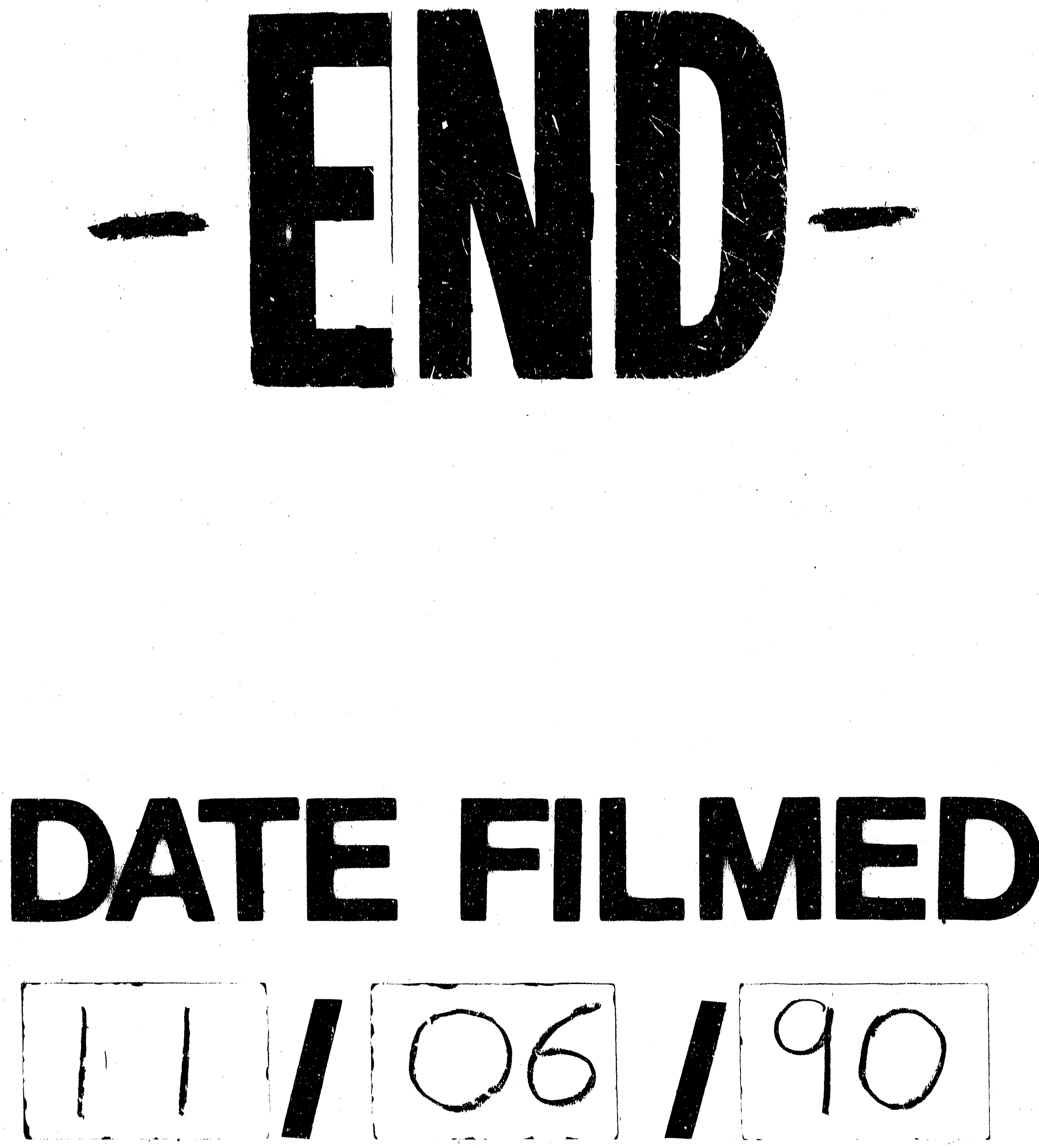
